Sofía Sánchez Orois

\title{
Untersuchungen zur optimalen Steuerung der Waldentwicklung
}

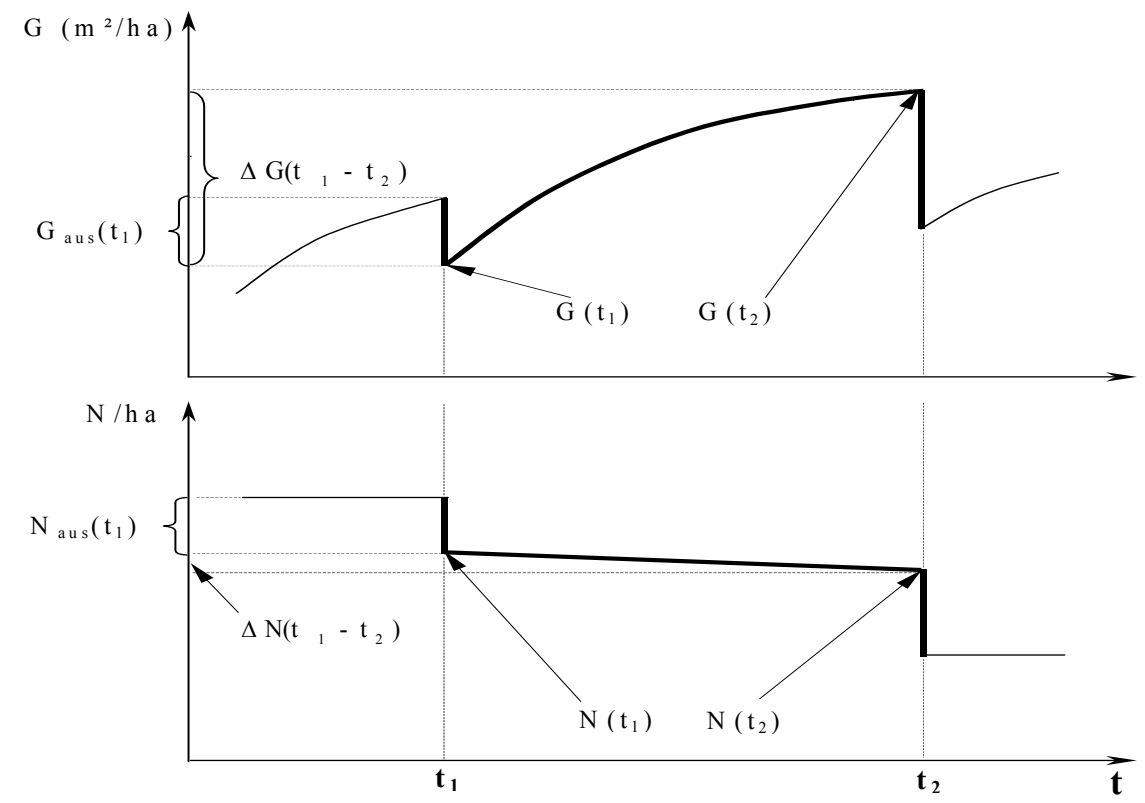





\title{
Untersuchungen zur optimalen Steuerung der Waldentwicklung
}

\author{
Dissertation zur Erlangung des Doktorgrades \\ der Fakultät für Forstwissenschaften und Waldökologie \\ der Georg-August-Universität Göttingen
}

\author{
vorgelegt von \\ Sofía Sánchez Orois \\ geboren in Arzúa (Spanien)
}

Göttingen, im Mai 2003 
D7

1. Berichterstatter:

Prof. Dr. Dr. h.c. Klaus von Gadow

2. Berichterstatter:

Prof. Dr. Roque Rodríguez Soalleiro

3. Berichterstatter:

Prof. Dr. Dr. h.c. Branislav Sloboda

Tag der mündlichen Prüfung: $\quad$ 27. Juni 2003

Diese Arbeit wurde durch die Niedersächsische Staats- und Universitätsbibliothek, SUBGöttingen, unter folgender Internetadresse veröffentlicht:

http://webdoc.sub.gwdg.de/diss/2003/sanchez_orois/ 


\section{Danksagung}

Die vorliegende Arbeit wurde am Institut für Waldinventur und Waldwachstum der GeorgAugust-Universität Göttingen verfasst. Meinem Doktorvater Prof. Dr. Klaus von Gadow danke ich nicht nur für die wissenschaftliche Anleitung sondern auch für die liebenswürdige Art und Weise der Unterstützung. Insbesondere schätze ich sein Vertrauen zu mir, die Förderung meiner Veröffentlichungen und meiner Vorträge auf wissenschaftlichen Tagungen. Meinem spanischen Betreuer Prof. Dr. Rodríguez Soalleiro danke ich für die intensive Betreuung während seines Aufenthaltes am göttinger Institut für Waldwinventur und Waldwachstum und für die kritischen Anmerkungen zu meinen Manuskripten. Ich möchte mich auch bei Prof. Dr. Branislav Sloboda für die Unterstützung und guten Vorschläge, die meine erste wissenschaftliche Publikation ermöglicht haben, sowie für die Bereitschaft als Gutachter zu fungieren bedanken.

Meinen Institutskollegen danke ich für die gute Arbeitsatmosphäre und die entspannenden Teepausen. Besonderer Dank gilt Herrn Dipl.-Ing. Fabian Röhnisch, Herrn Dr. Matthias Albert, Herrn M.Sc. Lars Hinrichs und Frau Rüdiger für die kritischen Anmerkungen und guten Vorschläge, die zur Verbesserung dieser Arbeit beigetragen haben.

Ganz herzlicher Dank gilt meinen Eltern für ihr Verständnis und jederzeitige Unterstützung.

Und weil man von Luft und Liebe nicht leben kann, danke ich den galicischen Stiftungen „Pedro Barrié de la Maza“ und „Caixa Galicia“ für die großzügige Förderung meines Aufenthaltes am Institut für Waldinventur und Waldwachstum. 



\section{Vorwort}

Die vorliegende Dissertation gliedert sich in fünf Manuskripte, die sich mit der Steuerung der Waldentwicklung befassen. Die Manuskripte sind mit der Genehmigung der Fakultät für Forstwissenschaften und Waldökologie der Georg-August Universität Göttingen in wissenschaftlichen Fachzeitschriften erschienen bzw. eingereicht. Die Problematik und die Lösungsansätze, vor allem die Methoden der Generierung von Bestandesentwicklungsszenarien unter Verwendung neuer Optimierungsverfahren werden am Beispiel von gleichaltrigen Fichten- und ungleichaltrigen Kiefernbeständen dargestellt. Aufgrund der methodischen Unterschiede lässt sich diese Arbeit in zwei Kapitel einteilen:

I. Untersuchung zur optimalen Steuerung der Waldentwicklung am Beispiel von gleichaltrigen Fichtenbeständen in Deutschland

II. Untersuchung zur optimalen Steuerung der Waldentwicklung am Beispiel von ungleichaltrigen Kiefernbeständen in Galicien.

Das erste Kapitel (Manuskripte I-III) befasst sich mit der Modellierung des Wachstums und schließlich mit der Optimierung von Vornutzungen in gleichaltrigen Fichtenreinbeständen.

In dem zweiten Kapitel (Manuskripte IV-V) wird die Modellierung des Wachstums in Kiefern-Laubwäldern (überwiegend mit Eiche) in Galicien (Nordwest Spanien) behandelt. Anschließend wird eine Untersuchung zur Bestimmung des optimalen Vorrats und Nutzungszyklus für das ungleichaltrige Management beschrieben sowie ein Vergleich zwischen dem Kahlschlagsystem (in Englisch als Rotation Forest Management bekannt) und dem kahlschlagfreien, selektiven Nutzungssystem (in Englisch als Continuous Cover Forestry System benannt) durchgeführt.

Die Manuskripte sind in folgenden referierten, wissenschaftlichen Zeitschriften erschienen bzw. eingereicht.

I. Gurjanov, M., Sánchez Orois, S. und Schröder, J. 2000: Grundflächenmodelle für gleichaltrige Fichtenreinbestände. Eine vergleichende Analyse. Centralblatt für das gesamte Forstwesen 3/4:187-198.

II. Sánchez-Orois, S., Gurjanov, M. und Schröder, J. 2001: Analyse des Grundflächenzuwachses gleichaltriger Fichtenreinbestände. Allgemeine Forst- und Jagdzeitung 3:51-60. 
III. Sánchez Orois, S. und Vilčko, F. 2002: Bewertung und Optimierung von Vornutzungen in Fichtenbeständen. Forstwissenschaftliches Centralblatt 121:250264.

IV. Sánchez Orois, S. and Rodríguez Soalleiro, R. 2002: Modelling the growth and management of mixed uneven-aged maritime pine-broadleaved species forest in Galicia northwestern Spain. Scandinavian Journal of Forest Research 17(6): 537-547.

V. Sánchez Orois, S., Chang, J. S. and Gadow, K. v. 2002: Optimal residual growing stock and cutting cycle in mixed uneven-aged maritime pine stands in northwestern Spain. Forest Policy and Economics (im Druck). 


\section{INHALTSVERZEICHNIS}

1. Die STEUERUng DER WALDENTWICKLUNG: THEORETISCHE GRUNDLAGEN

2. UNTERSUCHUNG ZUR OPTIMALEN STEUERUNG DER WALDENTWICKLUNG AM BEISPIEL VON GLEICHALTRIGEN FICHTENBESTÄNDEN

2.1. EINLEITUNG

2.1.1. Die Fichte in Deutschland: bisherige Untersuchungen

2.1.2. Wuchsmodelle und ihre Anwendung

2.2. MODELLIERUNG DES GRUNDFLÄCHENWACHSTUMS BEI DER FICHTE

2.3. MOdelLANWENDUNG: BEWERTUNG UND OptimiERUNG VON BEHANDLUNGSOPTIONEN

3. UNTERSUCHUNG ZUR OPTIMALEN STEUERUNG DER WALDENTWICKLUNG AM BEISPIEL VON UNGLEICHALTRIGEN KIEFERNBESTÄNDEN IN GALICIEN

3.1. EINLEITUNG

3.1.1. Pinus pinaster Ait: Ihre Bedeutung für die Forstwirtschaft Galiciens

3.1.2. Bisherige Untersuchungen zum Wachstum der Pinus pinaster in Galicien

3.2. MODELLIERUNG DES WACHSTUMS UNGLEICHALTRIGER KIEFERNBESTÄNDE

3.3. MODELLANWENDUNG: BEWERTUNG UND OPTIMIERUNG VON BEHANDLUNGSOPTIONEN 15

4. DISKUSSION

4.1. ANWENDUNG DER ENTWICKELTEN MODELLE.

4.2. GENERIERUNG UND OPTIMIERUNG VON MANAGEMENTOPTIONEN

5. ZUSAMMENFASSUNG

\section{VERÖFFENTLICHUNGEN:}

GURJANOV, M., SÁNCHEZ OROIS, S. UND SCHRÖDER, J.

GRUNDFLÄCHENMODELLE FÜR GLEICHALTRIGE FICHTENREINBESTÄNDE

SANCHEZ Orois, S., GuRJANOV, M., UND SCHRÖDER, J.

ANALYSE DES GRUNDFLÄCHENZUWACHSES GLEICHALTRIGER FICHTENREINBESTÄNDE

SÁNCHEZ OROIS, S. UND VILČKO, F.

BEWERTUNG UND OPTIMIERUNG VON VORNUTZUNGEN IN FICHTENBESTÄNDEN

SÁNCHEZ OROIS, S. UND RODRÍGUEZ SOALLEIRO, R.

MODELLING THE GROWTH AND MANAGEMENT OF MIXED UNEVEN-AGED MARITIME PINEBROADLEAVED SPECIES FOREST IN GALICIA NW SPAIN

SÁNCHEz Orois, S., CHANG, S. J. UND GADOW, K.v.

OPTIMAL RESIDUAL GROWING STOCK AND CUTTING CYCLE IN MIXED UNEVEN-AGED MARITIME PINE STANDS IN NORTHWESTERN SPAIN 



\section{Die Steuerung der Waldentwicklung: Theoretische Grundlagen}

$\mathrm{Zu}$ den Aufgaben der Wissenschaftsdisziplin Forsteinrichtung (engl. Forest Management) gehört die Zustandserfassung, die Zustandsbeschreibung und die Steuerung der Waldentwicklung unter Berücksichtigung vielfältiger Ansprüche und Beschränkungen. Dabei wird gewöhnlich unterschieden zwischen der Bestimmung von Maßnahmen für einzelne Bestände und der Abstimmung der Einzelmaßnahmen mit der gesamtbetrieblichen Entwicklung. Eine wichtige Aufgabe der Forsteinrichtung besteht darin, die große Vielfalt der waldbaulichen Möglichkeiten zu erkennen. Diese Kenntnis wird genutzt, um die nachhaltige Waldentwicklung zu gewährleisten (Gadow, 2001).

Das klassische Ertragstafelsystem kennt nur eine Option der Waldentwicklung, nämlich die Vorgaben der Ertragstafel, nach denen die Stärke und Art der Eingriffe festgelegt sind. Wegen der Änderung der forstpolitischen Rahmenbedingungen und der waldbaulichen Ziele in den letzten Jahren stellen sich neue Aufgaben für die Forstplanung. Der Wald wird heute nicht nur als Produktionsstätte des Rohstoffes Holz sondern auch als multifunktionales Ökosystem betrachtet. Wegen der Vielfalt der Einzelentscheidungen verliert das einfache Prinzip der Normalvorgaben seine Gültigkeit. Deshalb wurden Methoden entwickelt, mit deren Hilfe Managementoptionen für unterschiedliche Entscheidungsebenen bewertet werden können.

Ziel der vorliegenden Arbeit ist die Generierung, Bewertung und anschließend die Optimierung von Managementoptionen am Beispiel von gleichaltrigen Fichtenbeständen und ungleichaltrigen Kiefernbeständen.

Ein Forstbetrieb, bzw. eine Waldlandschaft, umfasst eine diskrete Anzahl von lagemäßig definierten Beständen oder eine diskrete Anzahl von Straten. Die Straten bzw. Bestandestypen umfassen definitionsgemäß einzelne Bestände, die bestimmte ähnliche Merkmalskombinationen aufweisen, z.B. gleiche Baumartenanteile, Durchmesserverteilungen und Grundflächen.

Mit Hilfe der Methoden der Unternehmensforschung lässt sich die Planung für einzelne Straten relativ einfach gestalten. In diesem „Modell der Stratenoptionen“ werden die Entscheidungsvariablen durch den Vektor $\mathbf{x}$ verkörpert. Das Element $x_{i j}$ stellt die Fläche im Stratum $i$ des Betriebes (bzw. der Waldlandschaft), dem die Option $j$ zugeordnet wird, dar. Jede Option ist durch eine spezielle Abfolge natürlicher Bestandesentwicklungen und forstlicher Eingriffe gekennzeichnet. Jede Option $j$ im Bestandestyp $i$ hat einen den 
übergeordneten Zielsetzungen entsprechenden Nutzen $c_{i j}$ pro Flächeneinheit, z.B. den Vermögenswert, die Naturnähe oder die Diversität. Der Gesamtnutzen soll bei zielgerichtetem Verhalten möglichst hoch sein.

Eine Beschränkung ergibt sich dadurch, dass die Summe der Flächen der Optionen im Stratum $i$ genau gleich der Stratenfläche $a_{i}$ sein muss. Diese Beschränkung wird durch den Vektor a dargestellt. Zusätzlich können gesamtbetriebliche Beschränkungen erforderlich sein. Der Vektor b stellt die Konstanten dieser zusätzlichen Beschränkungen dar und V repräsentiert die dazugehörige Koeffizientenmatrix. Gesamtbetriebliche Beschränkungen sind z.B. die Vorgabe der für jede Zeitperiode $t$ erwünschten Holzvorräte (oder Vermögenswerte), die maximal erlaubten Nutzungen, die erlaubte maximale zeitliche Fluktuation der Nutzungen (im engl. Sprachgebrauch bekannt als even flow), die minimale Fläche an Altbeständen am Ende der Planungsperiode, oder die Vorgabe der räumlichen Konzentration (Harvestereinsatz) bzw. Dispersion (Naturschutz) der Nutzungen. In der Abbildung 1 ist dieses Modell grafisch dargestellt.

Mathematische

Formulierung Grafische Darstellung

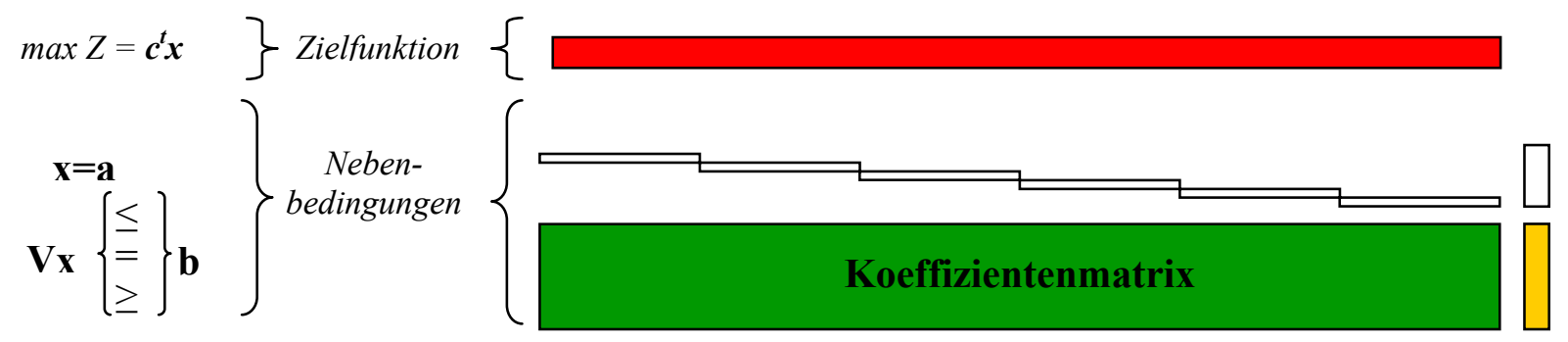

Abbildung 1. Mathematische Formulierung und grafische Darstellung der Zielfunktion und der Nebenbedingungen im Modell der Waldoptionen.

Die Lösung dieses eleganten und sehr einfachen Ansatzes, der früher in den USA sehr populär war, zeigt die Flächenanteile der Optionen, die den Straten zugewiesen werden. Diese Lösung hat den Nachteil, dass sie in einem zweiten Schritt noch in konkrete Handlungsempfehlungen für die Betriebsleitung transformiert werden muss: in welchem Bestand soll wann, wie eingegriffen werden, damit die Ziele und Beschränkungen auf Betriebsebene auch wirklich erfüllt werden? Dieser Arbeitsschritt der Forsteinrichtung ist erheblich arbeitsaufwendiger und methodisch anspruchsvoller als die strategische Typenplanung. Daher ist es sinnvoll, die Planung von Beginn an auf Waldbestände auszurichten. 
Das Modell der Waldoptionen unterstellt, - ähnlich wie das Modell der Stratenoptionen (obwohl im Grundsatz sehr unterschiedlich), - dass eine Waldlandschaft sich mosaikartig aus einzelnen Beständen zusammensetzt. Jeder lagemäßig definierte Bestand $i$ (i=1..I) mit der Fläche $a_{i}$ weist bestimmte Dichte-, Struktur- und Wertmerkmale auf. Für jeden Bestand existieren daher unterschiedliche Möglichkeiten der Entwicklung. Eine Bestandesoption $j\left(j=1 . . J_{i}\right)$ ist eine spezielle Folge natürlicher Bestandesentwicklungen und forstlicher Eingriffe. Die Anzahl der möglichen Optionen kann sehr groß sein. Da in ungleichaltrigen und artenreichen Wäldern nicht nur die Stärke, sondern auch die Art der Eingriffe sehr unterschiedlich sein kann, steigt die Anzahl der möglichen Optionen mit zunehmender Strukturvielfalt der Bestände. Ein Entwicklungspfad $j$ im Bestand $i$ hat, wie im Modell der Stratenoptionen, einen den übergeordneten Zielsetzungen entsprechenden Nutzen pro Flächeneinheit.

Die Theorie der Waldoptionen ist ein denkbar einfacher Ansatz, für den es unterschiedliche technische Lösungen gibt. Sie bietet nicht nur ein effektives Instrumentarium der forstbetrieblichen Steuerung, sondern auch eine fundierte Basis für die fachliche Integration der unterschiedlichen forstlichen Disziplinen, - ein Sammelbecken für die praktische Anwendung vielfältiger Erfahrungen und wissenschaftlicher Erkenntnisse (Kurth et al., 1994). Damit wird gleichzeitig die Aufgabe der Einzel- und der Gesamtplanung erfüllt und die taktische und operationale Ebene der Betriebsführung verknüpft.

Die Steuerung der Waldentwicklung dient der multifunktionalen Waldnutzung. Die Problematik und die Lösungsansätze, vor allem die Methoden der Generierung von Bestandesentwicklungsszenarien unter Verwendung neuer Optimierungsverfahren werden in der vorliegenden Arbeit am Beispiel von Fichten- und Kiefernbeständen dargestellt. 


\section{Untersuchung zur optimalen Steuerung der Waldentwicklung am Beispiel von gleichaltrigen Fichtenbeständen}

\subsection{Einleitung}

\subsubsection{Die Fichte in Deutschland: bisherige Untersuchungen}

Die Fichte (Picea abies Karst.) ist eine der vorherrschenden Baumarten in Deutschland. Nach den Angaben der I. Bundeswaldinventur ist 37\% der Waldfläche Westdeutschlands mit Fichte bestockt. Zudem hat die Fichte mit 43 \% den höchsten Anteil an den Reinbeständen (Bundeswaldinventur 1986-1990). Die langfristige ökologische Waldbauplanung sieht den Fichtenanbau in Reinbeständen nur noch in einem sehr begrenzten Umfang vor. Die Fichte soll künftig vermehrt in Mischbeständen vorkommen. Ihr Flächenanteil wird sich damit voraussichtlich langfristig verringern (Beck et. al., 1997). Sowohl aus klimatischer als auch bodenkundlicher Sicht belegt die Halbschattenbaumart Fichte eine große standörtliche Amplitude. Ihr Lichtbedürfnis hängt von der Nährstoff- und Wasserversorgung ab. Sie ist weitgehend resistent gegen Winterfröste, leidet jedoch in gefährdeten Lagen unter Spätfrost. Ihre Ansprüche an die Sommerwärme sind gering. Ihre Wuchsleistung wird vom Wasserhaushalt erheblich stärker bestimmt als vom Nährstoffgehalt des Bodens. Sie gedeiht in Deutschland bei Jahresniederschlägen zwischen 600 und $3000 \mathrm{~mm}$. Besonders empfindlich ist sie gegenüber Sommerdürre. Unter günstigen Klimabedingungen erreicht die Fichte ihre beste Wuchsleistung auf tiefgründigen, bodenfrischen, sandig-lehmigen, braunerdeartigen Böden mittlerer Basenversorgung (Kramer, 1988).

Über das Wachstum der Fichte im Reinbestand liegt bereits eine Vielzahl von Veröffentlichungen vor. Die ersten Ertragstafeln für Fichte erschienen am Ende des 19. bzw. Anfang des 20. Jahrhunderts (Schwappach, 1890; Schiffel, 1904; Grundner, 1913). Sie enthalten die grundsätzlichen Erkenntnisse aus der waldwachstumskundlichen Forschung, verbunden mit waldbaulichen Zielsetzungen und Vorstellungen über geeignete Behandlungskonzepte ihrer Entstehungszeit (Schübeler, 1997). Ertragstafeln geben lediglich mittlere Erwartungswerte des Wachstums für Großgebiete wieder, ohne die durch Umwelteinflüsse oder durch eine von den Ertragstafelvorgaben abweichende Behandlung bedingten Differenzierungen im Wachstumsmuster zu berücksichtigen. Die ersten Arbeiten, in denen die Beziehungen zwischen Fichtenwuchsleistung und Bodenvegetationstyp untersucht wurden, stammen aus den zwanziger und dreißiger Jahren des 20. Jahrhunderts (z.B. Kötz, 1929; Rubner, 1929; Blanckmeister u. Merz, 1929; Merz, 1931; Schmidt u. von 
Gaisberg, 1936). In der zweiten Hälfte der fünfziger Jahre begann, vor allem in Süddeutschland und in der ehemaligen DDR, die Entwicklung von standortabhängigen Wuchsmodellen, altersabhängigen Korrekturfaktoren für Großgebietsertragstafeln oder gesonderten Standortertragstafeln. Am Anfang der achtziger Jahre traten neuartige Waldschäden mit Vergilbung der Assimilationsorgane, Nekrosen, Verlichtung der Kronen und schließlich dem Tod der betroffenen Bäume auf. Mehrere Arbeiten berichten über Zuwachsverluste durch Verlust oder Schädigung der Assimilationsorgane. In den neunziger Jahren ist in den deutschsprachigen Ländern Europas eine Reihe von statistischen Einzelbaumwuchsmodellen entstanden, die in der forstlichen Praxis Anwendung finden (Pretzsch, 1992; Hasenauer, 1994; Sterba, 1995; Nagel, 1999). Darüber hinaus sind im Laufe der neunziger Jahre die zwei wichtigsten Bestandessimulatoren in Deutschland entstanden: SILVA (Pretzsch u. Kahn, 1998) und BWinPro (Nagel et al., 2002). Diese Waldwachstumssimulatoren sind Planungsinstrumente, die das Wachstum in Reaktion auf unterschiedliche Durchforstungskonzepte für die wichtigsten Baumarten Deutschlands sowohl in Rein- als auch in Mischbeständen prognostizieren und die daher für die Entwicklung waldbaulicher Behandlungsoptionen eingesetzt werden können.

\subsubsection{Wuchsmodelle und ihre Anwendung}

Die waldwachstumskundliche Forschung versucht, Anregungen für effektive Datengewinnung zu geben, praxistaugliche Prognoseinstrumente zu entwickeln und grundlegende Gesetzmäßigkeiten der Walddynamik zu erkennen (Gadow, 2002).

Die Konstruktion von Wachstumsmodellen bedeutet Organisation waldwachstumskundlichen Wissens und Synthese des Kenntnisstandes über Einzelaspekte des Waldwachstums zu einer Vorstellung vom Gesamtsystem. Waldwachstumsmodelle geben der Forstwissenschaft Einblicke in die komplexen Interaktionen zwischen Strukturen und Prozessen in Waldökosystemen und ermöglichen ein tieferes Verständnis des Waldwachstums (Pretzsch, 2001). Außerdem sind Wachstumsmodelle effiziente Hilfsmittel zur Unterstützung der Planung und Entscheidungsfindung auf Bestandes-, Betriebs- und Großregionsebene. Die Modelle des Bestandeswachstums lassen sich nach ihrer Auflösung in drei unterschiedliche Typen einteilen (Clutter, 1963; Munro, 1974; Davis und Johnson, 1987):

1. Bestandesmodelle beschreiben die Entwicklung von Reinbeständen als Reaktion auf unterschiedliche Standortbedingungen und Behandlungsvarianten. Ein 
Bestandesmodell liefert Angaben über Mittelwerte bzw. flächenbezogene Größen z.B. Grundfläche, Stammzahl pro ha (Clutter, 1963; Franz, 1968; Hradetzky, 1972; Rodríguez Soalleiro, 1995).

2. Eine feinere Auflösung liefern Wachstumsmodelle, bei denen Bäume mit ähnlichen Merkmalen zu einem Repräsentativbaum vereint werden. Diese sog. Repräsentativbaummodelle finden Verwendung, wenn Merkmalsverteilungen vorliegen (Gadow, 1987; Römisch, 1983; Gerold, 1990; Lemm, 1991).

3. Die höchste Auflösung und die vielfältigsten methodischen Ansätze finden sich bei den Einzelbaumwuchsmodellen, bei denen die Merkmale für jeden einzelnen Baum bekannt sind, eventuell sogar die Lagekoordinaten (Gadow, 2002). Sie beschreiben das Wachstum einzelner Bäume in Abhängigkeit von deren unmittelbarer Nachbarschaft (Pretzsch, 1992; Sloboda et al., 1993; Hasenauer, 1994; Nagel, 1994; Sterba, 1995).

Mit der Unterstützung der Informatik und ihrer Werkzeuge lassen sich die Wuchsmodelle in EDV-Programme umsetzen; so entstehen die Waldwachstumssimulatoren. Ein Wachstumssimulator bietet die Möglichkeit, unterschiedliche waldbauliche Maßnahmen für einen realen Bestand zu simulieren. Eine Eingriffsfolge wird durchgespielt, bevor sie im Wald umgesetzt wird. Wachstumssimulatoren werden heute nicht nur zur kurzfristigen Prognose bei der Fortschreibung von gespeicherten Inventurdaten eingesetzt, sondern dienen vor allem zur Simulation unterschiedlicher Waldentwicklungspfade in realen oder hypothetischen Beständen (Rodríguez Soalleiro, 1995; Gadow u. Hui, 1999). Insgesamt erweisen sich Szenariosimulationen schon jetzt als ein brauchbares, ergänzendes Instrument in der strategischen Waldbauplanung (vgl. z.B. Spellmann, 1998; Hanewinkel, 2001; Chen et al., 2002; Döbbeler u. Spellmann, 2002; Duschl u. Suda, 2002; Pretzsch, 2002). Wegen der vielfältigen, häufig widersprüchlichen Zielsetzungen der Forstbetriebe ist die Steuerung und Analyse der Waldentwicklung eine anspruchsvolle Aufgabe geworden. Die Waldwachstumsforschung und die forstliche Unternehmensforschung sind Disziplinen, die die Forsteinrichtung unterstützen. Da zahlreiche Planungs- und Entscheidungsaufgaben durch die Anwendung der forstlichen Unternehmensforschung erleichtert und vereinfacht werden, sind die Vorzüge dieser Methoden umso offensichtlicher je mehr die Flächengrößen der Betriebe zunehmen. 


\subsection{Modellierung des Grundflächenwachstums bei der Fichte}

Das Ziel der vorliegenden Arbeit ist die Generierung, Bewertung und Optimierung von Managementoptionen. Um dieses Ziel zu erreichen, sind in einem ersten Schritt Untersuchungen zum Bestandeswachstum erforderlich. Die Veröffentlichungen I und II untersuchen die Grundflächenentwicklung bei der Fichte als Funktion von Bestandesvariablen wie z.B. der Oberhöhe und der Stammzahl. Die untersuchten Bestandesgrundflächenmodelle lassen sich in zwei unterschiedliche Gruppen einteilen:

1. Pfad-invariante algebraische Differenzenform-Modelle (PID-Modelle). Das Merkmal dieser Modelle ist, dass die Grundfläche im Alter $\left(\mathrm{A}_{2}\right)$ als Funktion der Grundfläche, Höhe und Stammzahl in Alter $\left(A_{1}\right)$ und Höhe und Stammzahl im Alter $\left(A_{2}\right)$ geschätzt wird.

2. Differentialgleichungen zur Schätzung des jährlichen Grundflächenzuwachses (DIFModelle). Beispiele dafür sind die auf dem state space approach basierenden Modelle von García (1994), Rodríguez Soalleiro (1995) und Kvist Johansen (1999).

Die Veröffentlichung I beschäftigt sich mit den PID-Modellen. Das Ziel der Arbeit ist es, die Eignung verschiedener PID-Modelle für durchforstete, gleichaltrige Fichtenbestände zu vergleichen. Die Daten zur Parametrisierung der Modelle stammen aus 19 Fichtenversuchsflächen ${ }^{1}$. Insgesamt stehen 228 Beobachtungsintervalle für die Variablen Alter, Oberhöhe, Stammzahl, und Grundfläche zwischen zwei forstlichen Eingriffen zur Verfügung.

Die folgenden Modelle wurden in dieser Arbeit untersucht: Schumacher (1939), Pienaar und Shiver (1986), Souter (1986), Gadow und Hui (1993, 1999) und Forss (1994). Wie die Tabelle I.4 zeigt, gibt es zwischen den einzelnen Modellen lediglich geringe Unterschiede in der Genauigkeit der Anpassung. Die Modelle von Schumacher und Souter haben die besten Ergebnisse bezüglich der Kriterien MRES (mittleres Residuum) und RMSE (Wurzel des mittleren quadratischen Fehlers) gezeigt. Die graphische Darstellung der geschätzten über den beobachteten Werten in Abbildung I.4 zeigt, dass die Unterschiede zwischen den beiden Modellen unerheblich sind. Daher können generell beide Modelle (Gl.1 und Gl.2) zur Schätzung des Grundflächenwachstums empfohlen werden.

\footnotetext{
${ }^{1}$ Die Daten wurden uns freundlicherweise von der Niedersächsischen Forstlichen Versuchsanstalt zur Verfügung gestellt.
} 


$$
\begin{array}{ll}
\ln G_{2}=\alpha+\left(\ln G_{1}-\alpha\right) \cdot \frac{A_{1}}{A_{2}} & \text { Schumacher (1939) } \\
\ln G_{2}=\left(\frac{A_{1}}{A_{2}}\right) \cdot \ln G_{1}+\alpha \cdot\left(1-\frac{A_{1}}{A_{2}}\right)+\beta \cdot S \cdot\left(1-\frac{A_{1}}{A_{2}}\right)+\gamma \cdot\left(\ln N_{2}-\left(\frac{A_{1}}{A_{2}}\right) \cdot \ln N_{1}\right) & \text { Souter (1986) }
\end{array}
$$

wobei $\mathrm{A}_{1}$ und $\mathrm{A}_{2} \quad=$ Alter am Anfang und am Ende der Zeitperiode zwischen forstlichen Eingriffen;

$\mathrm{G}_{1}$ und $\mathrm{G}_{2}=$ Grundfläche im Alter $A_{1}$ und $A_{2},\left(\mathrm{~m}^{2} / \mathrm{ha}\right)$;

$\mathrm{S}=$ Standortbonität;

$\mathrm{N}_{1}$ und $\mathrm{N}_{2} \quad=$ Stammzahl im Alter $\mathrm{A}_{1}$ und $\mathrm{A}_{2}$;

$\alpha, \beta$ und $\gamma \quad=$ Modellparameter.

Das Ziel der Veröffentlichung II ist es, die Eignung verschiedener DIF-Modelle für durchforstete, gleichaltrige Fichtenreinbestände zu prüfen und eine vergleichende Analyse zwischen PID- und DIF-Modellen durchzuführen. Zu den früheren deutschen Beiträgen im Zusammenhang mit der Anwendung von DIF-Modellen zählen die Arbeiten von Sloboda (1971) und Hradetzky (1972). Die für diese Untersuchung verwendeten Daten stammen aus 87 Fichtenversuchsflächen. Insgesamt stehen 649 Beobachtungswerte mit Alter, Höhe, Stammzahl, Grundfläche am Anfang und am Ende der Zeitperiode zwischen zwei forstlichen Eingriffen zur Verfügung. In Tabelle II.1 sind die durchschnittlichen Werte einiger Bestandesparameter sowie eine Sortierung der Daten nach Dauer des Messintervalls zwischen den Messungen dargestellt.

Die folgenden DIF-Modelle wurden untersucht: García (1994), Rodríguez Soalleiro (1995) und Kvist Johannsen (1999). Nach dem Wert des Korrelationskoeffizienten, sowie der Wurzel des mittleren quadratischen Fehlers (RMSE) zeigt sich das Modell von Rodríguez Soalleiro (G1. 3) als das beste.

$$
\frac{d G}{d t}=a \cdot G^{b} \cdot t^{c}
$$

wobei $\mathrm{dG} / \mathrm{dt}=$ laufender Grundflächenzuwachs ( $\mathrm{m}^{2} /$ ha $\left.\cdot J a h r\right)$;

$$
\begin{array}{ll}
\mathrm{G} & =\text { Grundfläche }\left(\mathrm{m}^{2} / \mathrm{ha}\right) ; \\
\mathrm{t} & =\text { Alter (Jahre); }
\end{array}
$$

$\mathrm{a}, \mathrm{b}$ und $\mathrm{c} \quad=$ Modellparameter. 
Ein weiteres Ziel der Veröffentlichung II ist die vergleichende Analyse zwischen den Modellen des PID- und DIF-Typs. Um diese durchzuführen, ist es nötig, die DIF-Modelle in die Pfad-invariante algebraische Differenzenform zu transformieren. Die Ergebnisse dieser Untersuchung haben gezeigt, dass die PID- Modelle eine bessere Anpassung als die DIFModelle ergeben. Die Begründung dafür ist, dass der Korrelationskoeffizient zwischen zwei Variablen (z.B. $G_{2}$ und $G_{1}$ ) immer höhere Werte als der Korrelationskoeffizient zwischen einer der beiden Variablen und der Differenz der Variablen annimmt.

\subsection{Modellanwendung: Bewertung und Optimierung von Behandlungsoptionen}

Die Veröffentlichung III stellt eine Lösung für das Integrationsproblem der Einzel- und Gesamtvornutzungsplanung am Beispiel eines Fichtenwaldes vor. Die Daten für diese Untersuchung stammen aus 38 Fichtenbeständen des Waldteils Winnefeld Süd im Solling. In Tabelle III.1 sind die Anfangsdaten für jeden Bestand zusammengefasst.

Die Aufgabe der Einzelplanung ist die Bestimmung der Bestandeseingriffe, während die Gesamtplanung sich mit der Bestimmung der besten Managementoptionen für jeden Bestand unter Berücksichtigung gesamtbetrieblicher Beschränkungen und Zielsetzungen beschäftigt (Wikström, 2000). Zur Generierung der Managementoptionen wurde ein von Vilčko und Gadow (2002) entwickeltes Fichtenwuchsmodell verwendet, das auf Basis der Arbeiten von Schübeler (1997), Gurjanov et al. (2000) und Sánchez Orois et al. (2001) entstanden ist. Tabelle III. 2 zeigt die grundlegenden Funktionen dieses Wuchsmodells. Um forstliche Begriffe wie Stärke und Art der Durchforstung in eine quantitative Entnahmevorschrift zu übertragen, wurden die von Spellmann et al. (1999) vorgeschlagenen Durchforstungskriterien (s. Tabelle III.3) verwendet. Mit Hilfe der von Lappi (2002) entwickelten Software Jakta wurden die Optionen generiert. Jakta ist ein allgemeines Statistikprogramm, das u.a. lineare, nicht lineare und nicht-parametrische Regressionen umfasst. Des Weiteren enthält es ein Unterprogramm zur Simulation forstlicher Managementplanung. Die Simulation wurde für vier 5-jährige Planungsperioden durchgeführt, so dass der Planungshorizont 20 Jahre beträgt. Zur Vereinfachung des Simulationsmodells wurden zwei mögliche Behandlungsoptionen simuliert: Niederdurchforstung oder keine Durchforstung. Obwohl diese Behandlungsoptionen nicht der gebräuchlichen Behandlung von Fichtenbeständen entsprechen, dienen sie dazu, diese Methode der Optionengenerierung zu demonstrieren. In Zukunft wäre es interessant, andere Durchforstungskonzepte im 
Simulationsmodell zu integrieren. Da die Simulation aus vier 5-jährigen Planungsperioden besteht sind insgesamt $2^{4}=16$ Managementoptionen pro Bestand generierbar. Abbildung III.1 stellt die Anzahl der von Jakta generierten möglichen Optionen für einen Bestand dar.

Die Bewertung der generierten Optionen erfolgt nach dem ökonomischen Kriterium des Kapitalwertes (Gl. 4). Diese Gleichung ist eine Weiterentwicklung der sog. Zeitfenstermethode der dynamischen Investitionsrechung (Hille et al., 1999). Nach dieser Formel ergibt sich der diskontierte Jetztwert einer Eingriffsfolge aus der Summe der diskontierten Zahlungsüberschüsse, dem diskontierten Abtriebswert und dem diskontierten Bodenertragswert.

$$
K W_{t-t_{0}}=\frac{\sum_{i=t_{0}}^{t} Z \ddot{U}_{i}(i+1)^{\left(t-t_{i}\right)}}{(i+1)^{\left(t-t_{0}\right)}}+\frac{A_{t}}{(i+1)^{\left(t-t_{0}\right)}}+\frac{B_{t}}{(i+1)^{\left(t-t_{0}\right)}}
$$

wobei:

$$
\begin{array}{ll}
K W=\text { Kapitalwert; } \\
t & =\text { Alter, in dem ein Durchmesser von mindestens } 40 \mathrm{~cm} \text { erreicht wird; } \\
t_{0}= & \text { Bestandesalter am Anfang der Planungsperiode; } \\
Z \ddot{U}_{i}= & \text { Zahlungsüberschüsse im Jahr } i, \mathrm{~d} . \text { h. alle Einzahlungen minus alle } \\
& \text { Auszahlungen zum Zeitpunkt } i ; \\
A_{t}= & \text { erntekostenfreier Abtriebswert des Bestandes zum Zeitpunkt } t \\
i & =\text { Zinsrate }(\mathrm{r}=4 \%) \\
B_{t} & =
\end{array}
$$

Für diese Berechnung wurde ein Holzerlös von $80^{2} € / \mathrm{m}^{3}$ und ein Zinssatz von 4\% unterstellt. Um eine genaue Bewertung der Optionen durchzuführen, sollten die Holzerntekosten und Fixkosten berücksichtigt werden. In dieser Untersuchung fehlen leider genaue Kostenangaben. Da das Ziel der Arbeit darin besteht, die Methode der Optimierung und Optionengenerierung zu untersuchen, wurden die Kosten vernachlässigt. Der hier ermittelte Wert, der für die Optimierung verwendet wurde, entspricht dem Wert des stehenden Bestandes. Außerdem wurde eine Untersuchung der Auswirkung des Holzpreises und verschiedener Zinssätze durchgeführt; wobei eine Schwankung des Holzpreises um $\pm 20 \%$ und zwei verschiedene Zinssätze ( $\mathrm{i}=1 \%$ und $\mathrm{i}=7 \%$ ) durchgespielt wurden.

Das Optimierungsproblem der zeitlich-räumlichen Planung wurde mit Hilfe des Softwarepakets JLP (Lappi, 1992) gelöst. Diese Software findet die optimale Kombination von Managementoptionen mit Hilfe der Methode der linearen Programmierung. Das Ziel der Optimierung bestand darin, die Summe der Kapitalwerte $\mathrm{zu}$ maximieren, mit der 
Beschränkung, dass die Zahlungsüberschüsse im Laufe der Planung nicht abnehmen dürfen (s. Gl. III.16-19). Die Abbildungen III.4 - 7 stellen graphisch die optimalen Raum-ZeitMuster dar; es wird deutlich gezeigt, wann und wo ein Eingriff durchgeführt werden sollte. Die in dieser Untersuchung ermittelte Summe des Kapitalwertes für alle Bestände des Waldes Winnefeld Süd beträgt $780981 €$ (Holzpreis von $80 € / \mathrm{m}^{3}$ und Zinssatz $=4 \%$ ) in der optimalen Behandlungsvariante.

\footnotetext{
${ }^{2}$ Der verwendete Holzerlös stammt aus einer Untersuchung von Knoke et al. (2001) für Fichtenbestände in Bayern; im Solling wären die Holzerlöse niedriger (Möhring, pers. Mitteilung, 2003).
} 


\section{Untersuchung zur optimalen Steuerung der Waldentwicklung am Beispiel von ungleichaltrigen Kiefernbeständen in Galicien}

\subsection{Einleitung}

\subsubsection{Pinus pinaster Ait: Ihre Bedeutung für die Forstwirtschaft Galiciens}

Die Comunidad Autónoma Galicia (autonome Region Galicien) liegt in Nordwesten Spaniens und umfasst eine Fläche von rund 29.400 qkm. Dies entspricht etwa 5,8\% der gesamten Fläche der Iberischen Halbinsel. Die Einwohnerzahl liegt bei knapp 3 Millionen. Im Norden grenzt Galicien an das kantabrische Meer, im Westen an den Atlantischen Ozean; im Süden grenzt es an Portugal und im Osten an die spanischen Regionen Castilla-León und Asturias. Galicien gliedert sich in die vier Provinzen: Pontevedra, A Coruña, Lugo und Ourense.

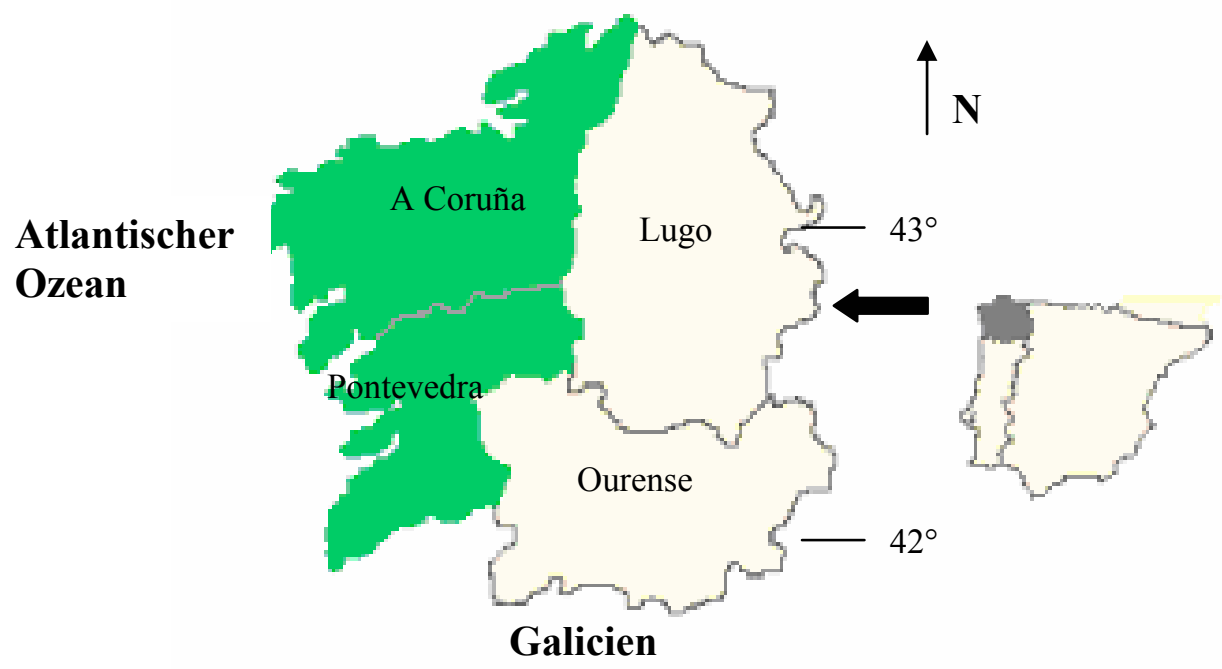

Abbildung 2. Geographische Lage Galiciens in Nordwest- Spanien.

Das Klima der Region wird vom Atlantik geprägt und ist ausgesprochen mild. Die durchschnittliche Temperatur liegt bei $12^{\circ}$ und der durchschnittliche jährliche Niederschlag reicht von 1000 bis 2500 Millimeter; im Gegensatz zum Mittelmeerklima gibt es keinen trockenen Sommer. Als Grundgestein liegen hauptsächlich Granit, Schiefer und Tonschiefer vor. Die daraus entstandenen Böden sind sauer und von minderer Ertragskraft. Aufgrund der geologischen und klimatischen Bedingungen sowie der menschlichen Aufforstungen ist Galicien die waldreichste Region Spaniens. Die Waldfläche beträgt 1.424 .727 ha, somit ist 48\% der Fläche Galiciens mit Wald bestockt (DGCONA, 2000). In den letzten 11 Jahren hat die Waldfläche um 13\% zugenommen. Die Baumarten mit der größten wirtschaftlichen 
Bedeutung sind Kiefer (Pinus pinaster Ait., Pinus radiata, Pinus sylvestris) und die in der Mitte des 19. Jahrhunderts eingeführte Baumart Eucalyptus globulus.

Pinus pinaster Ait. ist die Kieferart, die die wichtigste Rolle in der Forstwirtschaft Galiciens spielt. Von der gesamten Waldfläche Galiciens ist ca. 47\% (620.000 ha) mit Seestrandkiefer in Rein- oder Mischbeständen bestockt. Nach Angaben der III. Waldinventur ist in den letzten 11 Jahren die mit Seestrandkiefer bestockte Fläche um 5\% gestiegen. Der gesamte Vorrat dieser Baumart beträgt 50 Millionen $\mathrm{m}^{3}$ und das jährliche Erntevolumen beträgt ca. 2.5 Millionen $\mathrm{m}^{3}$.

\subsubsection{Bisherige Untersuchungen zum Wachstum der Pinus pinaster in Galicien}

Wegen der großen Bedeutung der Baumart Pinus pinaster für die Forstwirtschaft Galiciens hat man im Laufe der Jahre bereits zahlreiche Arbeiten im Bereich der waldwachstumskundlichen Forschung veröffentlicht. Eine der ersten Arbeiten von Echevarría und De Pedro (1948) beschäftigt sich mit der Entwicklung von klassischen Ertragstafeln. Das von Rodríguez Soalleiro et al. (1994) entwickelte Wachstums- und Ertragsmodell ermöglicht die Simulation und Beurteilung unterschiedlicher Eingriffe in gleichaltrigen Reinbeständen. Dieses Modell wurde im EDV-Programm PINASTER umgesetzt (Rodríguez Soalleiro 1995; Álvarez González et al., 1999). Seit der zweiten Hälfte der neunziger Jahre richtete sich das Interesse der modellorientierten Waldwachstumsforschung in Galicien auf die Beschreibung der Struktur der Bestände und der Analyse ihrer Dynamik (Schröder, 2000). Zur Modellierung der Durchmesserverteilung in homogen aufgebauten, gleichaltrigen Reinbeständen verwendete Álvarez González (1997) die zwei-parametrige Weibull-Funktion. Ein Beitrag zur Weiterentwicklung der Prognoseinstrumente für Pinus pinaster sind die in der Dissertationsarbeit von Schröder (2000) konzipierten Einzelbaummodelle für Reinbestände.

\subsection{Modellierung des Wachstums ungleichaltriger Kiefernbestände}

Als Ergebnis des von den Privatbesitzern angewendeten Managements sind in Galicien Kieferbestände mit unregelmäßiger Struktur entstanden. Die privaten Wälder haben im Durchschnitt eine Fläche unter einem Hektar. Das Management umfasste normalerweise eine Art von Hochdurchforstung des Bestandes, indem nur die besten und die größten Bäume entnommen wurden, wenn eine Bareinnahme von dem Besitzer benötigt wurde. Die Bäume, 
die ein schlechtes Wachstum und niedrige Qualität zeigten, wurden im Bestand belassen (Molina, 1988). Das Resultat ist ein unterbestockter ungleichaltriger Wald, dem es in vielen Fällen an kommerziell wertvollen Bäumen mangelt (Romero, 1992).

Eine spezielle Art von Kiefernwald mit einer Mischung aus Laubbaumarten und Kiefern unterschiedlichen Alters hat sich aus diesem Management, besonders auf guten Standorten, ergeben. Die Fläche dieser Wälder beträgt 87000 ha im Küstengebiet von Galicien. Die traditionelle Ansicht der Forstverwaltung betrachtet diese Wälder als "vernunftwidrig" und schätzt, dass die Produktivität nur ein Drittel der reinen gleichaltrigen Bestände ausmacht (Xunta de Galicia 1992). Jedoch waren Wachstums- und Ertragsdaten für diese Mischwälder nicht vorhanden. Bisher blieb die Möglichkeit unerforscht, ein aktives Management für diese Wälder durchzuführen. Das Ziel der Veröffentlichung IV ist die Beschreibung dieser Wälder, die Entwicklung eines Wachstumsmodells für ungleichaltrige Bestände und schließlich die Entwicklung von gut bestockten sowie unterbestockten Beständen unter Anwendung verschiedener waldbaulichen Behandlungen zu simulieren.

Die für diese Untersuchung verwendeten Daten stammen aus 213 Stichproben der spanischen Waldinventur in den Provinzen von A Coruña und Pontevedra. Aus diesen Daten wurde ein Matrixwuchsmodell nach dem von Buongiorno et al. (1995) vorgeschlagenen Verfahren entwickelt (s. Gl. IV.1). Für die Prognose der Verjüngung und der Mortalität wurden logistische Modelle verwendet. Die Auswahl der Variablen wurde mit Hilfe der Prozedur Stepwise-SAS durchgeführt. Das Modell erkennt 10 Durchmesserklassen und zwei Gruppen von Baumarten: Seestrandkiefer und Laubbaumarten (hauptsächlich Quercus robur). Zur Schätzung der Modellparameter kam die Routine PROC REG (SAS) zum Einsatz. In den Tabellen IV.2 und IV.3 werden die Ergebnisse der Anpassung gezeigt. Mit Hilfe des Modells wurden drei Simulationen durchgeführt:

1. Eine Simulation ohne Durchforstungen in einem gut bestockten Bestand.

2. Die Simulation eines nachhaltigen Regimes für einen gut bestockten Bestand.

3. Wiederaufbauregime für einen unterbestockten Bestand.

Das Ergebnis der Simulation ohne Durchforstungen (s. Abb. IV.3) zeigt eine Abnahme der Bäume in der kleinsten BHD-Klasse und eine Zunahme in der $60 \mathrm{~cm}$ BHD-Klasse. Die Grundfläche nach den vier 5-jährigen Simulationsperioden stieg um 72,5\% an. 
Die Simulation eines nachhaltigen Regimes (Abb. IV.4) führt zu einer ausgewogenen Durchmesserverteilung. Das durchschnittliche ausscheidende Volumen innerhalb einer 5jährigen Periode beträgt $37,1 \mathrm{~m}^{3} /$ ha.

Im unterbestockten Bestand wurden gemäßigte Durchforstungen (nur 40\% des jährlichen Zuwachses wird geerntet) simuliert, um den Vorratsaufbau des Bestandes zu beschleunigen. Das Ergebnis zeigt eine Zunahme der Grundfläche von $7 \mathrm{~m}^{2} /$ ha bis zu 15 $\mathrm{m}^{2} /$ ha und ein Volumen am Simulationsende von $114,1 \mathrm{~m}^{3} / \mathrm{ha}$.

\subsection{Modellanwendung: Bewertung und Optimierung von Behandlungsoptionen}

Heutzutage bekommen Schutz- und Erholungsfunktion durch die Gesellschaft in vielen Regionen von Zentraleuropa größere Bedeutung als Holzproduktion (Dunkel et al., 1994). Es liegt auch ein wachsender Schwerpunkt auf biologischen Funktionen, wie Artenanzahl, Lebensraum und Prozesserhaltung (Sturm, 1995). Außerdem sind Wälder nachwachsende Ressourcen und erzeugen wesentliche Rohstoffe mit wenig Energieverbrauch. Die Anwendung des kahlschlagsfreien, selektiven Nutzungssystem, das gemischte ungleichaltrige Bestände, standortsangepasste Baumarten und Selektivnutzungen bevorzugt, ist das dominierende Managementsystem in Zentraleuropa geworden (Reininger, 1987; Griesel u. Gadow, 1995; Seydack et al., 1995; Butter, 1998; Rautiainen, 1999; Pommerening, 2001).

Das in der Veröffentlichung IV entwickelte Modell kam zur Generierung von Managementoptionen für ungleichaltrige Kiefernwälder in Galicien zum Einsatz. Mit Hilfe dieses Wuchsmodells wurden in der Veröffentlichung $\mathbf{V}$ zwei Ziele erfüllt:

- Den optimalen Vorrat und Nutzungszyklus zu bestimmen.

- Eine vergleichende Analyse zwischen dem Kahlschlagsystem (Rotation Forest Management) und dem kahlschlagsfreien, selektiven Nutzungssystem (Continuous Cover Forestry) durchzuführen.

Einer der Vorteile des kahlschlagsfreien, selektiven Nutzungssystems ist die Schutzfunktion gegen Bodenerosion, wegen der ununterbrochenen Bestockung, außerdem wird oft die Landschaftsästhetik verbessert und die negativen Wirkungen der Habitatszerteilung werden minimiert (Guldin, 1996; Saunders et al., 1991). Des Weiteren hat die Anwendung des kahlschlagsfreien, selektiven Nutzungssystems in den galicischen Kiefernwäldern unter bestimmten Bedingungen gegenüber dem Kahlschlagsystem ökonomische Vorteile für den 
Waldbesitzer. Das Dauerwaldsystem bringt kleine aber regelmäßige Einkommen ein, während das Kahlschlagsystem große aber unregelmäßig verteilte Einkommen abwirft.

Verschiedene Managementoptionen, die fünf unterschiedliche Nutzungszyklen (3, 4, 5, 6 und 7-jährigen Nutzungszyklen) und unterschiedliche Vorratsniveaus $\left(73 \mathrm{~m}^{3} / \mathrm{ha}, 103\right.$ $\mathrm{m}^{3} / \mathrm{ha}, 119 \mathrm{~m}^{3} / \mathrm{ha}$ und $133 \mathrm{~m}^{3} / \mathrm{ha}$ ) analysieren, wurden mit dem Wuchsmodell generiert. Tabelle V.1 fasst das für die insgesamt 20 generierten Optionen ausscheidende Volumen zusammen.

Um die Optionen miteinander vergleichen zu können, wurde das ökonomische Kriterium des Bodenertragswertes (LEV) verwendet. Hierfür kam die von Chang (1981) vorgeschlagene Funktion zum Einsatz:

$$
L E V=\frac{V(Q(t, g))-v(g)-k}{e^{r t}-1}-v(g)-k
$$

wobei:

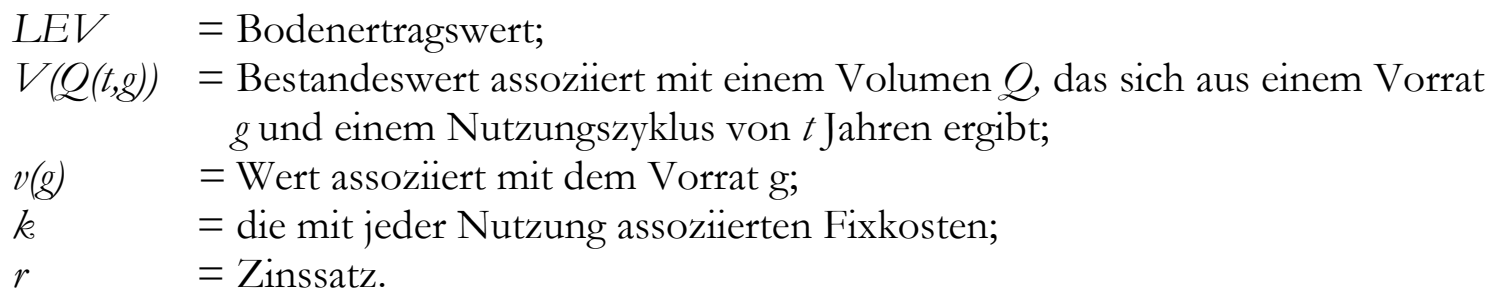

Die Tabelle V.2 zeigt die Einkommen und die Bodenertragswerte für die unterschiedlichen Kombinationen von Vorrat und Nutzungszyklus, wobei ein Zinssatz von 4\% und Fixkosten von 1,5€/ha verwendet wurden. Als beste Option ergibt sich die Kombination eines 5jährigen Nutzungszyklus und eines Vorrats von $133 \mathrm{~m}^{3} / \mathrm{ha}$. In diesem Fall beträgt der Bodenertragswert 6083,82 €/ha. Die Kombination eines 7-järhriges Nutzungszyklus und eines Vorrats von $73 \mathrm{~m}^{3} /$ ha hat sich als die ungünstigste Option erwiesen; in diesem Fall beträgt der Bodenertragswert 2563,13€/ha.

Zur Untersuchung der Auswirkung des Holzpreises wurde eine Senkung des Preises um 20\% durchgespielt. Wie in der Tabelle V.4 zu ersehen ist, bleiben das optimale Vorratsniveau und der Nutzungszyklus unverändert. Ebenfalls wurde der Einfluss einer Veränderung des Zinssatzes auf die optimale Lösung untersucht. Die Tabelle V.3 fasst die Ergebnisse für einen Zinssatz von 1\% und 7\% zusammen. Für einen Zinssatz von 1\% bleiben das optimale Vorratsniveau sowie der Nutzungszyklus unverändert, aber wenn ein 
Zinssatz von $7 \%$ verwendet wird, sinkt das optimale Vorratsniveau auf $73 \mathrm{~m}^{3} /$ ha und der optimale Nutzungszyklus auf 4 Jahre. Erwartungsgemäß, wenn der Zinssatz zunimmt, steigen auch die mit dem Verbleiben des Vorrats assoziierten Opportunitätskosten, aus diesem Grund sinkt das Vorratsniveau. Ebenfalls, je höher der Zinssatz ist, desto kürzer wird der Nutzungszyklus.

Anschließend wurde ein Vergleich zwischen dem kahlschlagsfreien, selektiven Nutzungssystem und dem Kahlschlagsystem in gleichaltrigen Kiefernwäldern durchgeführt. Zwei Bestände mit unterschiedlicher Bonität und zwei unterschiedliche Managementoptionen, die häufig in der Praxis verwendet werden (Rodríguez et al., 2000), wurden untersucht. Im Kahlschlag-, wie im selektiven Nutzungssystem, ist das Ziel, den Bodenertragswert (LEV) zu maximieren:

$$
L E V=\frac{V(t)+\sum_{i=1}^{n} H_{i} e^{r\left(t-t_{i}\right)}-C e^{r t}}{e^{r t}-1} \rightarrow \max
$$

wobei:

LEV = Bodenertragswert;

$H_{i} \quad=$ Einkommen aus der $i$. Durchforstung;

$V(t) \quad=$ Wert eines Bestandes vom Alter $t$,

$C \quad=$ Begründungskosten;

$r \quad=$ Zinssatz.

Für einen Bestand auf einem guten Standort $\left(\mathrm{SI}^{3}=16\right)$, wo Niederdurchforstungen im Alter 12, 17, 22 und 27 und Kahlschlag im Alter 35 durchgeführt wurden, beträgt der Bodenertragswert 9049,63 €/ha. Bei einem weniger intensiven Management mit Durchforstungen im Alter 13, 18, 23 und Kahlschlag im Alter 35 beträgt der Bodenertragswert 7948,23€/ha.

Für einen Bestand auf einem schlechteren Standort $(\mathrm{SI}=13)$, wo ein intensives Management angewendet wurde (Durchforstungen im Alter 12, 17, 22, 27 und Kahlschlag im Alter 35) beträgt der Bodenertragswert 6030,22 €/ha. Bei dem weniger intensiven Management mit Durchforstungen im Alter 15, 20, 25 und Kahlschlag im Alter 35 beträgt der Bodenertragswert 5646,38 €/ha.

\footnotetext{
${ }^{3}$ SI- Index für die Standortbonität, entspricht der Oberhöhe des Bestandes bei einem Bezugsalter von 20 Jahren.
} 
Auf den guten Standorten ist der Bodenertragswert des Kahlschlagsystems um 48\% höher als bei dem kahlschlagsfreien, selektiven Nutzungssystem. Aber auf schlechteren Standorten ist der Bodenertragswert des selektiven Nutzungssystems leicht höher als bei dem Kahlschlagsystem.

\section{Diskussion}

\subsection{Anwendung der entwickelten Modelle.}

Waldwachstumskundliche Modelle müssen bei ihrer Anwendung grundsätzlich bezüglich ihrer Erklärungsebene und den Genauigkeitsanforderungen hinterfragt werden (Pretzsch, 2001). Die Gewährleistung einer ausreichenden Prognosegenauigkeit und Sensitivität zur Abbildung alternativen Waldentwicklungspfade eines Modellsystems ist eine Voraussetzung, damit das Modellsystem in der forstlichen Praxis Akzeptanz finden kann (Albert u. Guericke, 2003).

Die in der Veröffentlichung $\mathbf{I}$ untersuchten Modelle wurden mit Hilfe eines unabhängigen Datensatzes validiert. Alle Modelle zeigen geringe RMSE (Wurzel des mittleren quadratischen Fehlers) und hohe Korrelationskoeffizienten (Ryŷ). Dieses Ergebnis bedeutet nicht, dass alle Modelle eine akkurate Schätzung der Grundfläche ermöglichen. Die Validierung hat gezeigt, dass insbesondere die Gleichungen I.4 und I.5 erhebliche systematische Verzerrungen aufweisen. Die von Schumacher und Souter vorgeschlagenen Modelle (Gl. I.6 und I.8) zeigen diesbezüglich bessere Ergebnisse. Obwohl in Bezug auf die Prognosegenauigkeit beide Modelle zur Schätzung der Grundfläche empfohlen werden könnten, hat das Modell von Schumacher den Vorteil, dass nur ein Parameter und zwei unabhängige Variablen (Grundfläche und Alter) notwendig sind.

Der Korrelationskoeffizient (Ryŷ), die Wurzel des mittleren quadratischen Fehlers (RMSE) sowie das mittlere Residuum (MRES) wurden als Kriterien zur Evaluierung der Anpassungsgüte der in der Veröffentlichung II analysierten DIF-Modelle verwendet. Obwohl für die Parametrisierung der DIF-Modelle jährlichen Änderungsraten besser geeignet wären, wurden, wegen des Mangels an jährlichen Daten, die verfügbaren Wachstumsraten für drei-, vier- und fünfjährige Zeitintervalle verwendet. Eine Validierung mit einem unabhängigen Datensatz war in diesem Fall nicht möglich. Das Modell von Rodríguez (Gl. II.5) zeigt die beste Anpassungsgenauigkeit. Ein Grund dafür ist die Altersabhängigkeit, da sich eine deutliche Beziehung zwischen Alter und Grundfläche nachweisen lässt. 
Um die DIF-Modelle mit den PID-Modellen vergleichen zu können, wurden die Differentialgleichungen in die Pfad-invariante algebraische Differenzenform transformiert. Die Tabellen II.3 und II.5 zeigen geringe Unterschiede zwischen den Parameterwerten der DIF- und der in die PID-Form umgewandelten Modelle. Dieses Ergebnis war zu erwarten, da die durchgeführte Umwandlung der Gleichungen in die PID-Form eine Art Approximation der Integrale der zugehörigen Differentialgleichungen liefert. Der Unterschied liegt darin, dass es in dem Datensatz geringe Unterschiede hinsichtlich der Dauer des Messintervalls gibt. Wenn sich alle Daten auf ein konstantes Messintervall beziehen würden, wären die geschätzten Modellparameter beinahe identisch.

Obwohl die Werte der Modellparameter ähnlich sind, gibt es einen offensichtlichen Unterschied im Korrelationskoeffizienten (Ryŷ) zwischen den DIF- und den PID-Modellen. Für diesen Unterschied im Korrelationskoeffizienten gibt es eine mathematische Begründung (Lappi, 1993), da der Korrelationskoeffizient zwischen zwei Variablen (z.B. $G_{1}$ und $G_{2}$ ) immer höhere Werte als der Korrelationskoeffizient zwischen einer der beiden Variablen und der Differenz der Variablen annimmt. Um den Vergleich der DIF- und der PID-Modelle zu ermöglichen, ist es nötig, die Differentialgleichungen durch Integration oder numerisch zu lösen. In dieser Arbeit wurde lediglich die Approximation solcher Integrale durch die Umwandlung in PID-Modelle ersetzt. Eine andere Möglichkeit wäre der Vergleich auf der Differential-Ebene durchzuführen aber darauf wurde wegen der aufwendigen Transformation der Gleichungen in dieser Arbeit verzichtet. In zukünftigen Arbeiten wäre eine Verbesserung der Modelle interessant. Neue Ansätze wie z.B. die von García (2003) und García und Ruiz (2003) vorgeschlagene Modelle sollten bei Fichtenbeständen untersucht werden.

Das in der Veröffentlichung IV entwickelte Modell für ungleichaltrige Kiefernbestände in Galicien zeigt ein geringes Bestimmtheitsmaß sowohl für die Regression zur Bestimmung der Ingrowth (Anzahl der Bäume, die in die kleinste BHD-Klasse von $15 \mathrm{~cm}$ aufgenommen werden) als auch für die Regression zur Bestimmung der Upgrowth (Anzahl der Bäume, die zur nächsten BHD-Klasse übergehen). Ein Grund dafür kann den unterschiedlichen Selektivnutzungen, die in den Versuchsflächen angewendet wurden, zugeschrieben werden. Andere Autoren haben bei ähnlichen Forschungsarbeiten analoge Ergebnisse erhalten (Buongiorno et al. 1995; Volin u. Buongiorno, 1996). Eine Validierung des Modells mit einem unabhängigen Datensatz ist nicht möglich, da keine Aufnahme von ungleichaltrigen Beständen zur Verfügung steht. Die Beibehaltung eines Teils der Daten für die Validierung 
wurde nicht durchgeführt, da es sich bei einem durch zufällige Aufteilung erhaltenen Datensatz eben nicht um einen wirklich unabhängigen Datensatz handelt und damit keine echte Validierung möglich ist (Vanclay, 1994; Monserud und Sterba, 1996; Hasenauer, 1999). Eine praktische Anwendung des vorgeschlagenen Managementregimes in Galicien kann empfohlen werden. Wenn die Anwendung dieser Regime fortfahren sollte, würde sich eine ausgewogene Durchmesserverteilung halten. Obwohl der Ertrag niedriger ist als in gleichaltrigen Beständen, ist es wichtig zu betonen, dass bei der Selektivnutzung ein höherer Anteil von Holz mit sehr guten Qualitätsmerkmalen zu erwarten ist. Die hohe Konkurrenz und die geringen Lichtverhältnisse begünstigen die natürliche Ästung und minimieren den Anteil des Kernholzes. Obwohl die Selektivnutzung höhere Kosten und mehr Schaden am verbleibenden Bestand mit sich bringt, gibt es auch andere Vorteile wie z.B. Schutz gegen Bodenerosion und natürliche Ereignisse, Verbesserung der Landschaftsästhetik, Minimierung der negativen Wirkungen der Habitatszerteilung, Verbesserung des Habitates für die Fauna (Matthews, 1989; Baker et al., 1991; Schulte u. Buongiorno, 1998). Eine wichtige zukünftige Aufgabe wäre die Anlage von Versuchsflächen in ungleichaltrigen Beständen und die Gewinnung von Daten deren Untersuchung eine Verbesserung des Modells und die Analyse waldbaulicher Optionen für ungleichaltrige Bestände ermöglichen.

\subsection{Generierung und Optimierung von Managementoptionen}

Das Problem der Generierung und Optimierung von Managementoptionen wurde in der Veröffentlichung III mit Hilfe des Simulators Jakta zur EDV-gestützten Generierung und Bewertung von Optionen und mit Hilfe des Softwarepakets JLP zur optimalen Lösung der zeitlich-räumlichen Planung gelöst. Dieses Problem der Suche nach dem optimalen RaumZeit-Muster ist ein kombinatorisches Problem mit dem sich die Forsteinrichtung schon seit Jahrzehnten auseinandergesetzt hat. Am Anfang der siebziger Jahre kam zur Lösung dieses Problems das von Ware und Clutter (1971) entwickelte Model I zum Einsatz. Andere spezielle Lösungsansätze finden sich u.a. bei Hoganson und Rose (1984) und Öhman (2001).

Ein Grund, der dafür spricht den Simulator Jakta zu verwenden ist, dass er ermöglicht, gleichzeitig eine hohe Anzahl von Managementoptionen für mehrere Bestände mit unterschiedlichen Ausgangszuständen zu generieren und zu bewerten. Mit Hilfe der Methode der linearen Programmierung wurde das Optimierungsproblem gelöst. Zum Einsatz kam das von Lappi (1992) entwickelte System JLP, das ermöglicht, in wenigen Sekunden von den insgesamt 5037 generierten Optionen die optimale Lösung unter Berücksichtigung der gesamtbetrieblichen Zielsetzungen und Beschränkungen zu finden. Die Methode der linearen 
Programmierung hat den Vorteil, dass der Rechenaufwand geringer als bei anderen Optimierungsmethoden z.B. heuristische Methoden ist (Chen u. Gadow, 2002). Außerdem ist es möglich, zahlreiche Beschränkungen zu definieren und bei Änderung der Zielfunktion oder der Beschränkungen lassen sich mit wenig Aufwand neue Szenarien generieren. Einer der Vorteile den heuristischen Methoden der linearen Programmierung gegenüber ist, dass sie schwierige Probleme der Mehrzieloptimierung lösen können. Insbesondere sind die heuristischen Methoden zur Lösung räumlicher Optimierungsprobleme besser geeignet als die Methode der linearen Programmierung (Pukkala, 2002). Die Zielsetzung in dieser Untersuchung war rein ökonomisch; die Zielfunktion bestand darin, den gesamtbetrieblichen Kapitalwert zu maximieren, mit der Beschränkung, dass die Zahlungsüberschüsse im Laufe der Planungsperiode nicht abnehmen dürfen. Obwohl der verwendete Durchforstungsalgorithmus die Nutzungen beschränkt, reicht diese Beschränkung allein nicht aus, um die Nachhaltigkeit über die Planungsperiode hinaus zu gewährleisten. Dazu wären Informationen über die erwartete Verjüngung und (im schlagweisen Betrieb) die Endnutzung notwendig. In zukünftigen Arbeiten wäre es auch interessant, nicht nur ein ökonomisches Kriterium zu betrachten sondern auch andere Aspekte in der Zielfunktion zu berücksichtigen (z.B. die Förderung der Diversität, die Minimierung der Schwankungen des periodischen Erntevolumens, die räumliche Optimierung der Eingriffe) und die Änderungen der optimalen Lösung mit Hilfe einer Sensitivitätsanalyse zu untersuchen.

In der Veröffentlichung $\mathbf{V}$ wurden mit Hilfe des für ungleichaltrige Kiefernbestände entwickelten Wuchsmodells 20 Optionen generiert, die sich aus der Kombination von fünf verschieden Nutzungszyklen (3, 4, 5, 6 und 7-jährigen Nutzungszyklen) und vier verschiedenen Vorratsniveaus $\left(73 \mathrm{~m}^{3} / \mathrm{ha}, 103 \mathrm{~m}^{3} / \mathrm{ha}, 119 \mathrm{~m}^{3} / \mathrm{ha}\right.$ und $133 \mathrm{~m}^{3} / \mathrm{ha}$ ) ergeben. Mit dem Ziel, den Bodenertragswert zu maximieren, wurden die Optionen miteinander verglichen. Als optimales Management zeigt sich die Kombination eines 5-jährigen Nutzungszyklus und eines Vorrats von $133 \mathrm{~m}^{3} / \mathrm{h}$. Der Vergleich aus der ökonomischen Ansicht zwischen dem ungleichaltrigen und gleichaltrigem Management bestätigt, dass auf guten Standorten das Kahlschlagsystem höhere Gewinne einbringt, während auf schlechten Standorten das ungleichaltrige Management leicht bessere Ergebnisse zeigt. Hierbei ist es wichtig zu berücksichtigen, dass wenn die Preise auf Stock niedriger als bei dem Kahlschlagmanagement wären, dann wäre das ungleichaltrige Management deutlich ungünstiger. Deshalb ist es beim ungleichaltrigen Management notwendig, Holz mit sehr guten Qualitätsmerkmalen zu erzielen. Frühere Untersuchungen (Chang, 1981, 1990) haben 
schon bestätigt, dass niedrige Preise und hohe Zinssätze das ungleichaltrige Management begünstigen. Aus dieser Arbeit kann geschlossen werden, dass auf schlechten Standorten sich das selektive Nutzungssystem als besser geeignet zeigt. Bevor ein gleichaltriger Bestand in einen ungleichaltrigen überführt wird, muss der Entscheidungsträger die sich durch die Überführung zusätzlich ergebenden Vorteile berücksichtigen. Damit die Überführung lohnenswert ist, müssen diese Gewinne größer sein als die Differenz der Erträge zwischen dem gleichaltrigen und dem ungleichaltrigen Management. Zum Schluss muss betont werden, dass die Überführung ein anspruchsvolles Thema ist, das eine Verbesserung des Wuchsmodells und ein tiefes Verständnis der Wuchsdynamik in ungleichaltrigen Beständen erfordert. 


\section{Zusammenfassung}

Die vorliegende Arbeit befasst sich mit der optimalen Steuerung der Waldentwicklung am Beispiel von Fichten- und Kiefern-Laubholzmischbeständen. Das Ziel der Untersuchung ist die Generierung, Bewertung und anschließend die Optimierung von Managementoptionen für gleichaltrige Fichtenreinbestände und ungleichaltrige Kiefern-Laubholzmischbestände. Um dieses Ziel zu erreichen, sind in einem ersten Schritt Untersuchungen zum Bestandeswachstum erforderlich.

Zunächst wurde die Grundflächenentwicklung in Fichtenreinbeständen untersucht. Dabei wurden zwei unterschiedliche Gruppen von Bestandesgrundflächenmodellen analysiert und miteinander verglichen: die Pfad-invarianten algebraischen Differenzenform-Modelle (PID-Modelle) und die Differentialgleichungen zur Schätzung des jährlichen Grundflächenzuwachses (DIF-Modelle). Unter den PID-Modellen haben die Gleichungen von Schumacher (1939) und Souter (1986) die besten Ergebnisse bezüglich der Kriterien MRES (mittleres Residuum) und RMSE (Wurzel des mittleren quadratischen Fehlers) gezeigt. Obwohl in Bezug auf die Prognosegenauigkeit beide Modelle zur Schätzung der Grundfläche empfohlen werden könnten, hat das Modell von Schumacher den Vorteil, dass nur ein Parameter und zwei unabhängige Variablen (Grundfläche und Alter) notwendig sind.

Unter den analysierten DIF-Modellen hat sich die Gleichung von Rodríguez Soalleiro (1995) bezüglich des Wertes des Korrelationskoeffizienten, sowie der Wurzel des mittleren quadratischen Fehlers (RMSE) als die Beste erwiesen.

Am Beispiel eines Waldes mit 38 Fichtenbeständen wurde das Problem der Optimierung der Vornutzungen gelöst. Zunächst wurden mit Hilfe des Managementsimulators Jakta zahlreiche Managementalternativen für die einzelnen Bestände generiert und nach dem ökonomischen Kriterium des Kapitalwertes (BEW) bewertet. Schließlich wurde das optimale Raum-Zeit-Muster von Durchforstungsmaßnahmen für den ganzen Forstbetrieb mit Hilfe des Softwarepakets zur linearen Programmierung JLP bestimmt. Zuletzt wurde eine Untersuchung der Auswirkung des Holzpreises und verschiedener Zinssätze durchgeführt; wobei eine Schwankung des Holzpreises um $\pm 20 \%$ und zwei verschiedene Zinssätze ( $\mathrm{i}=1 \%$ und $\mathrm{i}=7 \%$ ) durchgespielt wurden.

Zur Prognose des Wachstums ungleichaltriger Kiefern-Laubholzmischbestände wurde ein Matrixwuchsmodell entwickelt. Dieses Modell wurde verwendet, um verschiedene Managementoptionen für ungleichaltrige Bestände zu simulieren. Nach dem ökonomischen 
Kriterium des Bodenertragswertes erwies sich ein Nutzungszyklus von fünf Jahren und die Erhaltung eines Vorrats von $133 \mathrm{~m}^{3} /$ ha als die beste Alternative. In diesem Fall beträgt der Bodenertragswert 6083,82 €/ha. Der Vergleich zwischen dem selektiven Nutzungssystem und dem Kahlschlagsystem hat gezeigt, dass auf den guten Standorten der Bodenertragswert des Kahlschlagsystems um 48\% höher als beim kahlschlagsfreien, selektiven Nutzungssystem ist; aber auf schlechten Standorten ist der Bodenertragswert des selektiven Nutzungssystems leicht höher.

Schlüsselwörter: Picea abies, Pinus pinaster, Wuchsmodelle, Generierung und Bewertung von Optionen, Optimierung. 


\section{Abstract}

This study deals with the optimisation of the forest development in even-aged spruce and uneven-aged pine stands. The aim of this research is the generation, evaluation and optimization of management options for even-aged spruce stands and uneven-aged pine stands. To achieve this goal it is necessary, in the first place, to utilize the research results in the area of forest growth. First, the development of the basal area in pure spruce stands was examined. Two different types of basal area models were analysed and compared: The path invariant algebraic difference form models (PID models) and the differential equations models for the estimation of the annual basal area increment (DIF models). Among the analysed PID models the equations of Schumacher (1939) and Souter (1986) showed the best performance according to the criteria of the MRES (mean residuum) and RMSE (root mean square error). Although according to their good performance both models can be recommended for estimating basal area, the model of Schumacher has the advantage that only one parameter and two independent variables (age and basal area) are required.

Among the analysed DIF models the equation of Rodríguez Soalleiro (1995) showed the best results according to the criteria of the correlation coefficient and the RMSE (root mean square error).

Using a forest of 38 spruce stands as an example the problem of the management optimization was investigated. Several management alternatives for each stand were generated with the management simulator Jakta. The economic criterion forest value was used for option evaluation. Finally, the optimal space-time pattern of thinnings for the whole forest was determined using the software package JLP for linear programming. A sensitivity analysis for the optimal solution was carried out, considering the timber price and interest rate changes. A variation of the wood price of $\pm 20 \%$ and two different interest rates ( $i=1 \%$ und $i=7 \%$ ) were explored.

To analyse the growth of uneven-aged pine stands a matrix stand growth model was developed. This model was applied to simulate different management options for unevenaged stands. According to the economic criterion of the land expectation value a cutting cycle of 5 years and a growing stock of $133 \mathrm{~m}^{3} /$ ha showed the best economic results. In this case the land expectation value amounts to $6083,82 € / \mathrm{ha}$. The comparison between continuous forest management and rotation forest management showed that on the good sites the land expectation value of the rotation system is around $48 \%$ higher than if the continuous forest 
system were applied; but on poor sites the land expectation value of the continuous forest system is slightly higher than what can be expected under rotation management.

Keywords: Picea abies, Pinus pinaster, growth models, generation and evaluation of options, optimization. 


\section{Literatur}

Albert, M. und Guericke, M., 2003: Zur Prognose waldbaulicher Bahandlungsvarianten in Mischbeständen mit einzelbaumbasierten Durchforstungsmodellen. Forstarchiv (eingereicht).

Álvarez González, J.G., 1997: Análisis y caracterización de las distribuciones diamétricas de Pinus pinaster Ait. en Galicia. Diss. Universidad Politécnica de Madrid, ETS de Ingenieros de Montes, $270 \mathrm{~S}$.

Álvarez González, J.G., Rodríguez Soalleiro, R. und Vega Alonso, G., 1999: Elaboración de un modelo de crecimiento dinámico para rodales regulares de Pinus pinaster Ait. en Galicia. Investigación Agraria, Sist. Rec. For. 8(2):319-334.

Baker, J. B., Guldin, J. M. und Guldin, R. W., 1991: Natural regeneration methods for loblolly and shortleaf pines. For. Farmer 50: 59 - 63.

Beck, O. A., Engell, T., Hennecke, W., Köhler, H., Otto, H. J., Panitz, E., Peiffer, K., Rang, H., Schiers, J., Spellmann, H., Stratmann, J. und Wollborn, P., 1997: Entscheidungshilfen zur Behandlung und Entwicklung von Fichtenbeständen. Merkblatt Nr. 34. Niedersächsische Landesforstverwaltung, 20 S.

Blanckmeister, H., und Merz, M., 1929: Untersuchungen über die Brauchbarkeit von Florentypen im Riesengebirge. AFJZ 105: 344-353.

Buongiorno, J., Peyron, L., Houllier, F. und Bruciamacchie, M., 1995: Growth and management of mixed-species, uneven-aged forest in the French Jura: Implications for economic returns and tree diversity. For. Sci. 41: 397-429.

Butter, D., 1998: Waldbauliche Strategie und Stand des Waldumbaus im sächsischen Staatswald. Forst u. Holz 20 (53): 609-612.

Chang, S. J., 1981: Determination of the Optimal Growing Stock and Cutting Cycle for an Uneven-Aged Stand. Forest Science 27(4): 739-744.

Chang, S. J., 1990: An economic comparison of even-aged and uneven-aged management of southern pines in the mid-South. In: Hickman, C. A., (ed.). Proceeding of the Southern Forest Economics Workshop on Evaluating Even- and All-Aged Timber Management Options for Southern Forest Lands, March 29-30 1990, Monroe, LA. GTR SO-79. USDA Forest Service, Southern Experimental Station, New Orleans, LA, S. 45-52.

Chen, B. W. und Gadow, K. v., 2002: Timber harvest planning with spatial objectives, using the method of simulated annealing. Forstw. Cbl. 121(1): $25-34$.

Chen, B.W., Gadow, K. von, Vilčko, F. und Sánchez Orois, S., 2002: Medium-term planning for sustainable forest management - an example from Northern Germany. Investigación Agraria: Sistemas y Recursos Forestales (eingereicht).

Clutter, J.L., 1963: Compatible Growth and yield Models for Loblolly Pine. For. Sci. 9(3):354-371.

Davis, L. S. und Johnson, K. N., 1987: Forest Management. McGraw-Hill Book Company, New York.

DGCONA, 2000: Avance del Tercer inventario forestal nacional en Galicia. Ministerio de Medio Ambiente. Madrid.

Döbbeler, H. und Spellmann, H., 2002: Methodological Approach to Simulate and Evaluate Silvicultural Treatments under Climate Change. Forstw. Cbl. 121, Supplement 1: 52-69.

Dunkel, K., Elsasser, P., Oesten, G. und Roeder, A., 1994: Wertschätzung des Waldes aus der Sicht der Waldbesucher - Ergebnisse einer Zielgebietsbefragung im Pfälzerwald. Mitt. d. Forstl. Vers. Anst. Rheinld-Pfalz 27/94: 1-72.

Duschl, Ch. und Suda, M., 2002: Simulation of Management Strategies in the Forest Estate Model “Germany". Forstw. Cbl. 121, Supplement 1: 89-107. 
Echevarría, I. und De Pedro, S., 1948: El Pinus pinaster en Pontevedra su productividad normal y aplicación a la celulosa industrial. Bol. IFIE n 38 . Madrid.

Forss, E., 1994: Das Wachstum der Baumart Acacia mangium in Südkalimatan, Indonesien. Magister Diss. Faculty of Forestry, University of Göttingen, 87 S.

Franz, F., 1968: Das EDV-Programm STAOET zur Herleitung mehrgliedriger StandortLeistungstafeln. Manuskriptdruck, München.

Gadow, K. v., 1987: Untersuchungen zur Konstruktion von Wuchsmodellen für schnellwüchsige Plantagenbaumarten. Forstliche Forschungsberichte München, Nr. 77, $147 \mathrm{~S}$.

Gadow, K. v., 2001: Forsteinrichtung - Alternative zur Kontrollstichprobe. Forsteinrichtung für das 21. Jahrhundert. Tagungsbericht zum Kolloquium des Landesforstvereins Sachsen-Anhalt am 7. Februar 2001 in Drei Annen-Hohne.

Gadow, K. v., 2002: Wuchsmodelle für die Forsteinrichtung. Vortragsmanuskript für die Tagung der AG Zustanderfassung und Planung am 16. Oktober 2002 in Eisenach.

Gadow, K. v. und Hui, G. Y., 1993: Stammzahlentwicklung und potentielle Bestandesdichte bei Cunninghamia lanceolata. Centralblatt für das gesamte Forstwesen 110(2):41-48.

Gadow, K. v. und Hui, G. Y., 1999: Modelling forest development. Kluwer, 213 S.

García, O., 1994: The state space approach in growth modelling. Can. J. For. Res. 24:18941903.

García, O., 2003: Dimensionality reduction in growth models: an example. Forest Biometry, Modelling and Information Sciences 1: 1-15.

García, O. und Ruiz, F., 2003: A growth model for eucalypt in Galicia, Spain. Forest Ecology and Management 173: 49-62.

Gerold, D., 1990: Modellierung des Wachstums von Waldbeständen auf der Basis der Durchmesserverteilung. Dissertation. Dresden, 174 S.

Griesel, F. und Gadow, K. v., 1995: Naturgemäßer - naturnaher - ökologischer Waldbau Begriffsbestimmungen, Konzepte und Richtlinien, Anwendungsbeispiele. Internal report, Institute of Forsteinrichtung, Univ. of Göttingen, 20 S.

Grundner, F., 1913: Normalertragstafel für Fichtenbestände. J. Springer, Berlin, 24 S.

Guldin, J. M., 1996: The role of the uneven-aged silviculture in the context of ecosystem management. Western Journal of Applied Forestry 11(1): 4-12.

Gurjanov, M., Sánchez Orois, S. und Schröder, J., 2000: Grundflächenmodelle für gleichaltrige Fichtenreinbestände. Eine vergleichende Analyse. Centralblatt für das gesamte Forstwesen 117 Heft 3/4: 187-198.

Hanewinkel, M., 2001: Neuausrichtung der Forsteinrichtung als strategisches Managementinstrument. AFJZ 172. Jg. 202-211.

Hasenauer, H., 1994: Ein Einzelbaumwachstumssimulator für ungleichaltrige Kiefern- und Buchen- Fichtenmischbestände. Forstliche Schriftenreihe Universität für Bodenkultur, Wien, $152 \mathrm{~S}$.

Hasenauer, H., 1999: Methodische Aspekte bei der Evaluierung von Baummodellen. Deutscher Verband forstlicher Versuchsanstalten, Sektion Ertragskunde. Beiträge zur Jahrestagung in Volpriehausen, 19.-21. Mai 1999, S. 45-53.

Hille, M., Hessenmöller, D., Möhring, B. und Gadow, K. v., 1999: Bewertung waldbaulicher Massnahmen mit Hilfe der Zeitfenstermethode am Beispiel eines 110jährigen Buchenbestandes. IUFRO Proceedings, Prague.

Hoganson, H. M. und Rose, D. W., 1984: A simulation approach for optimal timber management scheduling. For. Sci. 30(1): 220-238. 
Hradetzky, J., 1972: Modell eines integrierten Ertragstafel-Systems in modularer Form. Mitteilungen der Baden-Würtembergischen Forstlichen Versuchs- und Forschungsanstalt, Heft 45.

Knoke, T., Moog, M. und Plusczyk, N., 2001: On the effect of volatile stumpage prices on the economic attractiveness of a silvicultural transformation strategy. Forest Policy and Economics 2(3-4):229 - 240.

Kötz, F., 1929: Untersuchungen über Waldtyp und Standortsbonität im sächsischen Erzgebirge. AFJZ 105: 41-51, 81-97, 121-136.

Kramer, H., 1988: Waldwachstumslehre. Verlag Paul Parey, Hamburg und Berlin, 374 S.

Kurth, H., Gerold, D. und Ulbricht, R., 1994: Forsteinrichtung. Deutscher Landschaftsverlag Berlin GMBH, S. 233-322.

Kvist Johannsen, V., 1999: A growth model for oak in Denmark. Ph. D. Dissertation, Danish Forest and Landscape Research Institute, Hørsholm, Denmark, 197 S.

Lappi, J., 1992: JLP - A linear programming package for management planning. The Finnish Forest Research Institute. Research papers 414 Suonenjoki, 134 S.

Lappi, J., 1993: Metsäbiometrian menetelmiä. Silva Caraelica 24, 190 S.

Lappi, J., 2002: Jakta user's guide (unveröffentlichtes Manuskript).

Lemm, R., 1991: Ein dynamisches Forstbetriebs- Simulationsmodell. Professur für Forsteinrichtung und Waldwachstum der ETH Zürich, $235 \mathrm{~S}$.

Matthews, J. D., 1989: Silvicultural Systems. Oxford Universty Press, Oxford. 284 S.

Merz, M., 1931: Die Florentypen des Erzgebirges und Vogtlandes und ihre Beziehungen zum Wachstum der Fichtenbeständen. Diss. Eberswalde, A. Remppis Verlag, Marbach am Neckar, $80 \mathrm{~S}$.

Molina, F., 1988: Los bosques de Galicia. Actualidad Forestal de Galicia. No. 106-107 Enero-Junio, S. 1-5.

Monserud, R. A. und Sterba, H., 1996: A basal area increment model for individual trees growing in even-aged forest stands in Austria. For. Ecol. Manage. 80:57-80.

Munro, D., 1974: Forest growth models -a prognosis. Growth models for tree and stand simulations. Res. Note 30, Dep. For. Yield Res., Royal College, Stockholm.

Nagel, J., 1994: Ein Einzelbaumwachstumsmodell für Roteichenbestände. Forst und Holz Nr. 3, S. 69-75.

Nagel, J., 1999: Konzeptionelle Überlegungen zum schrittweisen Aufbau eines waldwachstumskundlichen Simulationssystems für Nordwestdeutschland. Schriften aus der Forstlichen Fakultät der Universität Göttingen und der Niedersächsischen Forstlichen Versuchsanstalt Bd. 128: 122 S.

Nagel, J., Albert, M. und Schmidt, M., 2002: Das waldbauliche Prognose- und Entscheidungsmodell BWINPro 6.1. Forst und Holz 57 (15/16):486-493.

Öhman, K., 2001: Forest planning with consideration to spatial relationships. Doctoral Thesis. Swedish University of Agricultural Sciences. Umeå.

Pienaar, L. V. und Shiver, B. D., 1986: Basal area prediction and projection equations for pine plantations. Forest Science 32(3): 626-633.

Pommerening, A., 2001: Continuous cover forestry - chance and challenge for forest science. Institute of Chartered Foresters (ICF) news 1/2001, special feature "Alternatives to clearfelling": S. 8 - 10.

Pretzsch, H., 1992: Konzeption und Konstruktion von Wuchsmodellen für Rein- und Mischbestände. Forstliche Forschungsberichte München, No. 115.

Pretzsch, H., 2001: Modellierung des Waldwachstums. Parey Buchverlag, Berlin, 341 S.

Pretzsch, H., 2002: Application and Evaluation of the Growth Simulator SILVA 2.2 for Forest Stands, Forest Estates and Large Regions. Forstw. Cbl. 121, Supplement 1: 2851. 
Pretzsch, H. und Kahn, M., 1998: Forschungsvorhaben „Konzeption und Konstruktion“ von Wuchs- und Prognosemodellen für Mischbestände in Bayern. Abschlußbericht Projekt W 28, Teil 2 - Methodische Grundlagen, 279 S.

Pukkala, T., 2002: Introduction to multi-objective forest planning. S. 1-19. In: Multiobjective forest planning. Kluwer Academic Publishers. The Netherlands, 207 S.

Rautiainen, O., 1999: Growth dynamics and management of Shorea robusta forests in Southern Nepal. PhD Diss. Faculty of Forestry, University of Joensuu.

Reininger, H., 1987: Zielstärken-Nutzung. Österreichischer Agrarverlag, 163 S.

Rodríguez Soalleiro, R., 1995: Crecimiento y producción de masas forestales regulares de Pinus pinaster Ait. en Galicia. Alternativas selvícolas posibles. Ph D. Dissertation. Escuela Técnica Superior de Ingenieros de Montes. Madrid, 297 S.

Rodríguez Soalleiro, R., Álvarez González, J.G. und Vega Alonso, G., 1994: Piñeiro do país. Modelo dinámico de crecemento de masas regulares de Pinus pinaster Aiton en Galicia. Xunta de Galicia, Serie manuais prácticos 8, 40 S.

Rodríguez, R., Álvarez, J. G. und Schröder, J., 2000: Simulation and comparation of silvicultural alternatives for even-aged Pinus pinaster Ait. stands in Galicia (Northwestern Spain). Annales des Sciences Forestières 57:747-754.

Romero, A., 1992: El sector forestal en Galicia. Economía Política Forestal. Xunta de Galicia. Santiago de Compostela, S. 113-132.

Römisch, K., 1983: Ein mathematisches Modell zur Simulation von Wachstum und Durchforstung gleichaltriger Reinbestände. Dissertation, Tharandt.

Rubner, K., 1929: Bodenvegetation und Höhenbonität in Lehr- und Versuchsrevier Grafrath. Fw. Cbl. 48: 813-827, 860-879.

Sánchez Orois, S., Gurjanov, M. und Schröder, J., 2001: Analyse des Grundflächenzuwachses gleichaltriger Fichtenreinbestände. Allg. Forst- und Jagdzeitung 172(3):51-60.

Saunders, D. A., Hobbs, R. J. und Margules, C. R., 1991: Biological consequences of ecosystem fragmentation: a review. Conserv. Biol. 5(1): 18-32.

Schiffel, A., 1904: Wuchsgesetze normaler Fichtenbestände. Mitt. FBVA 29, 106 S.

Schmidt, H. und Gaisberg, von E., 1936: Untersuchungen über Standort und Ertragsleistung der Fichte in württembergischen Waldgebieten. Mitteilungen der Württembergischen Forstlichen Versuchsanstalt 1936/1, 129 S.

Schröder, J., 2000: Analyse der Wuchsdynamik von Pinus pinaster Ait. in Nordwest-Spanien. Diss. Georg-August-Universität Göttingen, Curvillier Verlag, Göttingen, 71 S.

Schübeler, D., 1997: Untersuchungen zur standorabhängigen Wachstumsmodellierung bei der Fichte. Diss. Georg- August-Universität Göttingen, Curvillier Verlag, Göttingen, $155 \mathrm{~S}$.

Schulte, B. J. und Buongiorno, J., 1998: Effects of uneven-aged silviculture on the stand structure, species composition, and economic returns of loblolly pine stands. For. Ecol. Manage. 111:83-101.

Schumacher, F. X., 1939: A new growth curve and its applications to timber-yield studies. Journal of Forestry 37:819-820.

Schwappach, A., 1890: Wachstum und Ertrag normaler Fichtenbestände. J. Springer, Berlin, $100 \mathrm{~S}$.

Seydack, A.H.W., Vermeulen, W.J., Heyns, H.E., Durrheim, G.P., Vermeulen, C., Willems, D., Ferguson, M.A., Huisamen, J., und Roth. J., 1995: An unconventional approach to timber yield regulation for multi-aged, multi-species forests. II. Application to a South African forest. For. Ecol. Manage. 77: 155-168.

Sloboda, B., 1971: Zur Darstellung von Wachstumsprozessen mit Hilfe von Differentialgleichungen erster Ordnung. Inaugural-Dissertation zur Erlangung der 
Doktorwürde der Forstwissenschaftlichen Fakultät der Albert-Ludwigs-Universität zu Freiburg im Breisgau, 109 S.

Sloboda, B., Gaffrey, D. und Matsumura, N., 1993: Regionale und locale Systeme von Höhenkurven für gleichaltrige Reinbestände. AFJZ 12: 225-228.

Souter, R. A., 1986: Dynamic stand structure in thinned stands of naturally regenerated loblolly pine in the Gerogia Piedmont. PhD Thesis. Universtiy of Georgia. Athens, GA.

Spellmann, H., 1998: Überführung als betriebliche Aufgabe. M. Hanewinkel (Hrsg.), Überführung von Altersklassenwäldern in Dauerwälder. Freiburger Forstliche Forschung, Heft 8: S. 194 - 217.

Spellmann, H., Nagel, J. und Böckmann, T., 1999: Summarische Nutzungsplanung auf der Basis von Betriebsinventurdaten. Allg. Forst- u. J. Ztg. 170(7):122 - 128.

Sterba, H., 1995: PROGNAUS - ein abstandsunabhängiger Wachstumssimulator für ungleichaltrige Mischbestände, DVFF, Sektion Ertragskunde, Jahrestagung, S. 173183.

Sturm, K., 1995: Möglichkeiten, Probleme und Defizite der Integration naturschutzrelevanter Daten im Rahmen der Forsteinrichtung. Schr. f. Landschaftspfl. u. Natursch. 43:231-245.

Vanclay, J.K., 1994: Modelling forest growth and yield. CAB International, 312 S.

Vilčko, F. und Gadow, K. v., 2002: Konstruktion eines alterabhängigen Bestandesmodells für Fichte (Picea Abies L. Karst). Institut für Forsteinrichtung und Ertragskunde, GeorgAugust-Universität Göttingen, 13 S. (Unveröffentlichtes Manuskript).

Volin, C. und Buongiorno, J., 1996: Effects of alternative management regimes on forest stand structure, species composition and incombe: a model for the Italian Dolomites. Forest Ecology and Management 87: 107-125.

Ware, G. O. und Clutter, J. L., 1971: A mathematical programming system for the management of industrial forests. For. Sci. 17: 428-445.

Wikström, P., 2000: Solving stand-level planning problems that involve multiple criteria and a single-tree growth model. Doctoral Thesis. Swedish University of Agricultural Sciences. Umeå.

Xunta de Galicia, 1992: Plan forestal de Galicia. Síntesis. Consellería de Agricultura, Ganadería e Montes. 



\section{Veröffentlichung I}

Grundflächenmodelle für gleichaltrige Fichtenreinbestände

Eine vergleichende Analyse

GurJANOV, M., SÁNCHEZ OrOIS, S. UND SCHRÖDER, J.

Erschienen in:

Centralblatt für das gesamte Forstwesen. Jahrgang 2000, Heft 3/4:187-198. 



\title{
Grundflächenmodelle für gleichaltrige Fichtenreinbestände Eine vergleichende Analyse
}

Basal area models for even-aged Norway spruce stands

A comparative analysis

\author{
MichaIl GuRJANOv, SOFÍA SÁNCHEZ OrOIS UND JÖRG SCHRÖDER \\ Institut für Forsteinrichtung und Ertragskunde. Georg-August-Universität Göttingen \\ Büsgenweg 5, 37077 Göttingen. Germany
}

\begin{abstract}
Zusammenfassung
Ziel der vorliegenden Arbeit ist die vergleichende Analyse verschiedener Grundflächenmodelle für gleichaltrige Fichtenreinbestände (Picea abies L. Karst) in Norddeutschland. Die zur Parametrisierung der Modelle verwendeten Daten wurden von der NIEDERSÄCHSISCHEN FORSTLICHEN VERSUCHSANSTALT zur Verfügung gestellt. Die in dieser Arbeit untersuchten Modelle sind dem Ansatz der pfad-invarianten algebraischen Differerenzenform (PID-Typ) zuzuordnen und ermöglichen die Schätzung der Grundflächenentwicklung in Funktion von Bestandesvariablen wie z.B. der Oberhöhe und der Stammzahl. Die Güte der Anpassung wird zunächst mit Hilfe verschiedener statistischer Kriterien beurteilt. Um die Genauigkeit der Modellprognose zu überprüfen, wurde schließlich eine Validierung mit Hilfe eines unabhängigen Datensatzes durchgeführt. Bezüglich der untersuchten Kriterien zeigen die Modelle von Schumacher und Souter die besten Ergebnisse.
\end{abstract}

Schlagwörter: Grundfläche, Grundflächenmodellierung, Grundflächenentwicklung, forstlicher Eingriff, Fichtenbestände. 


\section{Einleitung}

Die Entwicklung von Modellen für die Prognose von Wachstum und Ertrag ist seit jeher das zentrale Thema der Waldwachstumsforschung. Wachstumssimulatoren werden heute nicht nur zur kurafristigen Prognose bei der Fortschreibung von gespeicherten Inventurdaten eingesetzt, sondern dienen vor allem auch zur Simulation unterschiedlicher Waldentwicklungspfade in realen oder hypothetischen Beständen (Rodríguez, 1995; Gadow u. Hui, 1999).

Ein wichtiges Element von Bestandeswuchssimulatoren sind Modelle, mit denen die Entwicklung der Bestandesgrundfläche prognostiziert werden kann. Die Bestandesgrundfläche ist ein wichtiges Dichtemaß, das sowohl die Dimension der Bäume als auch die Stammzahl berücksichtigt und u.a. zur Analyse der Beziehung zwischen Bestockungsgrad und Volumenzuwachs verwendet werden kann (vgl. Assmann, 1961). Außerdem ist die Grundfläche in Kombination mit der Stammzahl geeignet, die Art und Stärke von Durchforstungseingriffen zu beschreiben und zu definieren (z.B. Álvarez González, 1997; Gadow und Hui, 1999; Staupendahl, 1999).

Abbildung 1 veranschaulicht die zwei grundsätzlichen Prozesse durch die das Grundflächenwachstum beeinflusst wird, nämlich einerseits das natürliche Wachstum zwischen zwei Eingriffen und andererseits die eingriffsbedingte Veränderung.

Bestandesgrundflächenmodelle lassen sich in zwei unterschiedliche Gruppen unterteilen:

- Pfad-invariante algebraische Differenzenform Modelle (PID-Typ), die die Grundfläche im Alter $\left(A_{2}\right)$ als Funktion von Grundfläche, Höhe und Stammzahl im Alter $\left(A_{1}\right)$ und Höhe und Stammzahl im Alter ( $\left.\mathrm{A}_{2}\right)$ bestimmen (z.B. Schumacher, 1939; Souter, 1986; Forss, 1994).

- Differentialgleichungen zur Schätzung des jährlichen Grundflächenzuwachses (DIFTyp). Beispiele sind die auf dem state space approach basierenden Modell von García (1994), Rodríguez Soalleiro (1995) und Kvist Johannsen (1999). 


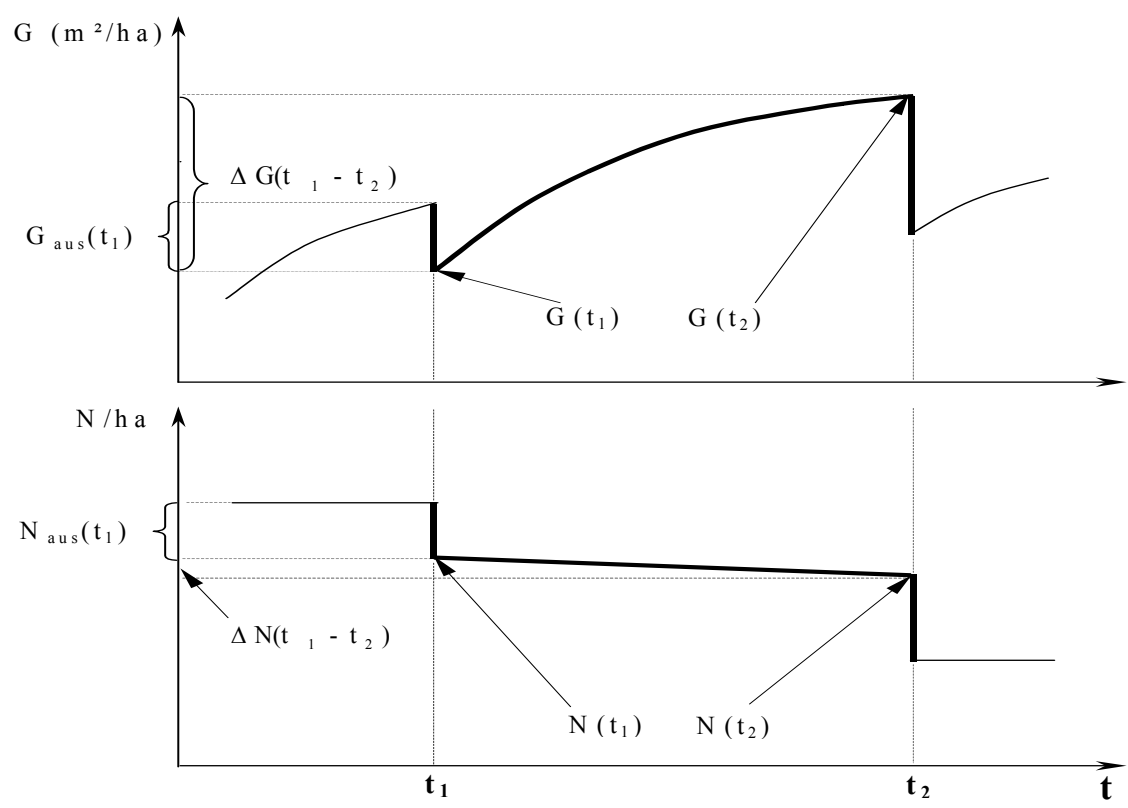

Abb.1. Schematische Abfolge von Wachstum und Eingriff $\left(G=\right.$ Grundfläche $\left(\mathrm{m}^{2} / \mathrm{ha}\right), G_{\text {aus }}=$ bei Durchforstung ausscheidende Grundfläche $\left(\mathrm{m}^{2} / \mathrm{ha}\right), G\left(t_{1}\right)$ und $G\left(t_{2}\right)=$ Grundfläche im Alter $t_{1}$ und $t_{2}\left(m^{2} / h a\right), \Delta G\left(t_{1}-t_{2}\right)=$ Zunabme der Grundfläche durch Wachstum $\left(m^{2} / h a\right), N=$ Stammzabl pro ha, $N_{\text {aus }}=$ bei Durchforstung ausscheidende Stammzabl pro ha, $N\left(t_{1}\right)$ und $N\left(t_{2}\right)=$ Stammzabl pro ha im Alter $t_{1}$ und $t_{2}, \Delta N\left(t_{1}-t_{2}\right)=$ Abnahme der Stammzabl pro ha durch natürliche Mortalität, wobei bei hoher Intensität der Forstwirtschaft und kurzen Zeitperioden zwischen den Aufnabmen, $\Delta N\left(t_{1}-t_{2}\right) \approx 0$ ist.

Fig. 1. Schematic sequence of growth and intervention. $\left(G=\right.$ basal area $\left(\mathrm{m}^{2} / \mathrm{ha}\right), G_{\text {aus }}=$ thinned basal area $\left(m^{2} / h a\right), G\left(t_{1}\right)$ und $G\left(t_{2}\right)=$ basal area at age $t_{1}$ and $t_{2}\left(m^{2} / h a\right), \Delta G\left(t_{1}-t_{2}\right)=$ basal area increment $\left(\mathrm{m}^{2} / \mathrm{ha}\right), N=$ stem number per ha, $N_{\text {aus }}=$ removed stem number per $h a, N\left(t_{1}\right)$ und $N\left(t_{2}\right)=$ stem number per ha at age $t_{1}$ and $t_{2}, \Delta \mathrm{N}\left(t_{1}-t_{2}\right)=$ removed stem number per ha due to natural mortality. If the thinning intensity is high and the time period between inventories short, then $\Delta N\left(t_{1}-t_{2}\right) \approx 0$.

Ziel der vorliegenden Arbeit ist es, die Eignung verschiedener PID Modelle für durchforstete, gleichaltrige Fichtenbestände zu vergleichen. Im Einzelnen sollen dabei die Ansätze von Schumacher (1939), Pienaar und Shiver (1986), Souter (1986), Hui und Gadow (1993, 1999) sowie Forss (1994) untersucht werden.

\section{Daten}

Zur Parametrisierung der zu analysierenden Modelle wurden die Daten von 19 Fichtenversuchsflächen verwendet ${ }^{1}$. Eine Beschreibung der Versuchsflächen und der aufgenommenen Daten findet sich in Tabelle 1.

\footnotetext{
${ }^{1}$ Die von Schübeler (1997) aufbereiteten Daten wurden freundlicherweise von der Niedersächsischen Forstlichen Versuchsanstalt zur Verfügung gestellt.
} 


\begin{tabular}{|c|c|c|c|c|c|c|}
\hline \multirow{2}{*}{ Forstamt } & \multirow{2}{*}{$\begin{array}{c}\text { Df.-Vfl. } \\
\text { Nr. }\end{array}$} & \multirow{2}{*}{ Abt. } & \multicolumn{2}{|c|}{ Beobachtungsperiode } & \multirow{2}{*}{$\begin{array}{c}\text { Anzahl } \\
\text { Aufnahmen/ } \\
\text { Eingriffe }\end{array}$} & \multirow{2}{*}{ Zielsetzung } \\
\hline & & & Anfangsalter & Endalter & & \\
\hline Paderborn & $64 / 1$ & 90 & 23 & 89 & $18 / 13$ & Schnellwuchs \\
\hline Paderborn & $64 / 2$ & 90 & 23 & 89 & $18 / 16$ & schwache Niederdurchforstung \\
\hline Paderborn & $64 / 3$ & 90 & 28 & 89 & $17 / 16$ & mäßige Niederdurchforstung \\
\hline Monschau & $14 / 1$ & 314 & 26 & 91 & $13 / 11$ & Schnellwuchs \\
\hline Monschau & $14 / 2$ & 314 & 26 & 91 & $13 / 12$ & schwache Niederdurchforstung \\
\hline Morbach & $54 / 1$ & 150 & 32 & 110 & $17 / 15$ & Schnellwuchs \\
\hline Morbach & $54 / 2$ & 150 & 32 & 110 & $17 / 17$ & schwache Niederdurchforstung \\
\hline Morbach & $54 / 3$ & 150 & 54 & 110 & $14 / 12$ & mäßige Niederdurchforstung \\
\hline Westerhof & $75 / 3$ & 140 & 38 & 103 & $18 / 17$ & schwache Niederdurchforstung \\
\hline Westerhof & $75 / 5$ & 140 & 38 & 81 & $12 / 11$ & schwache Niederdurchforstung \\
\hline Westerhof & $75 / 6$ & 140 & 60 & 103 & $13 / 11$ & starke Niederdurchforstung \\
\hline Elbrighausen & $55-57 / 1$ & 55 & 60 & 124 & $12 / 11$ & Plenterdurchforstung \\
\hline Elbrighausen & $55-57 / 2$ & 57 & 60 & 124 & $12 / 11$ & starke Niederdurchforstung \\
\hline Neuenheerse & $83 / 3$ & 240 & 79 & 128 & $16 / 11$ & mäßige Niederdurchforstung \\
\hline Neuenheerse & $83 / 6$ & 240 & 79 & 128 & $16 / 12$ & starke Niederdurchforstung \\
\hline Altenau & 45 & 45 & 53 & 128 & $13 / 8$ & starke Lichtung \\
\hline Westerhof & $78 / 1$ & 78 & 36 & 106 & $15 / 12$ & starke Niederdurchforstung \\
\hline Westerhof & $78 / 2$ & 78 & 36 & 106 & $15 / 13$ & mäßige Niederdurchforstung \\
\hline Oderhaus & 62 & 49 & 78 & 139 & $15 / 12$ & mäßige Niederdurchforstung \\
\hline
\end{tabular}

Tab. 1. Kurze Beschreibung der Versuchsflächen (Daten aus Schübeler, 1997).

Tab. 1. Short description of the experimental plots (data from Schübeler, 1997).

Insgesamt stehen 228 Beobachtungsintervalle für die Bestandesvariablen Alter, Oberhöhe, Stammzahl und Grundfläche zwischen zwei Aufnahmen bzw. forstlichen Eingriffen zur Verfügung. In Abbildung 2 wird die Entwicklung der Oberhöhen, der Stammzahlen und der Grundflächen über dem Bestandesalter, sowie die Grundflächenentwicklung über der Oberhöhenentwicklung dargestellt.

Wie aus der Oberhöhenentwicklung in Abb.2(a) zu ersehen ist, lassen sich die Daten nach unterschiedlichen Standortbonitäten unterteilen. Außerdem zeigt die Stammzahlentwicklung in Abb.2(c), dass die natürliche Mortalität wegen der geringen Zeitperiode zwischen zwei forstlichen Eingriffen keine Rolle spielt. 

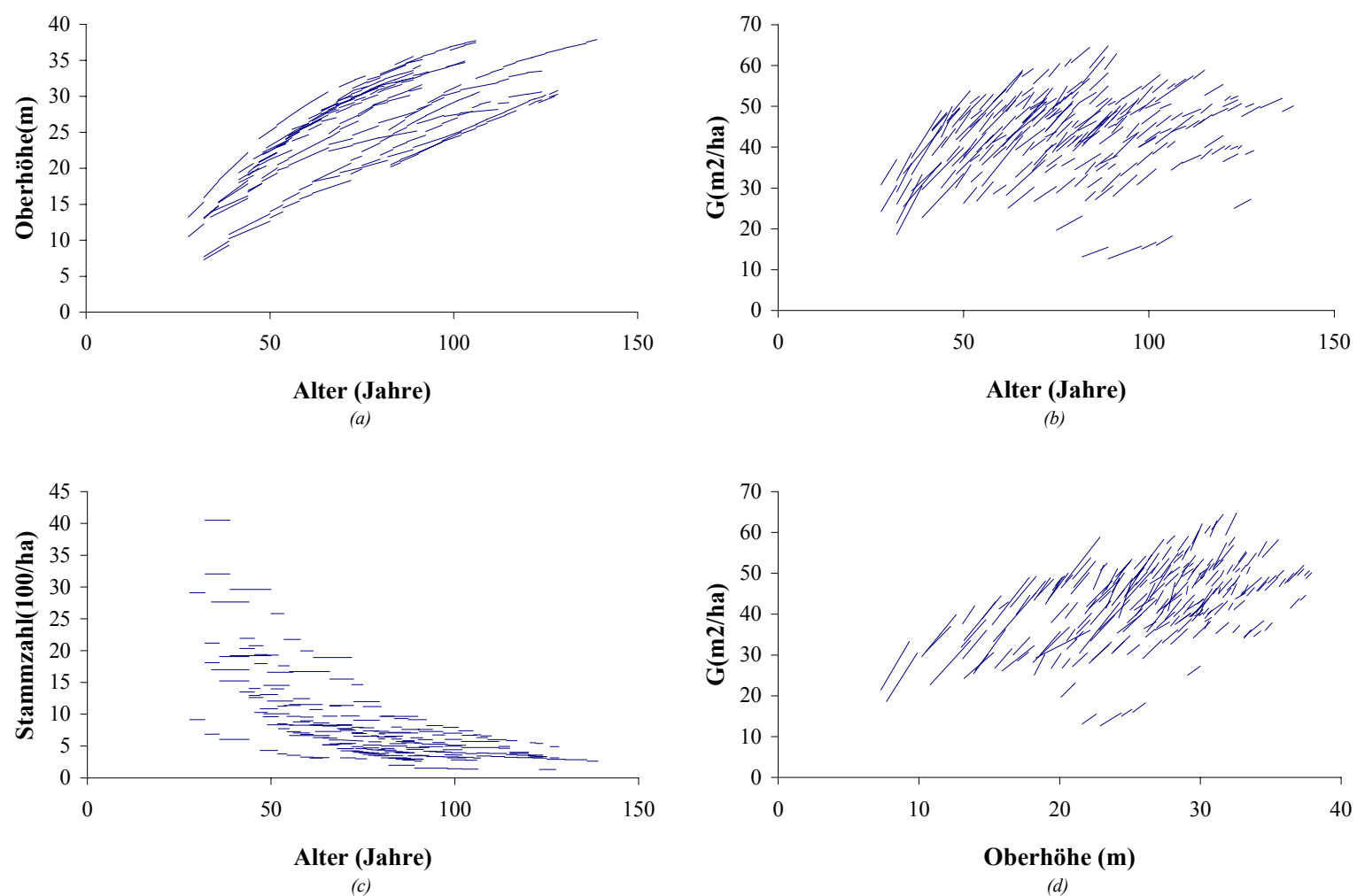

Abb. 2. Graphische Darstellung der von den 19 Versuchsflächen erbobenen Daten: Oberböhenentwicklung (a), Grundflächenentwicklung (b), Stammaablentwicklung (c) und Grundflächen- über Oberböhenentwicklung (d).

Fig. 2. Graphic presentation of the data from the 19 experimental plots. Height development (a), basal area development (b), stem number development (c) and basal area development versus height development (d).

\section{Grundflächenmodelle}

Wie bereits erwähnt, konzentriert sich die Arbeit auf die vergleichende Analyse von Grundflächenmodellen, die dem PID-Typ zuzuordnen sind. Der Vorteil dieses Ansatzes liegt unter anderem darin, dass bei der Anpassung der Modelle auch solche Änderungsraten verwendet werden können, die sich auf unterschiedlich lange Zeitintervalle beziehen.

Das von Pienaar und Shiver entwickelte Wuchsmodell ermöglicht eine Prognose der Grundflächenentwicklung in Funktion von Alter, Höhe und Stammzahl (Pienaar u. Shiver, 1986):

$$
\ln G_{2}=\ln G_{1}+\alpha \cdot\left(\frac{1}{A_{2}}-\frac{1}{A_{1}}\right)+\beta \cdot\left(\ln N_{2}-\ln N_{1}\right)+\delta \cdot\left(\ln H_{2}-\ln H_{1}\right)+\varepsilon \cdot\left(\frac{\ln H_{2}}{A_{2}}-\frac{\ln H_{1}}{A_{1}}\right)
$$


Wobei $\mathrm{A}_{1}$ und $\mathrm{A}_{2} \quad=$ Alter am Anfang und am Ende der Zeitperiode zwischen forstlichen Eingriffen;

$G_{1}$ und $G_{2}=$ Grundfläche im Alter $A_{1}$ und $A_{2},\left(m^{2} / h a\right)$;

$\mathrm{H}_{1}$ und $\mathrm{H}_{2} \quad=$ Oberhöhe im Alter $\mathrm{A}_{1}$ und $\mathrm{A}_{2},(\mathrm{~m})$;

$\mathrm{N}_{1}$ und $\mathrm{N}_{2} \quad=$ Stammzahl im Alter $\mathrm{A}_{1}$ und $\mathrm{A}_{2}$;

$\alpha, \beta, \delta$ und $\varepsilon=$ Modellparameter.

Gleichung (1) wurde von Forss (1994) wie folgt vereinfacht:

$$
\ln G_{2}=\ln G_{1}+\alpha \cdot\left(\frac{1}{A_{2}}-\frac{1}{A_{1}}\right)+\beta \cdot\left(\ln H_{2}-\ln H_{1}\right)+\delta \cdot\left(\ln N_{2}-\ln N_{1}\right)
$$

Bei starker Durchforstung und einer geringer Zeitperiode zwischen zwei forstlichen Eingriffen approximiert die Stammzahl im Alter $A_{2}$ die Stammzahl im Alter $A_{1}$, also $\mathrm{N}_{1} \approx \mathrm{N}_{2}$ (vgl. Abb.1 und Abb. 2c). In diesem Fall kann Gl. 2 durch Gl. 3 ersetzt werden:

$$
\ln G_{2}=\ln G_{1}+\alpha \cdot\left(\frac{1}{A_{2}}-\frac{1}{A_{1}}\right)+\beta \cdot\left(\ln H_{2}-\ln H_{1}\right)
$$

Das Modell von Hui und Gadow (1993) hat sich bei der Baumart Cunninghamia lanceolata gut bewährt. Die Gleichung hat 3 Parameter und ermöglicht die Fortschreibung einer tatsächlich ermittelten Bestandesgrundfläche:

$$
G_{2}=G_{1} \cdot N_{2}^{1-\alpha \cdot H_{2}^{\beta}} \cdot N_{1}^{\alpha \cdot H_{1}^{\beta}-1} \cdot\left(\frac{H_{2}}{H_{1}}\right)^{\delta}
$$

Unter der oben genannten Bedingung $\left(\mathrm{N}_{1} \approx \mathrm{N}_{2}\right)$ kann man Gl. 4 auch darstellen als:

$$
G_{2}=G_{1} \cdot\left(\frac{H_{2}}{H_{1}}\right)^{\delta}
$$

Schumacher $(1939,1960)$ hat ein altersabhängiges Grundflächenmodell vorgeschlagen, dass später auch von Clutter (1963) sowie Sullivan und Clutter (1972) verwendet wurde:

$$
\ln G_{2}=\alpha+\left(\ln G_{1}-\alpha\right) \cdot \frac{A_{1}}{A_{2}}
$$


Souter (1986) hat auf der Basis von Schumachers Modell die folgende Gleichung entwickelt:

$$
\ln G_{2}=\left(\frac{A_{1}}{A_{2}}\right) \cdot \ln G_{1}+\alpha \cdot\left(1-\frac{A_{1}}{A_{2}}\right)+\beta \cdot S \cdot\left(1-\frac{A_{1}}{A_{2}}\right)+\gamma \cdot\left(\ln N_{2}-\left(\frac{A_{1}}{A_{2}}\right) \cdot \ln N_{1}\right)
$$

wobei $\mathrm{A}_{1}$ und $\mathrm{A}_{2} \quad=$ Alter am Anfang und am Ende der Zeitperiode zwischen forstlichen Eingriffen;

$\mathrm{G}_{1}$ und $\mathrm{G}_{2}=$ Grundfläche im Alter $\mathrm{A}_{1}$ und $\mathrm{A}_{2},\left(\mathrm{~m}^{2} / \mathrm{ha}\right)$;

$\mathrm{S}=$ Standortbonität;

$\mathrm{N}_{1}$ und $\mathrm{N}_{2} \quad=$ Stammzahl im Alter $\mathrm{A}_{1}$ und $\mathrm{A}_{2}$;

$\alpha, \beta$ und $\gamma \quad=$ Modellparameter.

Gleichung (8) ist eine Vereinfachung des von Souter (1986) vorgeschlagenen Modells:

$$
\ln G_{2}=\left(\frac{A_{1}}{A_{2}}\right) \cdot \ln G_{1}+\alpha \cdot\left(1-\frac{A_{1}}{A_{2}}\right)+\beta \cdot\left(\ln N_{2}-\left(\frac{A_{1}}{A_{2}}\right) \cdot \ln N_{1}\right)
$$

\section{Analyse und Ergebnisse}

Zunächst wurden die Parameter der Gleichungen (1-6) und (8) mit Hilfe der Programmpakete STATISTICA und SAS unter Verwendung linearer oder nichtlinearer Regression auf der Basis der verfügbaren Daten berechnet. Tabelle 2 zeigt die für die einzelnen Modelle ermittelten Parameter.

\begin{tabular}{|l|c|c|c|c|c|}
\hline \multicolumn{2}{|c|}{ Modell } & \multicolumn{4}{c|}{ Geschätzte Parameter } \\
\hline Autor & Gl. Nr. & $\alpha$ & $\beta$ & $\delta$ & $\varepsilon$ \\
\hline Pinaar u. Shiver & $(1)$ & 33,62 & 0,004 & 2,51 & $-1,05$ \\
Forss & $(2)$ & 6,71 & 2,080 & 0,0001 & \\
Forss & $(3)$ & 6,71 & 2,080 & & \\
Hui u. Gadow & $(4)$ & 0,70 & 0,110 & 2,66 & \\
Hui & $(5)$ & & & 1,89 & \\
Schumacher & $(6)$ & 5,09 & & & \\
Souter & $(8)$ & 5,25 & $-0,020$ & & \\
\hline
\end{tabular}

Tab. 2. Geschät_te Parameterwerte für die Grundflächenmodelle (1-6) und (8).

Tab. 2. Estimated parameters obtained for the basal area models (1-6) and (8).

Bei der Evaluierung der Modelle wurde nach Schätzgenauigkeit (Präzision) und gerichteter Verzerrung (Bias) unterschieden. Zur Beurteilung der Schätzgenauigkeit wurde der Koeffizient der Korrelation zwischen den geschätzten $(\hat{y})$ und beobachteten $(y)$ Werten $\left(R_{y \hat{y}}\right)$ sowie die Wurzel des mittleren quadratischen Fehlers (RMSE) verwendet. Das mittlere Residuum (MRES) ist ein Maß für die durchschnittliche gerichtete Abweichung. Tabelle 3 
zeigt die Formeln zur Berechnung der Kriterien RMSE und MRES. Ein Beispiel für die Anwendung der genannten Kriterien findet sich bei Trincado und Gadow (1996). Die in dieser Arbeit für die einzelnen Modelle ermittelten Werte sind in Tabelle 4 aufgelistet.

\begin{tabular}{|l|c|c|}
\hline \multicolumn{1}{|c|}{ Kriterien } & Formel & Idealer Wert \\
\hline MRES (mittleres Residuum) & $\frac{\sum\left(X_{i}-\hat{X}_{i}\right)}{n}$ & 0 \\
\hline RMSE (Wurzel des mitt. quad. Fehlers) & $\sqrt{\frac{\sum\left(X_{i}-\hat{X}_{i}\right)^{2}}{n-p-1}}$ & 0 \\
\hline
\end{tabular}

Tab. 3. Kriterien zur Evaluierung der Modelle $\left(\hat{X}_{i}=\right.$ geschätzter Wert; $X_{i}=$ beobachteter Wert; $n=$ Anzabl der Beobachtungen; $p=$ Anzabl der Funktionsparameter).

Tab. 3. Criteria for evaluating the models $\left(\hat{X}_{i}=\right.$ predicted value; $X_{i}=$ observed value; $n=$ number of measurements; $p=$ number of model parameters).

Die Modelle wurden schließlich mit Hilfe eines unabhängigen Datensatzes validiert. Dieser Datensatz stammt ebenfalls von den Versuchsflächen aus dem Solling in Niedersachsen. Zunächst wurde mit Hilfe der einzelnen Modelle die Grundflächen (Gl. 4 u. 5) bzw. die Logarithmen der Grundflächen (Gl. 1, 2, 3, 6 u. 8) im Alter t2 geschätzt. Zur Beurteilung der Schätzgenauigkeit wurden erneut die oben genannten Kriterien verwendet. Weil die Modelle von Hui und Gadow (Gl. 4) und Hui (Gl. 5) die Grundflächen statt die Logarithmen der Grundflächen schätzen, war es nicht möglich, die bei der Validierung erhaltenen Werte für die Kriterien MRES und RMSE direkt zu vergleichen. Daher wurden die Relativwerte der Residuen verwendet, d.h. die Residuen der Modelle wurden durch die geschätzten Werte geteilt.

\begin{tabular}{|c|c|c|c|c|c|c|c|}
\hline Kriterien & Pienaar & Forss(G1.2) & Forss(G1.3) & Hui(G1.4) & Hui(G1.5) & Schumacher & Souter \\
\hline $\mathrm{R}_{\mathrm{y} \hat{\mathrm{y}}}$ & 99,2 & 99,0 & 99,0 & 98,9 & 98,9 & 99,4 & 99,4 \\
\hline MRES & 0,0016 & 0,0036 & 0,0036 & 0,0131 & 0,0143 & 0,0010 & 0,0009 \\
\hline RMSE & 0,0006 & 0,0006 & 0,0006 & 0,0023 & 0,0024 & 0,0005 & 0,0005 \\
\hline
\end{tabular}

Tab. 4. Kriterien zur Analyse der Modellgenanigkeit: $R_{y \hat{y}}=$ Korrelationskoeffizient; $M R E S=$ mittleres Residuum; RMSE = Wurzel des mittleren quadratischen Feblers.

Tab. 4. Criteria for analysing the model accuracy: $R_{y y}=$ coefficient of correlation; $M R E S=$ mean residual; RMSE = root mean square error. 
Tabelle 4 zeigt, dass es zwischen den einzelnen Modellen lediglich geringe Unterschiede in der Genauigkeit der Anpassung gibt. Die Werte des Korrelationskoeffizienten $\left(\mathrm{R}_{\mathrm{yy}}\right)$ variieren zwischen 98,9 und 99,4. Die besten Ergebnisse bezüglich der Kriterien MRES und RMSE zeigen die Modelle von Schumacher und Souter.

In Tabelle 5 und Tabelle 6 sind die für die einzelnen Modelle berechneten MRESWerte und RMSE-Werte stratifiziert nach Altersklassen aufgelistet. Abbildung 3 veranschaulicht diese Ergebnisse graphisch. Analog zu Tabelle 4 zeigt Tabelle 7 die Ergebnisse für das gesamte Datenmaterial.

\begin{tabular}{|c|c|c|c|c|c|c|c|}
\cline { 2 - 8 } \multicolumn{1}{c|}{} & \multicolumn{7}{c|}{ MRES } \\
\hline Alter & Pienaar & Forss(Gl.2) & Forss(Gl.3) & Schumacher & Souter & Hu(Gl.4)i & Hui(Gl.5) \\
\hline $40-50$ & 0,0015 & 0,0016 & 0,0009 & $-0,0001$ & $-0,0001$ & 0,0032 & 0,0031 \\
\hline $50-60$ & 0,0010 & 0,0020 & 0,0007 & 0,0002 & 0,0002 & 0,0027 & 0,0026 \\
\hline $60-70$ & 0,0066 & 0,0053 & 0,0052 & 0,0004 & 0,0004 & 0,0193 & 0,0194 \\
\hline $70-80$ & 0,0033 & 0,0032 & 0,0026 & $-0,0002$ & $-0,0002$ & 0,0093 & 0,0093 \\
\hline $80-90$ & 0,0039 & 0,0042 & 0,0033 & 0,0000 & $-0,0001$ & 0,0109 & 0,0110 \\
\hline $90-100$ & 0,0022 & 0,0037 & 0,0019 & 0,0003 & 0,0003 & 0,0066 & 0,0070 \\
\hline $100-110$ & 0,0027 & 0,0036 & 0,0025 & 0,0006 & 0,0006 & 0,0085 & 0,0088 \\
\hline $110-120$ & 0,0016 & 0,0031 & 0,0015 & 0,0003 & 0,0002 & 0,0049 & 0,0053 \\
\hline $120-130$ & 0,0014 & 0,0024 & 0,0014 & 0,0004 & 0,0004 & 0,0042 & 0,0046 \\
\hline
\end{tabular}

Tab. 5. Nach Altersklassen stratifizierte MRES-Werte.

Tab. 5. MRES-values according to age-classes.

\begin{tabular}{|c|c|c|c|c|c|c|c|}
\cline { 2 - 8 } \multicolumn{1}{c|}{} & \multicolumn{7}{c|}{ RMSE } \\
\hline Alter & Pienaar & Forss(Gl.2) & Forss(Gl.3) & Schumacher & Souter & Hui(Gl.4) & Hui(Gl.5) \\
\hline $40-50$ & 0,0073 & 0,0078 & 0,0046 & 0,0006 & 0,0006 & 0,0155 & 0,0151 \\
\hline $50-60$ & 0,0051 & 0,0099 & 0,0034 & 0,0014 & 0,0015 & 0,0130 & 0,0125 \\
\hline $60-70$ & 0,0228 & 0,0162 & 0,0176 & 0,0032 & 0,0033 & 0,0656 & 0,0649 \\
\hline $70-80$ & 0,0107 & 0,0090 & 0,0085 & 0,0010 & 0,0010 & 0,0300 & 0,0296 \\
\hline $80-90$ & 0,0137 & 0,0133 & 0,0116 & 0,0023 & 0,0024 & 0,0364 & 0,0360 \\
\hline $90-100$ & 0,0071 & 0,0133 & 0,0064 & 0,0017 & 0,0018 & 0,0207 & 0,0217 \\
\hline $100-110$ & 0,0090 & 0,0116 & 0,0080 & 0,0025 & 0,0024 & 0,0274 & 0,0276 \\
\hline $110-120$ & 0,0066 & 0,0113 & 0,0060 & 0,0018 & 0,0018 & 0,0189 & 0,0198 \\
\hline $120-130$ & 0,0053 & 0,0086 & 0,0051 & 0,0015 & 0,0014 & 0,0157 & 0,0164 \\
\hline
\end{tabular}

Tab. 6. Nach Altersklassen stratifizierte RMSE-Werte.

Tab. 6. RMSE-values according to age-classes. 


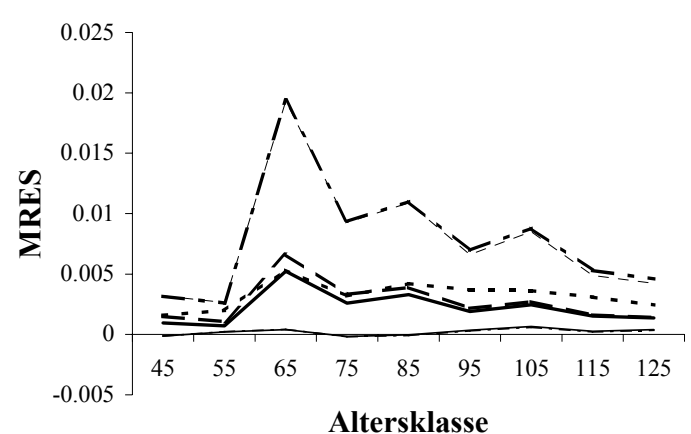

(a)

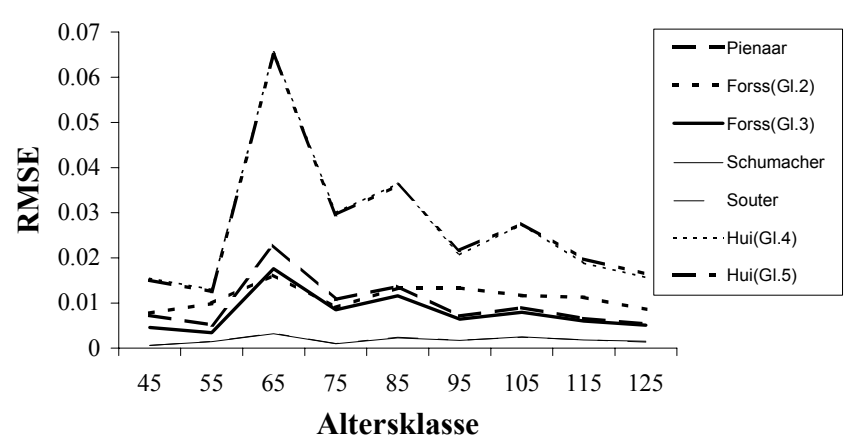

(b)

Abb. 3. Nach Altersklassen stratifizierte Werte für MRES (a) und RMSE (b).

Fig. 3. MRES (a) and RMSE (b) according to age-classes.

Wie aus den Abbildungen $3 \mathrm{a}$ und $3 \mathrm{~b}$ zu entnehmen ist, zeigen die Modelle von Schumacher (Gl. 6) und Souter (Gl. 8) die kleinsten, die Modelle von Hui und Gadow (Gl. 4) sowie Hui (Gl. 5) die größten Werte für MRES und RMSE. Für die Altersklassen 60-70 und 100-110 wurden jeweils die größten Werte ermittelt.

\begin{tabular}{|c|c|c|c|c|c|c|c|}
\hline Kriterien & Pienaar & Forss(G1.2) & Forss(G1.3) & Hui(G1.4) & Hui(G1.5) & Schumacher & Souter \\
\hline $\mathrm{R}_{\mathrm{y} \hat{y}}$ & 98.5 & 99.4 & 99.1 & 98.9 & 99.0 & 99.9 & 99.9 \\
\hline MRES & 0,0230 & 0,0278 & 0,0192 & 0,0646 & 0,0656 & 0,0021 & 0,0186 \\
\hline RMSE & 0,0038 & 0,0040 & 0,0031 & 0,0106 & 0,0107 & 0,0007 & 0,0002 \\
\hline
\end{tabular}

Tab. 7. Kriterien zur Analyse der Modellgenanigkeit: $R_{y y}=$ Korrelationskoeffizient; MRES= mittleres Residuum; RMSE = Wurzel des mittleren quadratischen Fehlers.

Tab. 7. Criteria for evaluating the model accuracy: $R_{y y}=$ coefficient of correlation, $M R E S=$ mean residual; $\mathrm{RMSE}=$ root mean square error.

Um schließlich die Schätzgenauigkeit für die Modelle von Schumacher und Souter graphisch vergleichen und überprüfen zu können, werden in Abbildung 4 für beide Datensätze jeweils die geschätzten über den beobachteten Werten aufgetragen. 
Schumacher

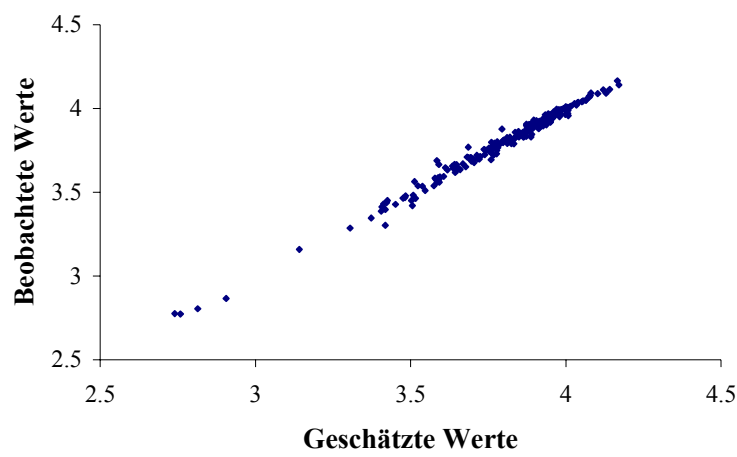

(a)

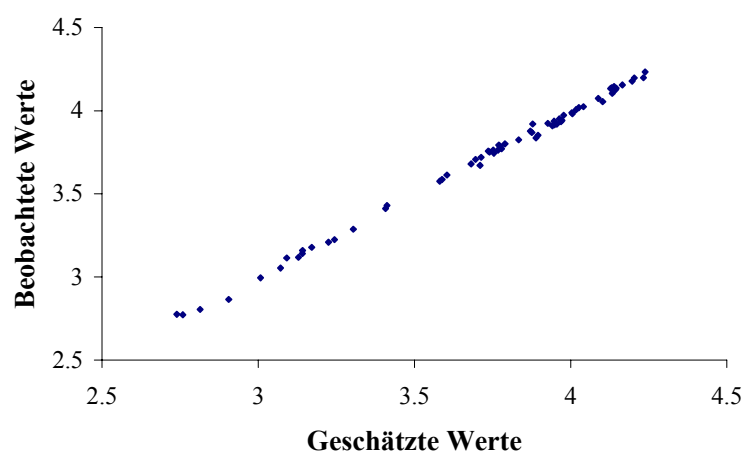

(c)

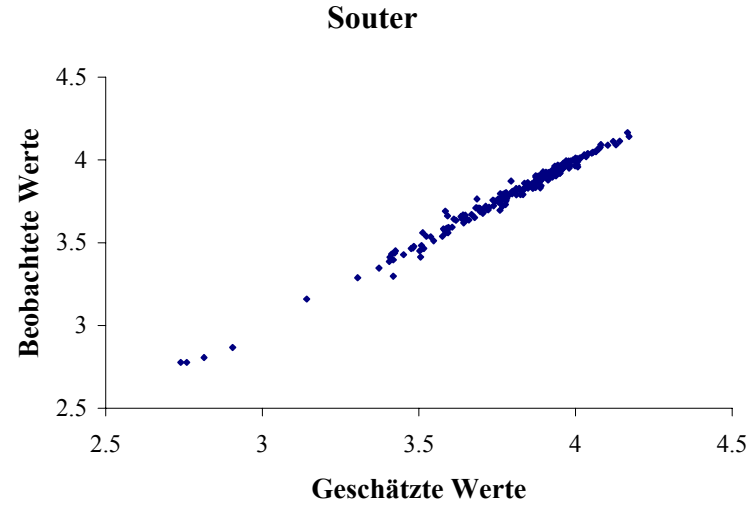

(b)

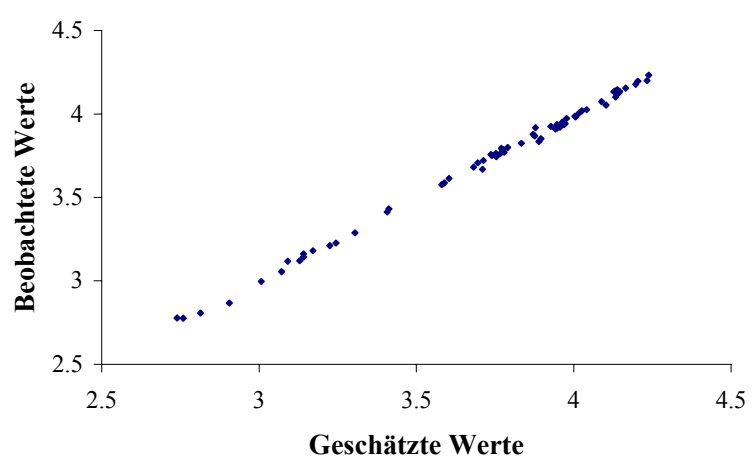

(d)

Abb. 4. Nach den Modellen von Schumacher und Souter geschätzte Werte über den beobachteten Werten. (a) und (b) beriehen sich auf den ersten, (b) und (c) auf den zweiten (unabbängigen) Datensat:

Fig. 4. Predicted versus observed values according to Schumacher's and Souter's model. (a) and (b) refer to the first, (b) and (c) to the second (independent) data set.

\section{Diskussion und Schlussfolgerungen}

Alle in dieser Arbeit analysierten Grundflächenmodelle zeigen geringe RMSE-Werte sowie hohe Korrelationskoeffizienten für die Korrelation zwischen den geschätzten und beobachteten Werten. Allerdings deutet dieses Ergebnis nicht generell darauf hin, dass alle Modelle akkurate Schätzungen der Grundflächenentwicklung ermöglichen. So zeigte sich bei der Validierung der Modelle mit Hilfe des unabhängigen Datensatzes, dass insbesondere die Gleichungen (4) und (5) erhebliche systematische Verzerrungen aufweisen (vgl. Tab. 5, Abb.3b). Nur die Modelle von Schumacher und Souter (Gl. 6 u. 8) zeigen diesbezüglich zufriedenstellende Ergebnisse.

Wie die graphische Darstellung der geschätzten über den beobachteten Werten in Abbildung 4 zeigt, sind die Unterschiede zwischen den beiden Modellen für beide Datensätze unerheblich. Daher können generell beide Modelle zur Schätzung des Grundflächenwachstums empfohlen werden. Allerdings hat das Modell von Schumacher den 
praktischen Vorteil, dass die Schätzung mit Hilfe von lediglich zwei unabhängigen Variablen erfolgt.

Da die Änderungsraten der verwendeten Datensätze auf unterschiedliche Zeitintervalle und Standortbonität bezogen sind, konnten in dieser Arbeit keine Modelle des DIF-Typs getestet werden (s.o.). In einer zukünftigen Untersuchung wäre es daher interessant, diesen Modelltyp ebenfalls zu berücksichtigen und mit den in dieser Arbeit analysierten Ansätzen zu vergleichen.

\section{Danksagung}

Die Daten für diese Untersuchung wurden uns in großzügiger Weise von der NiEDERSÄCHSISCHEN FORSTLICHEN VersuCHSANSTALT zur Verfügung gestellt. Dafür bedanken wir uns sehr herzlich. Für methodische Unterstützung und zahlreiche Hinweise danken wir Herrn Prof. Dr. Klaus v. Gadow ${ }^{2}$, Herrn Prof. Dr. Pedro Real ${ }^{3}$, Herrn Prof. Dr. Joachim Saborowski ${ }^{4}$, Herrn Dr. Juha Lappi ${ }^{5}$ sowie den Mitarbeitern im Institut für Forsteinrichtung und Ertragskunde, insbesondere Herrn Diplom-Forstwirt Kai Staupendahl.

\section{Summary}

The purpose of this paper is to compare different stand level models for predicting basal area growth of even-aged stands of Norway spruce (Picea abies L. Karst.) in Northern Germany. The data used to fit the models were provided by the NIEDERSÄCHSISCHE FORSTLICHE VERSUCHSANSTALT. All models tested in this paper belong to the path invariant algebraic difference form and can be used to predict basal area growth as a function of whole stand variables such as dominant stand height and number of stems per hectare. First, the performance of the models was tested using different statistical criteria. Finally, the models were validated using an independent data set. The models developed by Schumacher and Souter showed the best perfomance.

Keywords: Basal area, basal area modelling, basal area development, Norway spruce stands, thinning.

\footnotetext{
${ }^{2}$ Leiter des Instituts für Forsteinrichtung und Ertragskunde. Georg-August Universität Göttingen.

${ }^{3}$ Gastprofessor aus Concepción, Chile, zur Zeit am Institut für Forsteinrichtung und Ertragskunde tätig.

${ }^{4}$ Institut für forstliche Biometrie und Informatik. Georg-August Universität Göttingen.

${ }^{5}$ METLA, Finnland.
} 


\section{Literatur}

Álvarez, J.G., 1997: Análisis y caracterización de las distribuciones diamétricas de Pinus pinaster Ait. en Galicia. Diss. Uni. Madrid, 270 S.

Assman, E., 1961: Waldertragskunde. BLV Verlagsgesellschaft, München-Bonn-Wien, 490 S.

Clutter, J.L., 1963:Compatible growth and yield models for loblolly pine. For. Sci. 9:354-371.

Forss, E., 1994: Das Wachstum der Baumart Acacia mangium in Südkalimantan, Indonesien. Magister Diss., Fac. Of Forestry, Univ. of Göttingen, 87 S.

Gadow, K.v. und Hui, G. Y., 1993: Stammzahlentwicklung und potentielle Bestandesdichte bei Cunninghamia lanceolata. Centrallblat für das gesamte Forstwesen 110 (2): 41-48.

Gadow, K. v. und Hui, G. Y., 1999: Modelling forest development. Kluwer, 213 S.

García, O., 1994: The state space approach in growth modelling. Can. J. For. Res. 24: 18941903.

Hui, G. Y. und Gadow K.v., 1993: Zur Modellierung der Bestandesgrundflächenentwicklung - dargestellt am Beispiel der Baumart Cunninghamia lanceolata. Allgemeine Forst- und Jagdzeitung 164: 144-149.

Kvist Johannsen, V., 1999: A growth model for oak in Denmark. Ph. D. Dissertation, Danish Forest and Landscape Research Institute, Hørsholm, Denmark, 197 S.

Pienaar, L.V., und Shiver, B. D., 1986: Basal area prediction and projection equations for pine plantations. Forest Science 32(3):626-633.

Rodríguez, R. 1995: Crecimiento y producción de masas forestales regulares de Pinus pinaster Ait. en Galicia. Alternativas selvícolas posibles. Ph. D. Dissertation, Escuela Técnica Superior de Ingenieros de Montes. Madrid, 297 S.

Schübeler, D., 1997: Untersuchungen zur standortabhängigen Wachstumsmodellierung bei der Fichte. Dissertation zur Erlangung des Doktorgrades der Forstlichen Fakultät der Georg-August-Universität in Göttingen, 155 S.

Schumacher, F.X., 1939: A new growth curve and its applications to timber-yield studies. Journal of Forestry 37:819-820.

Schumacher, F.X., und Collie T.S., 1960: Growth and yield of natural stands of the southern pines. T. S. Collie Inc., Durham, N.C.

Souter, R. A., 1986: Dynamic stand structure in thinned stands of naturally regenerated loblolly pine in the Gerogia Piedmont. Ph. D. Thesis. University of Georgia, Athens, GA.

Staupendahl, K., 1999: Modelling thinnings based on the ratio of relative removal rates. Growth and yield modelling of tree plantations in south and east Africa. University of Joensuu, S. 183.

Sullivan, A.D., und Clutter, J. L., 1972: A simultaneous growth and yield model for loblolly pine. For. Sci. 18:76-86.

Trincado, G. und Gadow v. K., 1996: Zur Sortimentschätzung stehender Laubbäume. Centralblatt für das gesamte Forstwesen. Heft 1, S. 27-38. 



\section{Veröffentlichung II}

Analyse des Grundflächenzuwachses gleichaltriger Fichtenreinbestände

SÁnCHEZ Orois, S., GuRJanOv, M. und SCHRÖDER, J.

Erschienen in:

Allgemeine Forst- und Jagdzeitung. Jahrgang 2001, Heft 3:51-60. 



\section{Analyse des Grundflächenzuwachses gleichaltriger Fichtenreinbestände}

Analysis of basal area increment of even aged stands of Norway spruce

Herm Professor Dr. A. Akşa anlässlich seiner Pensionierung gewidmet

Sofía SÁNCHEZ-Orois, Michail GurJanOV u. JÖRg SCHRÖDER

Institut für Forsteinrichtung und Ertragskunde. Georg-August-Universität Göttingen

Büsgenweg 5, 37077 Göttingen. Germany

\section{Zusammenfassung}

Ziel der vorliegenden Arbeit ist die vergleichende Analyse verschiedener Grundflächenzuwachsmodelle, die dem Differentialgleichung-Typ (DIF) zuzuordnen sind. Die zur Parametrisierung der Modelle verwendeten Daten (Tab. 1), die von der Niedersächsischen Forstlichen Versuchsanstalt zur Verfügung gestellt wurden, stammen von 87 gleichaltrigen Fichtenreinbeständen (Picea abies L. Karst). Unter den analysierten Modellen (Gl. 4-6) zeigt die Funktion von Rodríguez (Gl. 6) die beste Anpassung (s. Tab. 3 u. 4; Abb. 2). Ein Vergleich zwischen den Modellen der pfad-invarianten algebraischen Differenzform (PID) und den untersuchten DIF-Modellen wurde durchgeführt. Weil die PID-Modelle die Grundfläche schätzen und die DIF-Modelle den laufenden Grundflächenzuwachs, sind beide Gleichungstypen zunächst nicht vergleichbar. Deshalb war es notwendig, die untersuchten Differentialgleichungen in den PID-Typ (Gl. 10-12) zu transformieren. Aus den Ergebnissen (s. Tab. 7 u. 8; Abb. 5) kann abgeleitet werden, dass die verwendeten PID-Modelle (Gl. 10-12) eine bessere Anpassung als die Modelle (Gl. 13-14) ergeben. Unter ihnen zeigt das Modell von Rodríguez (Gl. 12) die beste Anpassung.

Schlagwörter: Grundfläche; Grundflächenzuwachs; Grundflächenmodellierung; Fichtenbestände. 


\section{Einleitung}

Die forstbetriebliche Planung benötigt Simulationsmodelle zur Prognose des Waldwachstums und der Holzerträge von Beständen. Flexible Wuchsmodelle ermöglichen dem Wissenschaftler Einblicke in die komplizierten Wachstumsprozesse des Waldes. Sie bilden die Grundlage für ein tieferes Verständnis der Walddynamik und ermöglichen eine Analyse unterschiedlicher Eingriffsvarianten für reale Bestände (Wenk, 1990; Gadow, 1992; Pretzsch, 1992; Nagel, 1995). Für die forstliche Praxis sind Simulationsmodelle unverzichtbare Instrumente zur Unterstützung der Planung und Entscheidungsfindung.

Ein zentrales Element eines Wuchsmodells ist die Prognose der Bestandesgrundfläche; ein wichtiges Dichtemaß, das im Zusammenhang mit dem Durchmesser und der Stammzahl eines Bestandes steht. Zusammen mit der Stammzahl kann dieser Parameter außerdem dazu benutzt werden, Durchforstung-Programme zu definieren (z.B. Álvarez González, 1997; Staupendahl, 1999). Darüber hinaus ist die Kenntnis der Grundfläche auch wichtig, um das Verhältnis zwischen Bestockungsgrad und Volumenzuwachs zu untersuchen (Assmann, 1961).

Es gibt zwei Prozesse, die die Grundflächenentwicklung eines Bestandes beeinflussen: Das natürliche Wachstum und die forstlichen Eingriffe. Diese Prozesse sind schematisch in Abbildung 1 dargestellt.

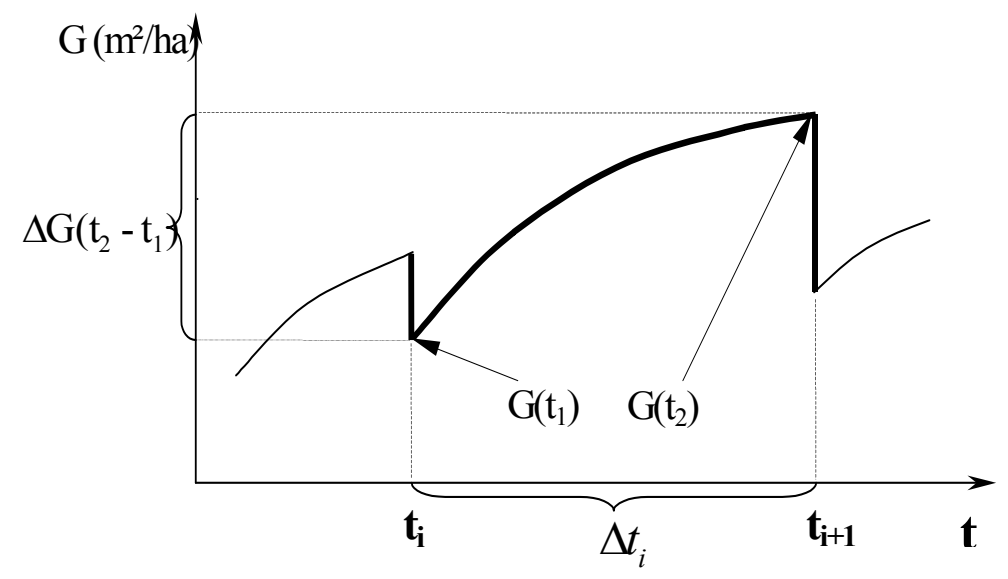

Abb. 1. Schematische Abfolge von Wachstum und Eingriff ( $G=$ Grundfläche $\left[\mathrm{m}^{2} / \mathrm{ha}\right], G\left(t_{1}\right)$ und $G\left(t_{2}\right)=$ Grundfläche im Alter $t_{1}$ und $t_{2},\left[m^{2} / h a\right], \Delta G\left(t_{1}-t_{2}\right)=$ Zunahme der Grundfläche durch Wachstum $\left[\mathrm{m}^{2} / \mathrm{ha}\right], \Delta t=$ Messintervall zwischen forstlichen Eingriffen [Jahre]).

Fig. 1. Schematic sequence of growth and removals $\left(G=\right.$ Basal area $\left[\mathrm{m}^{2} / \mathrm{ha}\right], G\left(t_{1}\right)$ and $G\left(t_{2}\right)=$ Basal area at age $t_{1}$ and $t_{2},\left[\mathrm{~m}^{2} / \mathrm{ha}\right], \Delta G\left(t_{1}-t_{2}\right)=$ Basal area increment $\left[\mathrm{m}^{2} / \mathrm{ha}\right], \Delta t=$ Time interval between removals [years]). 
Bestandesgrundflächenmodelle lassen sich in zwei Gruppen unterteilen:

1. Pfad-invariante algebraische Differenzform Modelle (PID-Typ), die die Grundfläche im Alter $\left(A_{2}\right)$ als Funktion von Grundfläche, Höhe und Stammzahl im Alter $\left(A_{1}\right)$ und Höhe und Stammzahl im Alter $\left(\mathrm{A}_{2}\right)$ bestimmen (z.B. Schumacher, 1939; Souter, 1986; Gadow u. Hui, 1993, 1999; Forss, 1994).

2. Differentialgleichungen zur Modellierung des laufenden Grundflächenzuwachses (DIFTyp). Beispiele sind die auf dem Zustandsraum-Verfahren (state space approach) basierenden Modelle von García (1994), Rodríguez Soalleiro (1995) und Kvist Johannsen (1999).

$\mathrm{Zu}$ den früheren deutschen Beiträgen im Zusammenhang mit der Anwendung von DIFModellen und ihren Approximationen zählen die Arbeiten von Sloboda (1971) und Hradetzky (1972).

Ziel der vorliegenden Arbeit ist es, die Eignung verschiedener DIF Modelle für durchforstete, gleichaltrige Fichtenbestände zu prüfen und eine vergleichende Analyse zwischen beiden Modelltypen durchzuführen. Im Einzelnen sollen dabei die Ansätze von García (1994), Rodríguez Soalleiro (1995) sowie Kvist Johannsen (1999) untersucht werden.

\section{Daten}

Zur Parametrisierung der Modelle wurden die Daten von 87 Fichtenversuchsflächen verwendet ${ }^{1}$. Insgesamt stehen 649 Beobachtungswerte mit Alter, Höhe, Stammzahl, Grundfläche am Anfang und am Ende der Zeitperiode zwischen zwei forstlichen Eingriffen zur Verfügung. Die durchschnittlichen Werte einiger Bestandesparameter sowie eine Sortierung der Daten nach Dauer des Messintervalls zwischen den Messungen findet sich in Tabelle 1.

\footnotetext{
${ }^{1}$ Die von Schübeler (1997) aufbereiteten Daten wurden freundlicherweise von der Niedersächsischen Forstlichen Versuchsanstalt zur Verfügung gestellt.
} 


\begin{tabular}{|c|c|c|c|c|c|c|}
\hline $\begin{array}{c}\text { Messintervall } \\
\text { (Jahre) }\end{array}$ & $\begin{array}{c}\text { Anzahl der } \\
\text { Messungen }\end{array}$ & $\mathrm{A}_{\mathrm{m}}$ & $\mathrm{H}_{\mathrm{m}}$ & $\mathrm{G}_{\mathrm{m}}$ & $\frac{d G}{d t}$ & $\frac{d H}{d t}$ \\
\hline $0.5-1.5$ & 16 & 88,813 & 25,781 & 43,587 & 0,948 & 0,244 \\
\hline $1.5-2.5$ & 38 & 77,845 & 24,603 & 39,194 & 0,923 & 0,242 \\
\hline $2.5-3.5$ & 78 & 79,653 & 25,771 & 42,823 & 0,904 & 0,210 \\
\hline $3.5-4.5$ & 174 & 74,525 & 25,231 & 40,593 & 0,872 & 0,227 \\
\hline $4.5-5.5$ & 167 & 79,711 & 23,792 & 41,490 & 0,775 & 0,183 \\
\hline $5.5-6.5$ & 50 & 80,360 & 24,556 & 40,880 & 0,770 & 0,168 \\
\hline $6.5-7.5$ & 57 & 64,428 & 21,291 & 37,178 & 0,868 & 0,211 \\
\hline $7.5-8.5$ & 27 & 62,156 & 20,292 & 37,734 & 0,937 & 0,247 \\
\hline $8.5-9.5$ & 9 & 80,011 & 25,444 & 34,377 & 0,599 & 0,161 \\
\hline $9.5-10.5$ & 14 & 74,250 & 21,793 & 40,115 & 0,719 & 0,161 \\
\hline $10.5-11.5$ & 12 & 59,800 & 19,700 & 35,143 & 0,862 & 0,195 \\
\hline $11.5-15.5$ & 7 & 76,143 & 21,428 & 40,843 & 0,606 & 0,155 \\
\hline $\begin{array}{c}\text { Summe/ } \\
\text { Mittelwert }\end{array}$ & 649 & 75,9 & 24,08 & 40,49 & 0,840 & 0,206 \\
\hline
\end{tabular}

Tab. 1. Durchschnittliche Werte einiger Bestandesparameter für die Gesamtdaten und sortiert nach der Dauer des Messintervalls (Am = durchschnittliches Alter [Jahre], Hm = durchschnittliche Höhe [m], $G m=$ durchschnittliche Grundfläche $\left[\mathrm{m}^{2} / \mathrm{ha}\right], d G / d t=$ durchschnittlicher Grundflächenquwachs $\left[\mathrm{m}^{2} / \mathrm{ha} \cdot J a h r\right], d H / d t=$ durchschnittlicher Höhenzuwachs $[\mathrm{m} / \mathrm{ha} \cdot J a h r]$. Daten aus Schübeler, 1997).

Tab. 1. Mean values of some parameters of the stand for the whole data set and according to the length of the time interval $\left(\mathrm{Am}=\right.$ mean age [years], $\mathrm{Hm}=$ mean height $[\mathrm{m}], \mathrm{Gm}=$ mean basal area $\left[\mathrm{m}^{2} / \mathrm{ha}\right]$, $d G / d t=$ mean basal area annual increment $\left[\mathrm{m}^{2} / \mathrm{ha} \cdot J \mathrm{Jahr}\right], d H / d t=$ mean annual height increment $[\mathrm{m} / \mathrm{ha} \cdot J a h r]$. Data from Schübeler, 1997).

Einige Rohdatensätze, die zur Anpassung der Modelle verwendet wurden, werden in der Tabelle 2 aufgelistet:

\begin{tabular}{|c|c|c|c|c|c|c|}
\hline $\mathrm{t}_{\mathrm{i}}$ & $\mathrm{N}_{\mathrm{i}}$ & $\mathrm{G}_{\mathrm{i}}$ & $\mathrm{G}_{\mathrm{i}+1}$ & $\mathrm{H}_{\mathrm{i}}$ & $\mathrm{H}_{\mathrm{i}+1}$ & $\frac{d G_{i}}{d t_{i}}$ \\
\hline 36,0 & 1524 & 33,25 & 45,2 & 15,3 & 18,31 & 1,49 \\
\hline 44,0 & 1296 & 42,44 & 48,06 & 19,0 & 20,03 & 1,87 \\
\hline 47,0 & 1088 & 44,94 & 50,16 & 20,8 & 22,09 & 1,09 \\
\hline 51,8 & 852 & 44,89 & 46,86 & 23,0 & 23,43 & 0,78 \\
\hline 54,3 & 728 & 43,84 & 48,41 & 24,1 & 25,11 & 1,24 \\
\hline 58,0 & 684 & 47,20 & 50,35 & 25,4 & 26,24 & 0,90 \\
\hline 61,5 & 632 & 48,70 & 53,69 & 26,6 & 27,78 & 1,16 \\
\hline 65,8 & 596 & 52,56 & 54,59 & 28,1 & 28,73 & 0,68 \\
\hline 68,8 & 540 & 50,87 & 54,37 & 28,9 & 29,66 & 0,83 \\
\hline 73,0 & 460 & 48,88 & 51,19 & 30,0 & 30,45 & 0,77 \\
\hline$\ldots$. & $\ldots$. & $\ldots$. & $\ldots$. & $\ldots$. & $\ldots$. & $\ldots$ \\
\hline
\end{tabular}

Tab. 2. Ein Beispiel der für die Anpassung der Modelle verwendeten Datensätze ( $t_{i}=$ Alter [Jabre]; $N_{i}=$ Stammzahl pro ha.; $G_{i}$ und $G_{i+1}=$ Grundfläche $\left[\mathrm{m}^{2} / \mathrm{ha}\right] ; H_{i}$ und $H_{i+1}=H \ddot{b}$ e $[\mathrm{m}] ; d G_{i} / d t_{i}=$ Grundflächenzuwachsrate $\left.\left[\mathrm{m}^{2} / \mathrm{ha} \cdot \mathrm{Jahr}\right]\right)$.

Tab. 2. An example of the data used to fit the models $\left(t_{i}=\right.$ age [years $] ; N_{i}=$ stem number pro ha.; $G_{i}$ und $G_{i+1}=$ basal area $\left[\mathrm{m}^{2} / \mathrm{ha}\right] ; H_{i}$ und $H_{i+1}=$ height $[\mathrm{m}] ; d G_{i} / d t_{i}=$ basal area increment $\left.\left[m^{2} / h a \cdot J a h r\right]\right)$. 


\section{Dif-Modelle}

Das Hauptmerkmal, das die beschriebenen DIF-Typ Modelle charakterisiert, ist, dass die abhängige Variable eine Wachstumsrate ist, die dynamisch geschätzt wird. In dieser Arbeit wurden die Modelle von García (1994), Rodríguez (1995) und Kvist Johannsen (1999) analysiert.

Das von García (1994) vorgelegte zweidimensionale state space Modell beruht auf dem laufenden Grundflächenzuwachs- und Höhenzuwachsmodell von Clutter (1963) und Clutter und Lenhart (1971).

$$
\begin{aligned}
& \frac{d H}{d t}=a \cdot H \cdot(b-\ln H)^{2} \\
& \frac{d G}{d t}=a \cdot G \cdot(c-\ln G) \cdot(b-\ln H)
\end{aligned}
$$

Um dieses autonome System anzupassen, wurde als erster Schritt das Integral der Gleichung 1 berechnet. Die Lösung der Gleichung 1 lautet:

$$
H=e^{b-\frac{1}{a t+d}}
$$

Nach dem Ersetzen der Lösung in der Gleichung 2 lautet die Differentialgleichung zur Schätzung des Grundflächenzuwachses wie folgt:

$$
\frac{d G}{d t}=a \cdot G \cdot(c-\ln G) \cdot \frac{1}{a \cdot t+d}
$$

Gleichung 4 wurde für die Anpassung verwendet. Die ermittelten Parameter werden in der Tabelle 3 aufgelistet.

Das nachstehende Modell (s. Gl. 5) wurde von Kvist Johannsen (1999) in Dänemark entwickelt. Die Modellparameter haben bei der für 52 einzelne Eichenbestände durchgeführten Überprüfung eine gute Anpassung gezeigt. 


$$
\frac{d G}{d t}=a \cdot G^{b} \cdot e^{(-c \cdot G-d \cdot H)}
$$

Die von Rodríguez (1995) vorgeschlagene Funktion ist Teil eines Bestandessimulators zur Prognose gleichaltriger Reinbestände der Baumart Pinus pinaster. Sie wurde auf Basis der von Alder (1980) vorgelegten dynamischen Modelle zur Prognose des Grundflächenzuwachses entwickelt (s. Gl. 6).

$$
\begin{array}{ll}
\frac{d G}{d t}=a \cdot G^{b} \cdot t^{c} & \\
\text { wobei } \mathrm{dG} / \mathrm{dt} & =\text { laufender Grundflächenzuwachs }\left(\mathrm{m}^{2} / \mathrm{ha} \cdot J a h r\right) \\
\mathrm{G} & =\text { Grundfläche }\left(\mathrm{m}^{2} / \mathrm{ha}\right) ; \\
\mathrm{t} & =\text { Alter (Jahre); } \\
\mathrm{a}, \mathrm{b} \text { und c } & =\text { Modellparameter. }
\end{array}
$$

Alle genannten Modelle wurden bereits zur Beschreibung der Bestandesgrundflächenentwicklung bei verschiedenen Baumarten eingesetzt. Die Berechnungen wurden mit Hilfe des statistischen Programmpakets STATISTICA nach der nichtlinearen Methode der kleinsten Quadrate durchgeführt.

Die Gleichungen (4)-(6) sind den dynamischen Modellen zuzuordnen. Die wichtigsten Vorteile, die der dynamische Ansatz bietet, sind (Alder, 1980):

- Diese Modelle erklären besser als die statischen Modelle die Beziehungen zwischen der Bestandesdichte und dem Ertrag, vor allem, weil in dynamischen Modellen das Wachstum direkt durch die Durchforstungsstärke beeinflusst wird, ebenso wie die Bestandesdichte auf die folgende Durchforstung wirkt. Die dynamischen Modelle sind hilfreich, um ein breites Spektrum waldbaulicher Behandlungsprogramme zu simulieren.

- Zur Parametrisierung der Modelle können Daten verwendet werden, die aus sehr unterschiedlichen Versuchsflächen stammen. Dabei kann die bestandesgeschichtliche Entwicklung der einzelnen Bestände vernachlässigt werden. 


\section{Ergebnisse und Diskussion}

Die Parametrisierung der Gleichungen erfordert die Information über Änderungsraten, die sich auf ein infinitesimales Zeitintervall beziehen. Wegen des Mangels z.B. an jährlichen Daten wurden für die Schätzung der Modellparameter die Wachstumsraten für drei-, vierund fünfjährige Zeitintervalle approximiert und alternativ verwendet. Die Zielfunktionen, die zur Berechnung der Parameter der Gleichungen (4)-(6) benutzt wurden, lauten wie folgt:

$$
\begin{aligned}
& \sum\left(\left[\frac{d G}{d t}\right]_{i}-a \cdot G_{i} \cdot\left(c-\ln G_{i}\right) \cdot \frac{1}{a \cdot t_{i}+d}\right)^{2}=\operatorname{Min} \\
& \sum\left(\left[\frac{d G}{d t}\right]_{i}-a \cdot G_{i}^{b} \cdot e^{\left(-c \cdot G_{i}-d \cdot H_{i}\right)}\right)^{2}=\operatorname{Min} \\
& \sum\left(\left[\frac{d G}{d t}\right]_{i}-a \cdot G_{i}{ }^{b} \cdot t_{i}^{c}\right)^{2}=\operatorname{Min}
\end{aligned}
$$

wobei $\mathrm{dG} / \mathrm{dt} \quad=$ laufender Grundflächenzuwachs ( $\left.\mathrm{m}^{2} / \mathrm{ha} \cdot J a h r\right)$;

G $\quad=$ Grundfläche $\left(\mathrm{m}^{2} / \mathrm{ha}\right)$;

$\mathrm{t} \quad=$ Alter (Jahre);

$\mathrm{a}, \mathrm{b}, \mathrm{c}$ und $\mathrm{d}=$ Modellparameter.

In der Tabelle 3 werden die für die untersuchten Modelle geschätzten Modellparameter nach Dauer des Messintervalls aufgelistet. Um die Anpassungsgüte der berücksichtigten Modelle bewerten und vergleichen zu können, wurde der Korrelationskoeffizient (Ryŷ) zwischen den gemessenen und aus dem Modell berechneten Wachstumsraten verwendet. Zusätzlich wurden zur Evaluierung der Modelle die Wurzel des mittleren quadratischen Fehlers (RMSE) sowie das mittlere Residuum (MRES) verwendet. 


\begin{tabular}{|c|c|c|c|c|c|c|c|}
\hline Messintervall & \multicolumn{4}{|c|}{ Geschätzte Modellparameter } & & \multicolumn{2}{|c|}{ Absolut } \\
\hline & $\mathrm{a}$ & $\mathrm{b}$ & $\mathrm{c}$ & $\mathrm{d}$ & Ryŷ & MRES & RMSE \\
\hline García & 0,332 & & 5,102 & 2,533 & 0,565 & 0,0237 & 0,0426 \\
\hline Kvist Johannsen & 11,018 & $-0,696$ & $-0,024$ & 0,037 & 0,536 & $-0,0016$ & 0,0436 \\
\hline Rodríguez & 22,805 & 0,103 & $-0,843$ & & 0,593 & 0,0010 & 0,0416 \\
\hline $\begin{array}{l}\text { Messintervall } \\
\Delta \mathrm{t}=4 \text { Jahre }\end{array}$ & \multicolumn{4}{|c|}{ Geschätzte Modellparameter } & & \multicolumn{2}{|c|}{ Absolut } \\
\hline & $\mathrm{a}$ & $\mathrm{b}$ & c & $\mathrm{d}$ & Ryŷ & MRES & RMSE \\
\hline García & 0,046 & & 5,287 & 0,349 & 0,717 & 0,0228 & 0,0167 \\
\hline Kvist Johannsen & 0,580 & 0,496 & 0,008 & 0,044 & 0,716 & 0,0001 & 0,0167 \\
\hline Rodríguez & 18,491 & $-0,008$ & $-0,720$ & & 0,765 & 0,0000 & 0,0155 \\
\hline $\begin{array}{c}\text { Messintervall } \\
\Delta t=5 \text { Jahre }\end{array}$ & \multicolumn{4}{|c|}{ Geschätzte Modellparameter } & & \multicolumn{2}{|c|}{ Absolut } \\
\hline & $\mathrm{a}$ & $\mathrm{b}$ & $\mathrm{c}$ & $\mathrm{d}$ & Ryŷ & MRES & RMSE \\
\hline García & 0,145 & & 5,074 & 0,178 & 0,792 & 0,0103 & 0,0138 \\
\hline Kvist Johannsen & 66,032 & $-1,455$ & $-0,044$ & 0,038 & 0,564 & 0,0025 & 0,0187 \\
\hline Rodríguez & 21,241 & 0,154 & $-0,904$ & & 0,804 & 0,0007 & 0,0135 \\
\hline
\end{tabular}

Tab. 3. Parameterwerte der Grundflächenmodelle sowie Absolut- und Relativwerte der Kriterien der Anpassungsgenanigkeit für drei-, vier-, und fünfjährige Messintervalle; Ryy $=$ Korrelationskoeffizient; MRES = Mittelwert der Residuen; RMSE $=$ Wurzel des mittleren quadratischen Feblers.

Tab. 3. Values of the parameters as well as absolute and relative values of the criteria for analysing the model accuracy: $R_{y \hat{y}}=$ coefficient of correlation; $M R E S=$ mean residual; $R M S E=$ root mean square error.

Wie aus Tabelle 3 zu ersehen ist, zeigt das Modell von Rodríguez über alle drei $\Delta \mathrm{t}$-Varianten die beste Anpassungsgenauigkeit. Ein Grund dafür ist die Altersabhängigkeit, da es eine deutliche Beziehung zwischen Alter und Grundflächenzuwachs gibt.

In Tabelle 4 sind die berechneten MRES-Werte und RMSE-Werte nach Altersklassen aufgelistet. Abbildung 2 veranschaulicht diese Ergebnisse graphisch.

\begin{tabular}{|c|c|c|c|c|c|c|}
\hline & \multicolumn{3}{|c|}{ MRES } & \multicolumn{3}{c|}{ RMSE } \\
\hline Altersbereich & Rodríguez & García & Johannsen & Rodríguez & García & Johannsen \\
\hline $20-30$ & $-0,0015$ & $-0,0006$ & 0,0009 & 0,0115 & 0,0131 & 0,0144 \\
\hline $30-40$ & 0,0041 & 0,0030 & 0,0097 & 0,0245 & 0,0403 & 0,0466 \\
\hline $40-50$ & $-0,0022$ & $-0,0063$ & $-0,0024$ & 0,0480 & 0,0712 & 0,0401 \\
\hline $50-60$ & $-0,0031$ & $-0,0013$ & 0,0001 & 0,0805 & 0,0806 & 0,0981 \\
\hline $60-70$ & $-0,0074$ & $-0,0083$ & 0,0116 & 0,0973 & 0,0920 & 0,1147 \\
\hline $70-80$ & 0,0066 & 0,0110 & 0,0043 & 0,1223 & 0,1066 & 0,1173 \\
\hline $80-90$ & 0,0058 & 0,0075 & $-0,0041$ & 0,0953 & 0,0718 & 0,0784 \\
\hline $90-100$ & $-0,0028$ & 0,0039 & $-0,0027$ & 0,0925 & 0,0577 & 0,0860 \\
\hline $100-110$ & 0,0060 & 0,0082 & $-0,0045$ & 0,0780 & 0,0552 & 0,0592 \\
\hline $110-120$ & $-0,0040$ & 0,0016 & $-0,0098$ & 0,0575 & 0,0318 & 0,0745 \\
\hline $120-130$ & 0,0011 & 0,0036 & 0,0013 & 0,0599 & 0,0506 & 0,0514 \\
\hline
\end{tabular}

Tab. 4. Nach Altersklassen stratifizierte MRES- und RMSE Werte.

Tab. 4. MRES- and RMSE values according to age-classes. 


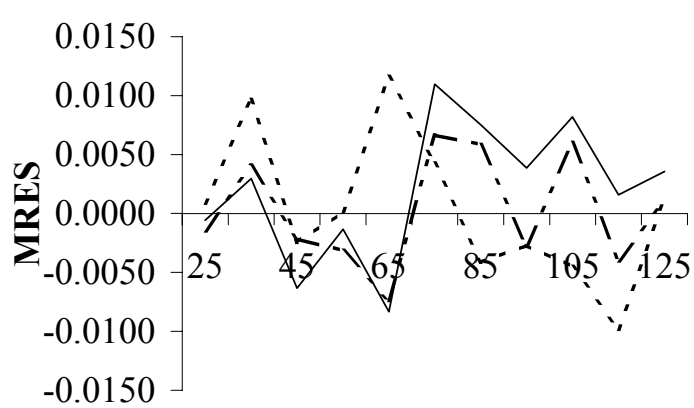

Altersklasse

(a)

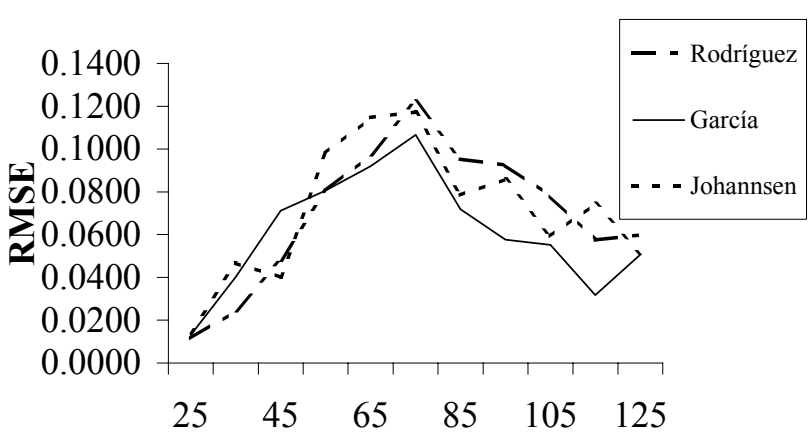

Altersklasse

(b)

Abb. 2. Nach Altersklassen stratifizierte Werte für MRES (a) und RMSE (b).

Fig. 2. MRES (a) and RMSE (b) according to age-classes.

Ein weiteres Ziel dieser Arbeit ist die vergleichende Analyse zwischen den Modellen des PIDund DIF-Typs. Die Modelle des ersten Typs haben bei früheren Untersuchungen eine höhere Anpassungsgenauigkeit gezeigt. So variieren die Werte des Korrelationskoeffizienten (Ryŷ) von 0,989 bis 0,994 (Gurjanov et al., 2000). Um die PID- mit den DIF-Modellen vergleichen zu können, ist es nötig, die Differentialgleichungen von Rodríguez, García und Johannsen in die pfad-invariante algebraische Differenzenform zu transformieren.

Die in die PID-Form umgewandelte Gleichung von García lautet dann wie folgt:

$$
G_{2}=G_{1}+\left[a \cdot G_{1} \cdot\left(c-\ln G_{1}\right) \cdot \frac{1}{a \cdot t+d}\right] \cdot \Delta t
$$

wobei $\mathrm{G}_{1}$ und $\mathrm{G}_{2}=$ Grundfläche im Alter $\mathrm{t}_{1}$ und $\mathrm{t}_{2}\left(\mathrm{~m}^{2} / \mathrm{ha} \cdot \mathrm{Jahr}\right)$;

$\mathrm{H}_{1} \quad=$ Oberhöhe im Alter $\mathrm{t}_{1}(\mathrm{~m})$;

$\Delta \mathrm{t} \quad=$ Zeitintervall (Jahre);

$\mathrm{a}$, und $\mathrm{c} \quad=$ Modellparameter.

Die folgende Gleichung entspricht der Umwandlung der Differentialgleichung von Kvist Johannsen in eine pfad-invariante algebraische Differenzenform:

$$
G_{2}=G_{1}+\left(a \cdot G_{1}^{b} \cdot e^{\left(-c \cdot G_{1}-d \cdot H_{1}\right)}\right) \cdot \Delta t
$$

Die Formulierung der Differentialgleichung von Rodríguez nach der Umwandlung in den PID-Typ lautet:

$$
G_{2}=G_{1}+\left(a \cdot G_{1}^{b} \cdot t_{1}^{c}\right) \cdot \Delta t
$$

Die in PID-Modelle umgewandelten Gleichungen (10-12) stellen die ersten Annäherungen des Integrals der Differentialgleichungen für das Intervall $\left(\mathrm{t}_{1}, \mathrm{t}_{2}\right)$ dar. 
Auch für diese Gleichungen wurden die Modellparameter jeweils für unterschiedliche Messintervalle mit der nichtlinearen Methode der kleinsten Quadrate geschätzt. Um die Anpassungsgenauigkeit vergleichen zu können, wurden die bereits oben genannten Kriterien verwendet. Tabelle 5 zeigt die ermittelten Parameter und Kriterien.

\begin{tabular}{|c|c|c|c|c|c|c|c|}
\hline Messintervall & \multicolumn{4}{|c|}{ Geschätzte Modellparameter } & & \multicolumn{2}{|c|}{ Absolut } \\
\hline & $\mathrm{a}$ & $\mathrm{b}$ & $\mathrm{c}$ & $\mathrm{d}$ & Ryŷ & MRES & RMSE \\
\hline García (Gl.10) & 0,262 & & 5,124 & $-1,982$ & 0,992 & 0,0487 & 0,1268 \\
\hline Kvist Johannsen (Gl.11) & 11,177 & $-0,705$ & $-0,024$ & 0,037 & 0,992 & $-0,0143$ & 0,1312 \\
\hline Rodríguez (Gl.12) & 23,022 & 0,133 & $-0,869$ & & 0,993 & $-0,0091$ & 0,1243 \\
\hline $\begin{array}{c}\text { Messintervall } \\
\Delta \mathrm{t}=4 \mathrm{Jahre}\end{array}$ & \multicolumn{4}{|c|}{ Geschätzte Modellparameter } & & \multicolumn{2}{|c|}{ Absolut } \\
\hline & $\mathrm{a}$ & $\mathrm{b}$ & c & $\mathrm{d}$ & Ryŷ & MRES & RMSE \\
\hline García (Gl.10) & 0,781 & & 5,286 & 5,828 & 0,995 & 0,0873 & 0,0655 \\
\hline Kvist Johannsen (Gl.11) & 0,642 & 0,465 & 0,007 & 0,045 & 0,995 & $-0,0027$ & 0,0651 \\
\hline Rodríguez (Gl.12) & 18,012 & 0,006 & $-0,725$ & & 0,996 & 0,0000 & 0,0608 \\
\hline $\begin{array}{c}\text { Messintervall } \\
\Delta \mathrm{t}=5 \mathrm{Jahre}\end{array}$ & \multicolumn{4}{|c|}{ Geschätzte Modellparameter } & & \multicolumn{2}{|c|}{ Absolut } \\
\hline & $\mathrm{a}$ & $\mathrm{b}$ & $\mathrm{c}$ & $\mathrm{d}$ & Ryŷ & MRES & RMSE \\
\hline García (Gl.10) & 0,028 & & 5,078 & $-0,033$ & 0,996 & 0,0473 & 0,0685 \\
\hline Kvist Johannsen (Gl.11) & 74,037 & $-1,503$ & $-0,045$ & 0,037 & 0,992 & 0,0184 & 0,0922 \\
\hline Rodríguez (Gl.12) & 21,093 & 0,156 & $-0,903$ & & 0,996 & 0,0022 & 0,0669 \\
\hline
\end{tabular}

Tab. 5. Parameterwerte der Grundflächenmodelle sowie Absolut- und Relativwerte der Kriterien der Anpassungsgenanigkeit für drei-, vier-, und fünfjäbrige Messintervalle: Ryy = Korrelationskoeffizient; MRES = Mittelwert der Residuen; RMSE = Wurzel des mittleren quadratischen Feblers.

Tab. 5. Values of the parameters as well as absolute and relative values of the criteria for analysing the model accuracy: $\mathrm{R}_{y \hat{y}}=$ coefficient of correlation; $M R E S=$ mean residual; $\mathrm{RMSE}=$ root mean square error.

Wie aus Tabelle $5 \mathrm{zu}$ entnehmen ist, gibt es zwischen den Modellen lediglich geringe Unterschiede in der Genauigkeit der Anpassung. Die Werte des Korrelationskoeffizienten (Ryŷ) variieren zwischen 0,991 und 0,996.

Ein Vergleich der Tabellen 3 und 5 zeigt deutlich, dass die Parameterwerte der Gleichungen 10, 11 und 12 den für die Differentialgleichungen ermittelten Werten sehr ähnlich sind. Dieses Ergebnis war zu erwarten, da die durchgeführte Umwandlung der Gleichungen in die PID-Form eine Art Approximation der Integrale der zugehörigen Differentialgleichungen liefert. Ein Grund für die Unterschiede liegt darin, dass es in unserem Datensatz geringe Unterschiede hinsichtlich der Dauer des Messintervalls gibt. Wenn ausschließlich Daten verwendet werden, die sich auf ein konstantes Messintervall beziehen, sind die Werte der geschätzten Modellparemeter beinahe identisch. 
Trotz der Ähnlichkeit der Werte der Modellparameter, gibt es einen offensichtlichen Unterschied im Korrelationskoeffizienten (Ryŷ) zwischen den Differential- und den PIDGleichungen. Ein Grund dafür ist, dass es zwischen der Grundfläche im Alter $t_{1}$ und $t_{2}$ eine höhere Korrelation als zwischen dem laufenden Grundflächenzuwachs und der Grundfläche gibt, dies ist in der Abbildung 3 zu ersehen.

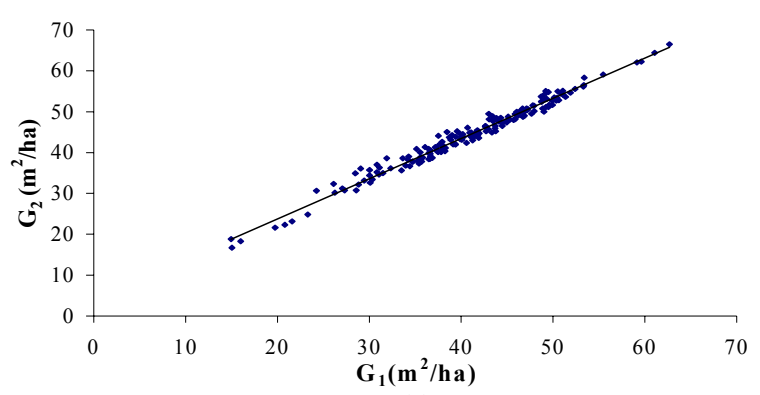

(a)

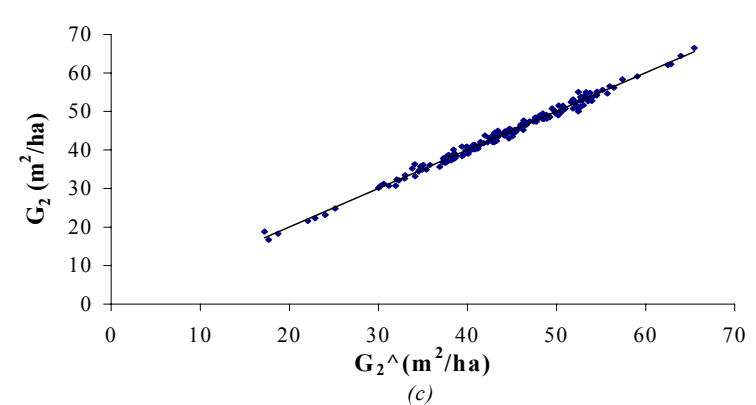

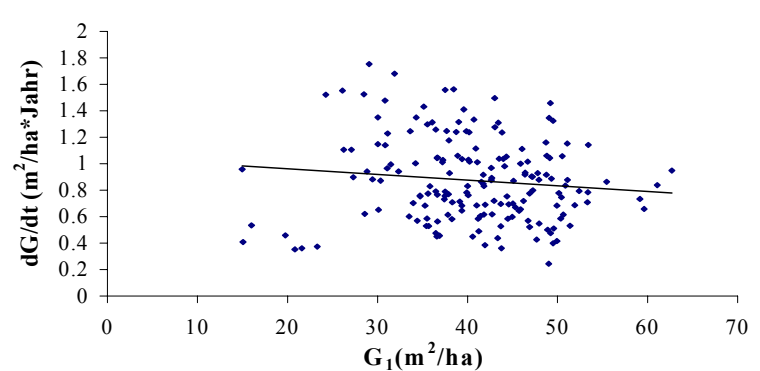

(b)

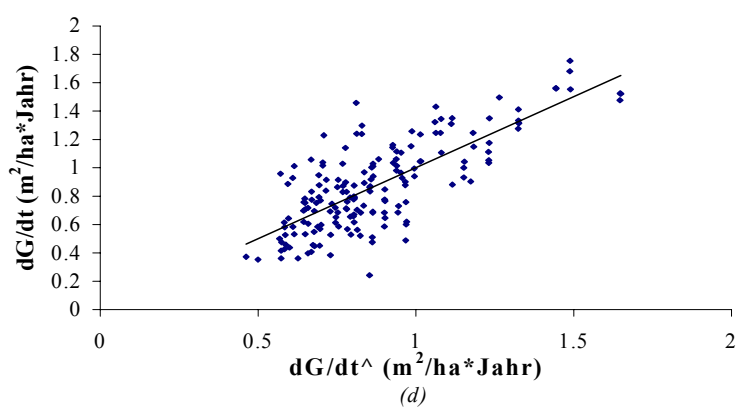

Abb. 3. Beziehung zwischen den beobachteten Werten der Grundfläche im Alter t2 und t1 (a); Beziehung zwischen den beobachteten Werten des Grundflächenzwwachses und der Grundfläche im Alter 11 (b); beobachtete über den nach dem verarbeiteten Rodríguez. Modell theoretischen Grundflächenwerten (c); beobachtete über den nach dem Rodriguez. Modell theoretischen Grundflächenzuwachswerten.

Fig. 3. Relation between the observed values of the basal area at age $t_{2}$ and $t_{1}$ (a); Relation between the observed values of the annual increment and the basal area at age $t_{1}(b)$; observed over predicted values of the basal area calculated according to Rodriguez modified model (c); observed over predicted values of the annual increment calculated according to Rodriguez model.

Wie in den Abbildungen 3 (a) und (b) zu ersehen ist, nimmt der Korrelationskoeffizient zwischen zwei Variablen (z.B. $G_{2}$ und $G_{1}$ ) immer höhere Werte an als der Korrelationskoeffizient zwischen einer der beiden Variablen und der Differenz der Variablen (in unserem Fall $G_{1}$ und $d G$ als die Differenz $G_{2}-G_{1}$; s. z.B. die Arbeit von Lappi, 1993).

In Tabelle 6 sind für die verarbeiteten Gleichungen die berechneten MRES-Werte und RMSE-Werte nach Altersklassen aufgelistet. Abbildung 4 veranschaulicht diese Ergebnisse. 


\begin{tabular}{|c|c|c|c|c|c|c|}
\hline & \multicolumn{3}{|c|}{ MRES } & \multicolumn{3}{c|}{ RMSE } \\
\hline Altersbereich & $\begin{array}{c}\text { García } \\
(\text { Gl.10) }\end{array}$ & $\begin{array}{c}\text { Johannsen } \\
(\text { Gl.11) }\end{array}$ & $\begin{array}{c}\text { Rodríguez } \\
(\text { Gl.12) }\end{array}$ & $\begin{array}{c}\text { García } \\
(\text { Gl.10) }\end{array}$ & $\begin{array}{c}\text { Johannsen } \\
(\text { Gl.11 })\end{array}$ & $\begin{array}{c}\text { Rodríguez } \\
(\text { Gl.12) }\end{array}$ \\
\hline $20-30$ & $-0,0001$ & 0,0001 & $-0,0003$ & 0,0016 & 0,0025 & 0,0023 \\
\hline $30-40$ & 0,0004 & 0,0012 & 0,0006 & 0,0044 & 0,0062 & 0,0035 \\
\hline $40-50$ & $-0,0004$ & $-0,0003$ & $-0,0002$ & 0,0062 & 0,0046 & 0,0049 \\
\hline $50-60$ & 0,0000 & $-0,0003$ & $-0,0002$ & 0,0074 & 0,0097 & 0,0072 \\
\hline $60-70$ & $-0,0005$ & 0,0009 & $-0,0004$ & 0,0075 & 0,0076 & 0,0069 \\
\hline $70-80$ & 0,0011 & 0,0002 & 0,0005 & 0,0098 & 0,0085 & 0,0088 \\
\hline $80-90$ & 0,0007 & $-0,0004$ & 0,0003 & 0,0076 & 0,0058 & 0,0070 \\
\hline $90-100$ & 0,0003 & $-0,0005$ & $-0,0004$ & 0,0050 & 0,0068 & 0,0066 \\
\hline $100-110$ & 0,0009 & $-0,0004$ & 0,0002 & 0,0056 & 0,0040 & 0,0045 \\
\hline $110-120$ & 0,0002 & $-0,0006$ & $-0,0003$ & 0,0029 & 0,0045 & 0,0032 \\
\hline $120-130$ & 0,0009 & 0,0003 & 0,0003 & 0,0125 & 0,0067 & 0,0076 \\
\hline
\end{tabular}

Tab. 6. Nach Altersklassen stratifizierte Werte für MRES (a) und RMSE (b).

Tab. 6. MRES (a) and RMSE (b) according to age-classes.

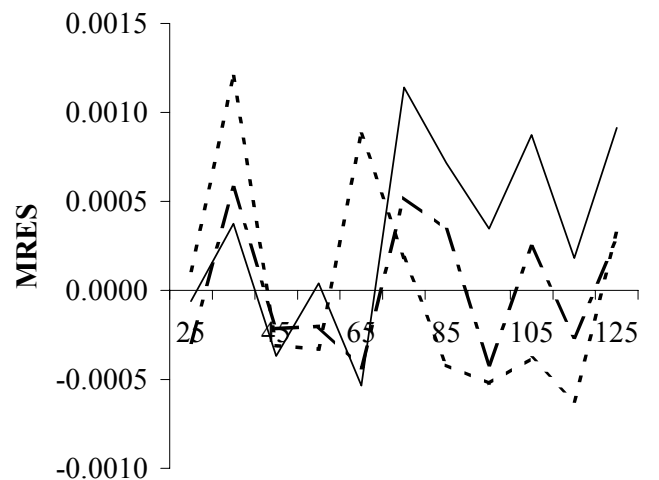

Altersklasse

(a)

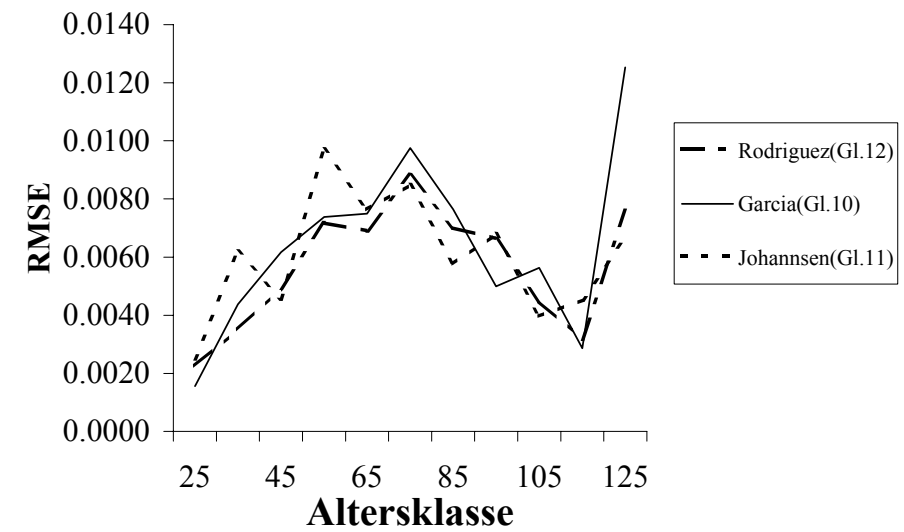

(b)

Abb. 4. Nach Altersklassen stratifizierte Werte für MRES (a) und RMSE (b).

Fig. 4. MRES (a) and RMSE (b) according to age-classes.

Die nachstehenden, zum PID-Typ gehörigen Grundflächenmodelle

$G_{2}=G_{1} \cdot N_{2}^{1-\alpha \cdot H_{2}^{\beta}} \cdot N_{1}^{\alpha \cdot H_{1}^{\beta}-1} \cdot\left(\frac{H_{2}}{H_{1}}\right)^{\delta}$ Hui und Gadow (1993)

$G_{2}=G_{1} \cdot\left(\frac{H_{2}}{H_{1}}\right)^{\delta}$ Hui (1997) 
wurden mit den PID-Gleichungstypen (10)-(12) von Rodríguez, García und Kvist Johannsen verglichen. Die Ergebnisse sind in Tabelle 6 aufgeführt. Im Gegensatz zu den DIF-Modellen (1)-(4) liefern die PID-Gleichungen (13) und (14) im Ergebnis qualitativ die gleiche Art der G-Prognose wie die PID-Gleichungen (10)-(12). Da die Zielgröße $\left(\mathrm{G}_{2}\right)$ gleich ist, ist auch ein echter quantitativer Vergleich der Maßzahlen der Anpassungsgüte wie Ryŷ und MRSE sowie RSME zwischen diesen Modellen möglich und auch sinnvoll.

\begin{tabular}{|c|c|c|c|c|c|c|c|}
\hline Messintervall & \multicolumn{4}{|c|}{ Geschätzte Modellparameter } & & \multicolumn{2}{|c|}{ Absolut } \\
\hline & $\mathrm{a}$ & $\mathrm{b}$ & $\mathrm{c}$ & $\mathrm{d}$ & Ryŷ & MRES & RMSE \\
\hline García (Gl.10) & 0,262 & & 5,124 & $-1,982$ & 0,992 & 0,0487 & 0,1268 \\
\hline Kvist Johannsen (Gl.11) & 11,177 & $-0,705$ & $-0,024$ & 0,037 & 0,992 & $-0,0143$ & 0,1312 \\
\hline Rodríguez (Gl.12) & 23,022 & 0,133 & $-0,869$ & & 0,993 & $-0,0091$ & 0,1243 \\
\hline Hui (Gl. 13) & 372661,615 & $-10,012$ & 1,924 & & 0,987 & 0,5379 & 0,1669 \\
\hline Hui (Gl. 14) & 1,932 & & & & 0,987 & 0,5292 & 0,1669 \\
\hline $\begin{array}{c}\text { Messintervall } \\
\Delta \mathrm{t}=4 \text { Jahre }\end{array}$ & \multicolumn{4}{|c|}{ Geschätzte Modellparameter } & & \multicolumn{2}{|c|}{ Absolut } \\
\hline & a & $\mathrm{b}$ & c & $\mathrm{d}$ & Ryŷ & MRES & RMSE \\
\hline García (Gl.10) & 0,781 & & 5,286 & 5,828 & 0,995 & 0,0873 & 0,0655 \\
\hline Kvist Johannsen (Gl.11) & 0,642 & 0,465 & 0,007 & 0,045 & 0,995 & $-0,0027$ & 0,0651 \\
\hline Rodríguez (Gl.12) & 18,012 & 0,006 & $-0,725$ & & 0,996 & 0,0000 & 0,0608 \\
\hline Hui (Gl. 13) & 16,977 & $-14,448$ & 1,800 & & 0,989 & 0,5391 & 0,0955 \\
\hline Hui (Gl. 14) & 1,801 & & & & 0,989 & 0,5393 & 0,0954 \\
\hline $\begin{array}{l}\text { Messintervall } \\
\Delta \mathrm{t}=5 \text { Jahre }\end{array}$ & \multicolumn{4}{|c|}{ Geschätzte Modellparameter } & & \multicolumn{2}{|c|}{ Absolut } \\
\hline & a & $\mathrm{b}$ & $\mathrm{c}$ & $\mathrm{d}$ & Ryŷy & MRES & RMSE \\
\hline García (Gl.10) & 0,028 & & 5,078 & $-0,033$ & 0,996 & 0,0473 & 0,0685 \\
\hline Kvist Johannsen (Gl.11) & 74,037 & $-1,503$ & $-0,045$ & 0,037 & 0,992 & 0,0184 & 0,0922 \\
\hline Rodríguez (Gl.12) & 21,093 & 0,156 & $-0,903$ & & 0,996 & 0,0022 & 0,0669 \\
\hline Hui (Gl. 13) & 11,289 & 0,046 & 6,135 & & 0,989 & 0,3950 & 0,1080 \\
\hline Hui (Gl. 14) & 1,766 & & & & 0,984 & 0,7328 & 0,1268 \\
\hline
\end{tabular}

Tab. 7. Parameterwerte der Grundflächenmodelle sowie Absolut- und Relativwerte der Kriterien der Anpassungsgenanigkeit für drei-, vier-, und fünfjährige Messintervalle: Ryy = Korrelationskoeffizient; MRES = Mittelwert der Residuen; RMSE $=$ Wurzel des mittleren quadratischen Feblers.

Tab. 7. Values of the parameters as well as absolute and relative values of the criteria for analysing the model accuracy for time periods of three, four and five years: $R_{y y}=$ coefficient of correlation; MRES $=$ mean residual; RMSE = root mean square error.

Tabelle 8 und Abbildung 5 zeigen die nach Altersklassen stratifizierten Werte für MRES und RMSE. 


\begin{tabular}{|r|c|c|c|c|c|c|c|c|c|c|}
\hline & \multicolumn{5}{|c|}{ MRES } & \multicolumn{5}{c|}{ RMSE } \\
\hline $\begin{array}{r}\text { Alters- } \\
\text { bereich }\end{array}$ & $\begin{array}{c}\text { García } \\
\text { (Gl. 10) }\end{array}$ & $\begin{array}{c}\text { Johannsen } \\
\text { (Gl. 11) }\end{array}$ & $\begin{array}{c}\text { Rodríguez } \\
\text { (Gl. 12) }\end{array}$ & $\begin{array}{c}\text { Hui } \\
\text { (Gl. 13) }\end{array}$ & $\begin{array}{c}\text { Hui } \\
\text { (Gl. 14) }\end{array}$ & $\begin{array}{c}\text { García } \\
\text { (Gl. 10) }\end{array}$ & $\begin{array}{c}\text { Johannsen } \\
\text { (Gl. 11) }\end{array}$ & $\begin{array}{c}\text { Rodríguez } \\
\text { (Gl. 12) }\end{array}$ & $\begin{array}{c}\text { Hui } \\
\text { (Gl. 13) }\end{array}$ & $\begin{array}{c}\text { Hui } \\
\text { (Gl. 14) }\end{array}$ \\
\hline $20-30$ & $-0,0001$ & 0,0001 & $-0,0003$ & $-0,03461$ & $-0,03469$ & 0,0016 & 0,0025 & 0,0023 & 0,31278 & 0,31150 \\
\hline $30-40$ & 0,0004 & 0,0012 & 0,0006 & $-0,00577$ & $-0,00551$ & 0,0044 & 0,0062 & 0,0035 & 0,07697 & 0,07311 \\
\hline $40-50$ & $-0,0004$ & $-0,0003$ & $-0,0002$ & $-0,00826$ & $-0,00829$ & 0,0062 & 0,0046 & 0,0049 & 0,11026 & 0,10994 \\
\hline $50-60$ & 0,0000 & $-0,0003$ & $-0,0002$ & $-0,00091$ & $-0,00093$ & 0,0074 & 0,0097 & 0,0072 & 0,01218 & 0,01240 \\
\hline $60-70$ & $-0,0005$ & 0,0009 & $-0,0004$ & $-0,02330$ & $-0,02333$ & 0,0075 & 0,0076 & 0,0069 & 0,31091 & 0,30952 \\
\hline $70-80$ & 0,0011 & 0,0002 & 0,0005 & $-0,00134$ & $-0,00135$ & 0,0098 & 0,0085 & 0,0088 & 0,01783 & 0,01794 \\
\hline $80-90$ & 0,0007 & $-0,0004$ & 0,0003 & $-0,00283$ & $-0,00285$ & 0,0076 & 0,0058 & 0,0070 & 0,03781 & 0,03783 \\
\hline $90-100$ & 0,0003 & $-0,0005$ & $-0,0004$ & $-0,00694$ & $-0,00696$ & 0,0050 & 0,0068 & 0,0066 & 0,09257 & 0,09228 \\
\hline $100-110$ & 0,0009 & $-0,0004$ & 0,0002 & $-0,01474$ & $-0,01477$ & 0,0056 & 0,0040 & 0,0045 & 0,19669 & 0,19590 \\
\hline $110-120$ & 0,0002 & $-0,0006$ & $-0,0003$ & $-0,00832$ & $-0,00834$ & 0,0029 & 0,0045 & 0,0032 & 0,11106 & 0,11067 \\
\hline $120-130$ & 0,0009 & 0,0003 & 0,0003 & $-0,00013$ & $-0,00015$ & 0,0125 & 0,0067 & 0,0076 & 0,00176 & 0,00201 \\
\hline
\end{tabular}

Tab. 8. Nach Altersklassen stratifizierte Werte für MRES (a) und RMSE (b).

Tab. 8. MRES (a) and RMSE (b) according to age-classes.

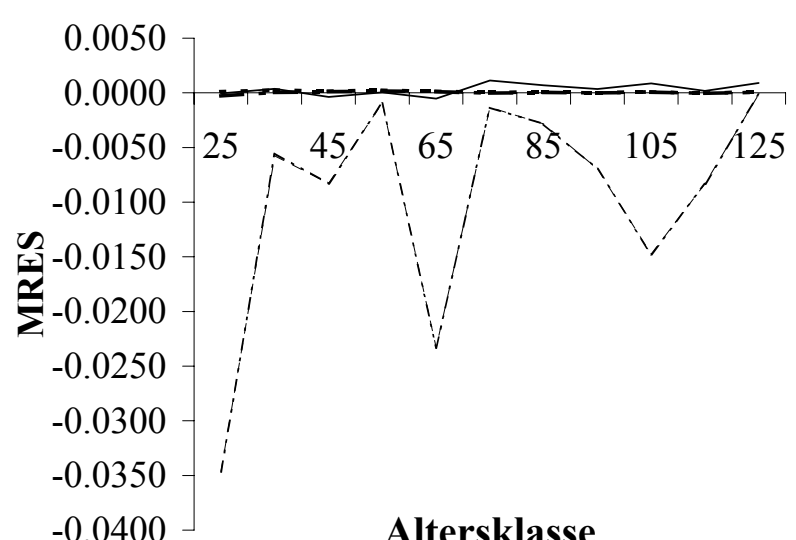

(a)

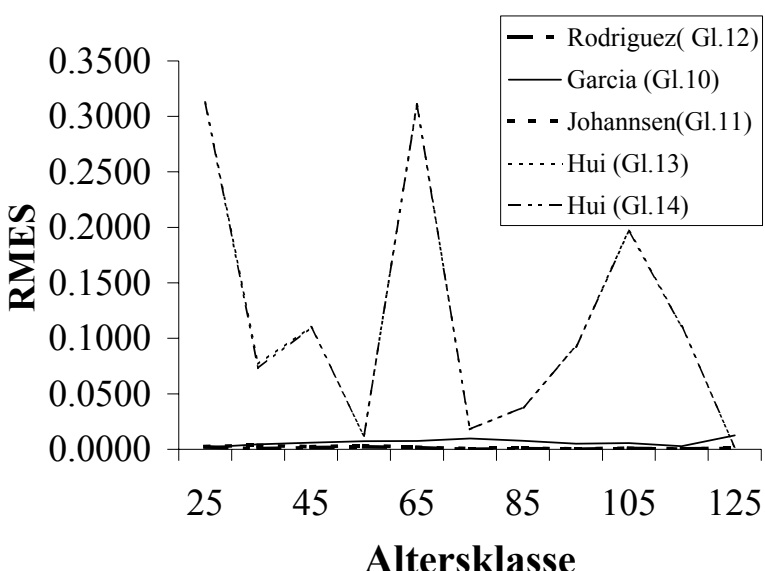

(b)

Abb. 5. Nach Altersklassen stratifizierte Werte für MRES (a) und RMSE (b).

Fig. 5. MRES (a) and RMSE (b) according to age-classes.

Der Vergleich zwischen den PID-Typ Modellen (13) und (14) und den in den PID-Typ umgewandelten Differentialgleichungen zeigt bessere Ergebnisse zu Gunsten der umgewandelten Gleichungen und von ihnen zeigt das Modell von Rodríguez die beste Anpassung.

Es wäre auch möglich den Vergleich auf der Differential-Ebene durchzuführen. Dazu wären die Gleichungen (13) und (14) in DIF-Modelle zu transformieren. Darauf wurde in der vorliegenden Arbeit verzichtet. Unsere Untersuchung hat gezeigt, dass es für die weniger gute Anpassung der DIF-Modelle im Vergleich zu den PID-Modellen eine mathematische Begründung gibt. Wie bereits erwähnt, nimmt der Korrelationskoeffizient zwischen zwei 
Variablen (z.B. $G_{2}$ und $G_{1}$ ) immer höhere Werte an als der Korrelationskoeffizient zwischen einer der beiden Variablen und der Differenz der Variablen.

\section{Schlussfolgerungen}

Die Untersuchung der Modelle des DIF-Typs hat die folgenden Ergebnisse gezeigt:

1. Der Vergleich der Modelle von García, Rodríguez und Kvist Johannsen nach dem Korrelationskoeffizienten (Ryŷ) zwischen Prognose- und beobachteten Werten, sowie der Wurzel des mittleren quadratischen Fehlers (RMSE) zeigt, dass das Modell von Rodríguez die beste Anpassung hat. Zur Berechnung der Modellparameter wurde die Methode der kleinsten Quadrate verwendet. Die Art der Anpassungsmethode hat hier vor allem bei Garcias Modell das Ergebnis beeinflusst.

2. Da die PID-Modelle die Grundfläche im Alter $t_{2}$ schätzen und die DIF-Modelle den laufenden Grundflächenzuwachs (Wachstumsrate), sind beide Gleichungstypen im Ergebnis nicht vergleichbar. Um den DIF- mit dem PID-Typ vergleichen zu können, wäre es nötig, die Differentialgleichungen von Rodríguez, García und Johannsen durch Integration oder numerisch zu lösen. Hier wurden lediglich die Approximationen solcher Integrale durch die PID-Modelle (10)-(12) ersetzt.

3. Aus der vergleichenden Analyse der oben genannten verarbeiteten Gleichungen und der PID-Modelle von Hui und Gadow (1993) und Hui (1997) ergibt sich, dass die in den PID-Typ umwandelten Modelle von García, Rodríguez und Johannsen eine bessere Anpassung zeigen und von ihnen das Modell von Rodríguez am besten ist.

\section{Danksagung}

Die Daten für diese Untersuchung wurden uns freundlicherweise von der NiEDERSÄCHSISCHEN FORSTLICHEN VERSUCHSANSTALT zur Verfügung gestellt. Dafür bedanken wir uns sehr herzlich. Für methodische Unterstützung und zahlreiche Hinweise danken wir Herrn Prof. Dr. Klaus v. Gadow ${ }^{2}$, Herrn Dr. Juha Lappi ${ }^{3}$, Herrn Prof. Dr. Dr. h. c. Branislav Sloboda ${ }^{4}$ und Herrn Prof. Dr. Joachim Saborowski ${ }^{5}$ sowie den Mitarbeitern im Institut für Forsteinrichtung und Ertragskunde, insbesondere Herrn Heydecke für seine Korrekturhinweise.

\footnotetext{
${ }^{2}$ Leiter des Instituts für Forsteinrichtung und Ertragskunde. Georg-August Universität Göttingen.

${ }^{3}$ METLA, Finnland.

${ }^{4}$ Leiter des Instituts für forstliche Biometrie und Informatik. Georg-August-Universität Göttingen.

${ }^{5}$ Institut für forstliche Biometrie und Informatik. Georg-August-Universität Göttingen.
} 


\section{Summary}

The purpose of this paper is to compare different stand level models for predicting the basal area annual increment of even aged stands of Norway spruce ( Picea abies L. Karst) in Northern Germany. The data (Tab. 1) collected to fit the models are derived from 87 permanent growth experiments which were provided by the NIEDERSÄCHSISCHEN FORSTLICHEN VERSUCHSANSTALT. The models tested in this paper belong to the differential equations form (Eq. 4-6) and between them the best performance (Tab. 3 and 4; Fig. 2) showed the model developed by Rodríguez (Eq. 6). Models belonging to the path invariant algebraic difference form (Eq. 13 and 14) were compared with the analysed differential equations. Since the first ones evaluate the basal area and the second ones the basal area growth rate it is not possible to compare both model types directly. The differential equation models had to be transformed into the path invariant algebraic difference form (Eq. 10-12). The transformed DIF models showed better performance (Tab. 7 and 8; Fig. 5) in Gprognosis as PID-models (Eq.13-14) and between them the model developed by RODRÍGUEZ showed the best results.

Keywords: Basal area; basal area increment; basal area modelling; Norway spruce stands.

\section{Résumé \\ Analyse de modèles de accroissement de surface terrière pour des peuplements équiennes d'épicéa}

Ce travail fait une étude comparative de différents modèles afin de prédire l'accroissement de la surface terrière en peuplements équiennes d'épicéa ( Picea abies L. Karst) situés dans le nord de l'Allemagne. Les données utilisées pour calculer les paramètres des modèles (Tab. 1) proviennent de 87 parcelles d'essai de peuplements équiennes d'épicéa et elles ont été prises par le NIEDERSÄCHSISCHEN FORSTLICHEN VERSUCHSANSTALT. Les modèles analysés dans cette communication appartiennent au type d'équations différentielles (Eq. 4-6); entre eux le modèle développé par Rodríguez (Eq. 6) a donné le résultat plus satisfaisant (Tab. 3 et 4; Fig. 2). Les models qui appartiennent au type de projection invariable de différence algébrique (Eq. 13-14) ont été comparés avec les modèles des équations différentielles analysés. Puisque les premières évaluent la surface terrière et les deuxièmes l'accroissement de la surface terrière il n'est pas possible de comparer directement tous les deux types de modèles. Les modèles des équations différentielles ont été transformés en type de projection invariable de différence 
algébrique (Eq. 10-12). Ces modèles transformés ont donné un meilleur ajustement et entre eux, le modèle développé par Rodríguez (Eq. 12) a donné le résultat plus satisfaisant (Tab. 7 et 8; Fig. 5).

\section{Literatur}

Alder, D., 1980: Estimación del volumen forestal y predicción del rendimiento. Estudios FAO. Montes 22/2, Roma.

Álvarez, J.G., 1997: Análisis y caracterización de las distribuciones diamétricas de Pinus pinaster Ait. en Galicia. Diss. Uni. Madrid, 270 S.

Assmann, E., 1961: Waldertragskunde. BLV Verlagsgesellschaft, München-Bonn-Wien, 490 $\mathrm{S}$.

Clutter, J.L., 1963:Compatible growth and yield models for loblolly pine. For. Sci. 9:354-371.

Clutter, J.L. und Lenhart, J.D., 1971: Cubic foot yield tables for old field loblolly pine plantations in the Georgia Piedmont. Ga. For. Res. Council, Report 22 Series 3.

Forss, E., 1994: Das Wachstum der Baumart Acacia mangium in Südkalimantan, Indonesien. Magister Diss., Fac. Of Forestry, Univ. of Göttingen, 87 S.

Gadow, K. v., 1992: Wachstums- und Ertragsmodelle für die Forsteinrichtung. Bericht zur Jahrestagung des Deutschen Verbandes Forstlicher Forschungsanstalten, Sektion Ertragskunde, Jahrestagung in Grillenburg.

Gadow, K. v. und Hui, G. Y., 1993: Stammzahlentwicklung und potentielle Bestandesdichte bei Cunninghamia lanceolata. Centralblatt für das gesamte Forstwesen 110 (2): 41-48.

Gadow, K. v. und Hui, G. Y., 1999: Modelling forest development. Kluwer, 213 S.

García, O., 1994: The state space approach in growth modelling. Can. J. For. Res. 24: 18941903.

Gurjanov, M., Sánchez Orois, S. und Schröder, J., 2000: Grundflächenmodelle für gleichaltrige Fichtenreinbestände. Eine vergleichende Analyse. Centralblatt für das gesamte Forstwesen (im Druck).

Hradetzky, J., 1972: Modell eines integrierten Ertragstafel-Systems in modularer Form. Mitteilungen der Baden-Württembergischen Forstlichen Versuchs- und Forschungsanstalt, $169 \mathrm{~S}$.

Hui, G. Y., 1997: Wuchsmodelle für die Baumart Cunninghamia lanceolata. Cuvillier Verlag Göttingen, $121 \mathrm{~S}$.

Hui, G. Y. und Gadow K. v., 1993: Zur Modellierung der Bestandesgrundflächenentwicklung - dargestellt am Beispiel der Baumart Cunninghamia lanceolata. Allgemeine Forst- und Jagdzeitung 164: 144-149.

Kvist Johannsen, V., 1999: A growth model for oak in Denmark. Ph. D. Dissertation, Danish Forest and Landscape Research Institute, Hørsholm, Denmark, 197 S.

Lappi, J., 1993: Metsäbiometrian menetelmiä. Silva Carelica 24, 190 S.

Nagel, J. und Biging G. S., 1995: Schätzung der Parameter der Weibull-Funktion zur Generierung von Durchmesserverteilungen. Allgemeine Forst- und Jagdzeitung 199 (9/10):185-189.

Pretzsch, H., 1992: Konzeption und Konstruktion von Wuchsmodellen für Rein- und Mischbestände. Forstliche Forschungsberichte München, No. 115.

Rodríguez, R., 1995: Crecimiento y producción de masas forestales regulares de Pinus pinaster Ait. en Galicia. Alternativas selvícolas posibles. Ph. D. Dissertation, Escuela Técnica Superior de Ingenieros de Montes. Madrid, 297 S. 
Schübeler, D., 1997: Untersuchungen zur standortabhängigen Wachstumsmodellierung bei der Fichte. Dissertation zur Erlangung des Doktorgrades der Forstlichen Fakultät der Georg-August-Universität in Göttingen, 155 S.

Schumacher, F.X., 1939: A new growth curve and its applications to timber-yield studies. Journal of Forestry 37:819-820.

Sloboda, B., 1971: Zur Darstellung von Wachstumsprozessen mit Hilfe von Differentialgleichungen erster Ordnung. Inaugural-Dissertation zur Erlangung der Doktorwürde der Forstwissenschaftlichen Fakultät der Albert-Ludwings-Universität zu Freiburg im Breisgau, 109 S.

Souter, R. A., 1986: Dynamic stand structure in thinned stands of naturally regenerated loblolly pine in the Georgia Piedmont. Ph. D. Thesis. University of Georgia, Athens, GA.

Staupendahl, K., 1999: Modelling thinnings based on the ratio of relative removal rates. Growth and yield modelling of tree plantations in south and east Africa. University of Joensuu, S. 183.

Wenk, G., Antanaitis, V. und Smelko, S., 1990: Waldertragslehre. Deutscher Landwirtschaftsverlag. Berlin. 


\section{Veröffentlichung III}

Bewertung und Optimierung von Vornutzungen in Fichtenbeständen

SÁNCHEZ OROIS, S. UND VILČKO, F.

Erschienen in:

Forstwissenschaftliches Centralblatt. Jahrgang 2002, 121:250-264. 


$$
\text { . }
$$




\title{
Bewertung und Optimierung von Vornutzungen in Fichtenbeständen
}

Evaluation and optimization of the thinning phase in spruce stands

\author{
SOFÍA SÁNCHEZ OROIS UND FRANTIŠEK VILČKO
}

\begin{abstract}
Institut für Waldinventur und W aldwachstum. Georg-August-Universität Göttingen Büsgenweg 5, 37077 Göttingen. Germany
\end{abstract}

\section{Zusammenfassung}

Das Ziel der Untersuchung ist die Integration der Einzel- und Gesamtvornutzungsplanung am Beispiel eines Waldes mit 38 Fichtenbeständen. Die Lösung dieses Problems erfordert die Generierung, Bewertung und schließlich die Optimierung von Managementoptionen. Zur Generierung der Managementoptionen wurde ein Bestandeswuchsmodell und ein Durchforstungsalgorithmus verwendet, der es ermöglicht, forstliche Begriffe wie Stärke und Art der Durchforstung in eine quantitative Entnahmevorschrift zu übertragen. Mit Hilfe des Managementsimulators Jakta wurden zahlreiche Managementalternativen für die einzelnen Bestände generiert. Bei der anschließenden Bewertung der Optionen wurde das ökonomische Kriterium Bestandeserwartungswert (BEW) verwendet. Die Optimierung befasst sich mit der Evaluierung multipler Waldentwicklungen für einen Forstbetrieb, d.h. mit der Bestimmung des optimalen Raum-Zeit-Musters von Durchforstungsmaßnahmen für den ganzen Forstbetrieb. Zu diesem Zweck wurde das Softwarepaket JLP verwendet. Schließlich wurde eine Sensitivitätsanalyse für die optimale Lösung unter Berücksichtigung von Änderungen des Holzpreises und der Zinsrate durchgeführt.

Schlagwörter: Forstplanung, Optimierung, Generierung, Managementoptionen, Bestandeserwartungswert, Bewertung, Fichtenbestände. 


\section{Einleitung}

Die Planung der multifunktionalen, nachhaltigen Waldnutzung ist im deutschen Sprachraum seit etwa Mitte des 18. Jahrhunderts bekannt als Forsteinrichtung. Aufgabe der Forsteinrichtung ist die Zustandserfassung und Zustandsbeschreibung, die Planung der Waldnutzung unter Berücksichtigung vielfältiger Ansprüche und Beschränkungen und die Kontrolle der Waldentwicklung. Auf der Basis waldwachstumskundlicher Erkenntnisse und mit spezifischen Ansätzen aus dem Bereich der Unternehmensforschung können die Auswirkungen unterschiedlicher Optionen der Waldentwicklung generiert und bewertet werden (Gadow, 2001).

Bei der Forsteinrichtung handelt es sich, wie bei anderen betrieblichen Planungen, um die Bereitstellung entscheidungsrelevanter Informationen. Dennoch ist die Forsteinrichtung mit erschwerten Bedingungen konfrontiert. Zum Beispiel gibt es verschiedene und manchmal widersprüchliche Ziele (z.B. Maximierung der Nettoerträge, Maximierung der nachhaltigen Nutzung, Erhaltung der Biodiversität). Außerdem wird die Planung auf Grund der Komplexität der Steuerung der Waldökosysteme erheblich erschwert. Schließlich erfordert eine realistische Forsteinrichtung die Vorausschau über lange Zeitperioden um die langfristigen Auswirkungen forstlicher Maßnahmen realistisch abzubilden (Öhman, 2001).

Ein zentrales Problem der Forsteinrichtung ist die Integration der Einzelplanung (auf Bestandesebene) und der Gesamtnutzungsplanung für den ganzen Forstbetrieb (Gadow, 1991). Ein Bestand ist eine Einheit eines Forstbetriebes mit definierten Grenzen; benachbarte Bestände können sehr unterschiedliche Waldbestockungen aufweisen. Die Einzelplanung befasst sich mit der Bestimmung der Bestandeseingriffe. Die Gesamtplanung bestimmt die beste Managementoption für jeden Bestand unter Berücksichtigung gesamtbetrieblicher Beschränkungen (Wikström, 2000). Die Forsteinrichtung hat zum Ziel, die Nachhaltigkeit der verschiedenen Funktionen des Waldes zu überwachen und gleichzeitig die waldbaulichen und wirtschaftlichen Ziele der Betriebsleitung zu unterstützen. Das Resultat ist ein Plan, der die einzelnen erwünschten Bestandesentwicklungen und die Gesamtnutzung des Betriebes optimal in Einklang bringt.

Weiterhin entscheidend für die forstbetriebliche Steuerung ist die Generierung von Optionen. Ein Simulationsmodell bietet die Möglichkeit, unterschiedliche waldbauliche Maßnahmen für einen realen Bestand zu simulieren. Eine Eingriffsfolge wird durchgespielt, 
bevor sie im Wald umgesetzt wird. Wachstumssimulatoren werden heute nicht nur zur kurzfristigen Prognose bei der Fortschreibung von gespeicherten Inventurdaten eingesetzt, sondern dienen vor allem auch zur Simulation unterschiedlicher Waldentwicklungspfade in realen oder hypothetischen Beständen (Rodríguez Soalleiro, 1995; Gadow u. Hui, 1999). Häufig sind mehrere gleichwertige Alternativen für einen gegebenen Ausgangszustand denkbar und daher ist es einleuchtend, wenn unterschiedliche mögliche Waldentwicklungsoptionen analysiert und bewertet werden. Die Auswahl der optimalen Variante für einen speziellen Bestand wird vor allem von den gegenwärtigen Zustandsgrößen, von der Gewichtung der unterschiedlichen Zielsetzungen und von den gesamtbetrieblichen Beschränkungen bestimmt. Entscheidend für die betriebliche Steuerung ist daher die Örtlichkeit der Information.

Ziel der vorliegenden Arbeit ist die Generierung, Bewertung und anschließend die Optimierung von Managementoptionen für die Vornutzungsphase von Fichtenbeständen. Zur Generierung der Managementoptionen wurde der Managementsimulator Jakta (Lappi, 2002) verwendet. Anschließend wurde die Optimierung nach ökonomischen Kriterien mit Hilfe des von Lappi (1992) entwickelten Softwaresystems JLP durchgeführt.

\section{Daten und Methoden}

Die für diese Untersuchung verwendeten Daten stammen aus 38 Fichtenbeständen des Waldteiles Winnefeld Süd in Solling. In Tabelle 1 sind die in dieser Untersuchung verwendeten Anfangsdaten für jeden Bestand zusammengefasst:

\begin{tabular}{|c|c|c|c|c|c|c|c|c|}
\hline $\begin{array}{c}\text { Alter } \\
\text { (Jahre) }\end{array}$ & Abt & Uabt & $\begin{array}{c}\text { Fläche } \\
(\mathrm{ha})\end{array}$ & $\begin{array}{c}\mathrm{H} \\
(\mathrm{m})\end{array}$ & $\begin{array}{c}\text { SI } \\
(\mathrm{m})\end{array}$ & $\begin{array}{c}\mathrm{Dg} \\
(\mathrm{cm})\end{array}$ & $\begin{array}{c}\mathrm{G} \\
\left(\mathrm{m}^{2} \mathrm{ha}^{-1}\right)\end{array}$ & $\begin{array}{c}\mathrm{N} \\
\text { pro ha }\end{array}$ \\
\hline 7 & 219 & $\mathrm{e}$ & 2.2 & 0.0 & 0 & 2 & 0 & 6010 \\
\hline 7 & 221 & $\mathrm{c} 3$ & 1.3 & 0.0 & 0 & 2 & 0 & 6050 \\
\hline 7 & 221 & $\mathrm{~d}$ & 3.5 & 0.0 & 0 & 2 & 0 & 6020 \\
\hline 7 & 222 & $\mathrm{~b} 2$ & 4.0 & 0.0 & 0 & 2 & 0 & 6030 \\
\hline 14 & 220 & $\mathrm{~b} 2$ & 0.7 & 0.0 & 0 & 4 & 0 & 5200 \\
\hline 15 & 219 & $\mathrm{~d} 2$ & 0.7 & 0.0 & 0 & 4 & 0 & 5050 \\
\hline 17 & 219 & $\mathrm{a} 2$ & 0.4 & 0.0 & 0 & 5 & 0 & 5000 \\
\hline 17 & 220 & $\mathrm{c}$ & 2.5 & 0.0 & 0 & 6 & 0 & 5000 \\
\hline 17 & 223 & $\mathrm{a}$ & 1.9 & 0.0 & 0 & 5 & 0 & 5000 \\
\hline 23 & 219 & $\mathrm{~d} 1$ & 2.1 & 11.4 & 40 & 9 & 27 & 4244 \\
\hline 23 & 220 & $\mathrm{~b} 1$ & 1.3 & 13.8 & 43 & 10 & 32 & 4074 \\
\hline 27 & 225 & $\mathrm{~b}$ & 5.0 & 13.8 & 39 & 10 & 32 & 4074 \\
\hline 29 & 224 & $\mathrm{a}$ & 5.0 & 16.5 & 41 & 12 & 34.1 & 3015 \\
\hline 32 & 226 & $\mathrm{~d}$ & 4.3 & 16.5 & 38 & 12 & 30.6 & 2706 \\
\hline 37 & 224 & $\mathrm{~b} 2$ & 1.9 & 17.9 & 37 & 13 & 30.4 & 2290 \\
\hline
\end{tabular}




\begin{tabular}{|c|c|c|c|c|c|c|c|c|}
\hline $\begin{array}{c}\text { Alter } \\
\text { (Jahre) }\end{array}$ & Abt & Uabt & $\begin{array}{c}\text { Fläche } \\
(\text { ha })\end{array}$ & $\begin{array}{c}\text { H } \\
(\mathrm{m})\end{array}$ & $\begin{array}{c}\text { SI } \\
(\mathrm{m})\end{array}$ & $\begin{array}{c}\text { Dg } \\
(\mathrm{cm})\end{array}$ & $\begin{array}{c}\text { G } \\
\left(\mathrm{m}^{2} \mathrm{ha}^{-1}\right)\end{array}$ & $\begin{array}{c}\text { N } \\
\text { pro ha }\end{array}$ \\
\hline 38 & 225 & $\mathrm{a}$ & 4.2 & 21.9 & 40 & 16 & 32.9 & 1636 \\
\hline 40 & 220 & $\mathrm{~d}$ & 7.2 & 21.9 & 39 & 16 & 36.6 & 1820 \\
\hline 40 & 222 & $\mathrm{a}$ & 9.0 & 21.9 & 39 & 16 & 32.9 & 1636 \\
\hline 41 & 221 & $\mathrm{~b} 1$ & 5.3 & 20.4 & 37 & 15 & 31.6 & 1788 \\
\hline 41 & 223 & $\mathrm{~b} 1$ & 3.4 & 21.9 & 39 & 16 & 36.6 & 1820 \\
\hline 41 & 223 & b2 & 3.8 & 21.9 & 39 & 16 & 32.9 & 1636 \\
\hline 42 & 221 & $\mathrm{c} 2$ & 1.2 & 21.9 & 38 & 16 & 36.6 & 1820 \\
\hline 44 & 221 & b2 & 2.8 & 24.4 & 40 & 18 & 22.7 & 892 \\
\hline 44 & 226 & a & 3.7 & 24.4 & 40 & 18 & 34.1 & 1340 \\
\hline 45 & 221 & a & 7.2 & 24.4 & 39 & 18 & 41.6 & 1635 \\
\hline 46 & 224 & b1 & 4.7 & 22.8 & 37 & 17 & 36.8 & 1621 \\
\hline 47 & 219 & c & 6.9 & 22.8 & 37 & 17 & 36.8 & 1621 \\
\hline 50 & 222 & c & 2.0 & 24.9 & 38 & 19 & 42.2 & 1488 \\
\hline 50 & 223 & c & 1.3 & 24.9 & 38 & 19 & 38.4 & 1354 \\
\hline 52 & 219 & a1 & 4.5 & 24.9 & 37 & 19 & 30.7 & 1083 \\
\hline 63 & 219 & a3 & 4.6 & 29.5 & 38 & 25 & 47.4 & 966 \\
\hline 65 & 220 & b3 & 0.6 & 28.1 & 36 & 23 & 25.1 & 604 \\
\hline 68 & 226 & b & 5.0 & 29.2 & 36 & 25 & 25.8 & 526 \\
\hline 68 & 226 & c & 2.3 & 29.2 & 36 & 25 & 30.1 & 613 \\
\hline 72 & 219 & b & 2.8 & 24.8 & 30 & 36 & 28.1 & 276 \\
\hline 83 & 220 & a & 2.0 & 28.0 & 31 & 39 & 25.6 & 204 \\
\hline 86 & 221 & c1 & 0.7 & 28.8 & 31 & 26 & 29.3 & 552 \\
\hline 116 & 222 & b1 & 1.8 & 34.6 & 32 & 38 & 34.6 & 305 \\
\hline & & Summe & 129.6 & & & & & \\
\hline
\end{tabular}

Tabelle 1. Anfangsdaten für 38 Fichtenbestände des Waldteiles Winnefeld Süd. Abt: Abteilung; Uabt: Unterabteilung; H: Oberhöhe; SI: Site index; Dg: Durchmesser des Grundflächenmittelstammes; G: Grundfläche; N: Stammzahl.

Table 1. Initial data for 38 spruce stands of the forest Winnefeld Süd. Abt: Compartment; Uabt: Subcompartment; H: Dominant height; SI: Site index; Dg: Quadratic mean diameter; G: Basal area, $N$ : Stem number.

Die Fläche des untersuchten Waldteiles beträgt insgesamt 129.6 ha. Voraussetzung für die Generierung von Managementoptionen ist das Vorhandensein eines Wachstums- und Durchforstungsmodells.

\subsection{Das Wuchsmodell}

Das für diese Untersuchung verwendete Fichtenwuchsmodell wurde von Vilčko und Gadow (2002) auf Basis der Arbeiten von Schübeler (1997), Gurjanov et al. (2001) und Sánchez et al. (2001) entwickelt. Tabelle 2 zeigt die für die Berechnung der wichtigsten Bestandesgrößen verwendeten Funktionen. 


\begin{tabular}{|l|l|}
\hline Grundfläche & $G_{2}=\exp \left(\left(\frac{A_{1}}{A_{2}}\right) \cdot \ln G_{l}+5.571869 \cdot\left(1-\frac{A_{1}}{A_{2}}\right)-0.011896 \cdot S I \cdot\left(1-\frac{A_{l}}{A_{2}}\right)-0.009004 \cdot\left(\ln N_{2}-\left(\frac{A_{l}}{A_{2}}\right) \cdot \ln N_{l}\right)\right)$ \\
\hline Oberhöhe & $H_{100}=97.6623 \cdot\left(\frac{S I}{97.6623}\right)^{\exp \left(\frac{-1.782423}{(1.288161-1) \cdot A_{100}^{(1.288161-1)}}+\frac{1.782423}{(1.288161-1) \cdot A^{(1.288161-1)}}\right)}$ \\
\hline Mittelhöhe & $H_{m}=0.610260 \cdot H^{1.123689}$ \\
\hline Volumen & $V=\left[0.72674+0.499289 \cdot\left(H_{m}\right)-0.002097 \cdot\left(H_{m}{ }^{2}\right)\right] \cdot G$ \\
\hline Mortalität & $N_{2}=1000 \cdot\left[\left(\frac{N_{1}}{1000}\right)^{-0.543767}+0.000305 \cdot\left(H_{2}^{2.2527}-H_{1}^{2.2527}\right)\right]^{\frac{1}{-0.543767}}$ \\
\hline
\end{tabular}

Tabelle 2. Grundlegende Funktionen des von Vilckeo und Gadow (2002) entwickelten Fichtenwuchsmodells.

Table 2. Basic functions of the growth model developed by Vilckeo and Gadow (2002)

Die Koeffizientenschätzungen wurden mit Hilfe des statistischen Programmpakets STATISTICA nach der nichtlinearen Methode der kleinsten Quadrate durchgeführt.

Die erwähnten Komponenten des Wuchsmodells ermöglichen eine Schätzung des Bestandeswachstums zwischen zwei Durchforstungen.

\subsection{Das Durchforstungsmodell}

Eine Durchforstung lässt sich vereinfacht durch die Faktoren Durchforstungsstärke, Durchforstungsart und Zeitpunkt des Eingriffes charakterisieren. Zum Beispiel kann die Durchforstungsstärke durch das Verhältnis der ausscheidenden Grundfläche zur Grundfläche des Gesamtbestandes bzw. durch die Änderung des Bestockungsgrades (Kramer, 1990) beschrieben werden. Spellmann et al. (1999) schlagen drei Pflegephasen für Fichtenbestände vor. Eine Durchforstung wird durchgeführt, wenn die Oberhöhe sich innerhalb eines vorgegebenen Intervalls befindet und gleichzeitig der aktuelle Bestockungsgrad größer als der Zielbestockungsgrad, definiert als Anteil vom natürlichen Bestockungsgrad, ist. In Tabelle 3 sind die Durchforstungskriterien für jede Pflegephase zusammengefasst. 


\begin{tabular}{|c|c|c|c|}
\hline Pflegephase & $\boldsymbol{H}_{100}$ & $\boldsymbol{B}_{\text {ziel }}^{\circ}$ & $\boldsymbol{G}_{\text {maxent }}$ \\
\hline $\mathbf{1}$ & $14-20 \mathrm{~m}$ & 0.70 & $\left(G_{\text {vor }} / G_{\max }-0.7\right) \cdot G_{\max }$ \\
\hline $\mathbf{2}$ & $20-26 \mathrm{~m}$ & 0.75 & $\left(G_{\text {vor }} / G_{\max }-0.75\right) \cdot G_{\max }$ \\
\hline $\mathbf{3}$ & $>26 \mathrm{~m}$ & 0.80 & $\left(G_{\text {vor }} / G_{\max }-0.8\right) \cdot G_{\max }$ \\
\hline
\end{tabular}

Tabelle 3. Durchforstungskeriterien für die 3 Pflegephasen und Gleichungen zur Berechnung des maximalen Nutzungspotentials. $H_{100}=$ Oberböhe; $\quad B_{\text {ziel }}^{\circ}=$ Zielbestockungsgrad; $G_{\text {maxent }}=$ maximal entnommene Grundfläche; $G_{v o r}=$ Grundfläche des Gesamtbestandes vor dem Eingriff, $G_{\max }=$ maximale Grundfläche (natürlicher Bestockungsgrad).

Table 3. Thinning criteria for the 3 phases and the equations for the assessment of the maximum potential use. $H_{100}=$ Dominant height; $B_{z i e l}^{\circ}=$ Stand density; $G_{\text {maxent }}=$ maximum harvested basal area; $G_{v o r}=$ Basal area before thinning; $G_{\max }=$ maximum basal area.

Bei der Herleitung des maximalen Nutzungspotentials wurde von folgender Definition des Zielbestockungsgrads $\left(\mathrm{B}_{\text {ziel }}^{\circ}\right)$ ausgegangen:

$$
B_{z i e l}^{\circ}=\frac{G_{z i e l}}{G_{\max }}
$$

Zur Berechnung der maximalen Grundflächen wurde die von Döbbeler und Spellmann (2002) parametrisierte Gleichung verwendet:

$$
G_{\max }=\frac{\pi}{160000 \cdot 1.28745 \cdot 10^{-6} \cdot 1.2842 \cdot H_{100}^{(0.7148-1.1914)}}
$$

wobei:

$$
\begin{aligned}
& \mathrm{H}_{100}=\text { Oberhöhe }(\mathrm{m}) ; \\
& \mathrm{G}_{\max }=\text { Maximale Grundfläche }\left(\mathrm{m}^{2} \mathrm{ha}^{-1}\right) .
\end{aligned}
$$

Ähnlich wie der Zielbestockungsgrad lässt sich die aktuelle Bestockungsgrad (Bvor ${ }^{\circ}$ ) als auch die entnommene Bestandesdichte $\left(\mathrm{B}_{\mathrm{ent}}{ }^{\circ}\right)$ definieren:

$$
\begin{aligned}
& B_{\text {vor }}^{\circ}=\frac{G_{\text {vor }}}{G_{\max }} \\
& B_{\max e n t}^{\circ}=\frac{G_{\max e n t}}{G_{\max }}
\end{aligned}
$$

wobei:
$\mathrm{B}_{\text {vor }}{ }^{\circ} \quad=$ Bestockungsgrad des Gesamtbestandes vor dem Eingriff;
$\mathrm{G}_{\mathrm{vor}} \quad=$ Grundfläche des Gesamtbestandes vor dem Eingriff $\left(\mathrm{m}^{2} \mathrm{ha} \mathrm{a}^{-1}\right)$;
$\mathrm{G}_{\max } \quad=$ Maximale Grundfläche $\left(\mathrm{m}^{2} \mathrm{ha}^{-1}\right)$;
$\mathrm{G}_{\text {max ent }}=$ Maximal entnommene Grundfläche $\left(\mathrm{m}^{2} \mathrm{ha} \mathrm{a}^{-1}\right)$;
$\mathrm{B}_{\text {ent }}{ }^{\mathrm{o}} \quad=$ Bestockungsgrad des entnommenen Bestandes. 
Falls der aktuelle Bestockungsgrad größer als der Zielbestockungsgrad ist, kann eine Durchforstung durchgeführt werden. Der maximal zu entnehmende Grundflächenanteil wird dann wie folgt hergeleitet:

$$
\begin{aligned}
& B_{v o r}^{\circ}-B_{\max e n t}^{\circ}=B_{z i e l}^{\circ} \Leftrightarrow \frac{G_{v o r}}{G_{\max }}-\frac{G_{\max e n t}}{G_{\max }}=B_{z i e l}^{\circ} \\
& G \max e n t=\left(G v o r / G \max -B_{z i e l}^{o}\right) \cdot G \max
\end{aligned}
$$

wobei:

$$
\begin{array}{ll}
B_{\text {ziel }}{ }^{\circ} & =\text { Zielbestockungsgrad; } \\
G_{\text {ziel }} & =\text { Zielgrundfläche; } \\
B_{\text {vor }}{ }^{o} & =\text { Bestockungsgrad des Gesamtbestandes vor dem Eingriff; } \\
G_{v o r} & =\text { Grundfläche des Gesamtbestandes vor dem Eingriff }\left(\mathrm{m}^{2} \mathrm{ha}^{-1}\right) ; \\
B_{\text {ent }}{ }^{-1} & =\text { Bestockungsgrad des ausscheidenden Bestandes; } \\
G_{\text {maxent }} & =\text { Maximal entnommene Grundfläche }\left(\mathrm{m}^{2} \mathrm{ha}^{-1}\right) ; \\
G_{\text {max }} & =\text { Maximale Grundfläche }\left(\mathrm{m}^{2} \mathrm{ha}^{-1}\right) .
\end{array}
$$

Neben der Eingriffsstärke ist die Eingriffsart der zweite Faktor, der die weitere Bestandesentwicklung beeinflusst. Die Art des Eingriffs wird durch die selektive Entnahme ausgewählter Teile der Population bestimmt und kann z.B. mit Hilfe des Quotienten aus relativer Stammzahl- und relativer Grundflächenentnahme NG beschrieben werden (Kassier, 1993; Staupendahl, 1999; Staupendahl u. Puumalainen, 1999)

$$
N G=\frac{N_{\text {ent }} / N}{G_{\text {ent }} / G}=\frac{r N}{r G}
$$

Wobei:

$$
\begin{array}{ll}
N_{\text {ent }} & =\text { Entnommene Stammzahl; } \\
N & =\text { Gesamte Stammzahl; } \\
G & =\text { Gesamte Grundfläche; } \\
G_{\text {ent }} & =\text { Entnommene Grundfläche. }
\end{array}
$$

Die Gleichungen 7 und 8 schätzen den NG-Parameter für zwei Durchforstungsarten: Hochdurchforstung und Niederdurchforstung (Vilčko, 2002).

$$
\begin{aligned}
& N G_{H D f}=1.1+0.18 \cdot \ln (D g) \\
& N G_{N D f}=4-0.68 \cdot \ln (D g)
\end{aligned}
$$

Wobei:

$D g \quad=$ Durchmesser des Grundflächenmittelstamms (cm);

$N G_{H D}=N G$-Verhältnis für Hochdurchforstung;

$N G_{N D}=N G$-Verhältnis für Niederdurchforstung. 
Auf der Basis des beschriebenen Wachstums- und Durchforstungsmodells wurden die Managementoptionen mit Hilfe des Simulators Jakta generiert.

\subsection{Jakta: ein Simulator zur computergestützten Generierung von Managementoptionen}

Das statistische Auswertungsprogramm Jakta wurde von Lappi (2002) entwickelt. Zusätzlich zu dem Simulator zur Generierung von Managementoptionen stehen statistische Anwendungen zur Verfügung. Das Wachstums- und Durchforstungsmodell wurde in JaktaCode implementiert. Notwendige zusätzliche Angaben sind die Anzahl der Jahre innerhalb einer Planungsperiode (k) und die Anzahl der Planungsperioden (s). In unserem Fall ist $\mathrm{k}=5$ Jahre und s $=4$; der Planungshorizont beträgt also 4 × $5=20$ Jahre.

Die Anzahl der generierten Optionen für einen Bestand hängt von der Anzahl der Planungsperioden, von der Art und Stärke der Durchforstung und vom Planungshorizont ab. Zur Vereinfachung des Simulationsmodells wurden in unserem Beispiel nur Niederdurchforstungen simuliert, in welchen immer eine mäßige Durchforstung durchgeführt wurde. Unter Berücksichtigung, dass für einen Bestand zwei mögliche Behandlungsoptionen existieren (Durchforstung oder keine Durchforstung) und dass insgesamt die ganze Simulation vier 5-jährige Planungsperioden umfasst, beträgt die Anzahl der Managementoptionen pro Bestand 24. Abbildung 1 stellt die von Jakta generierten möglichen Optionen dar.

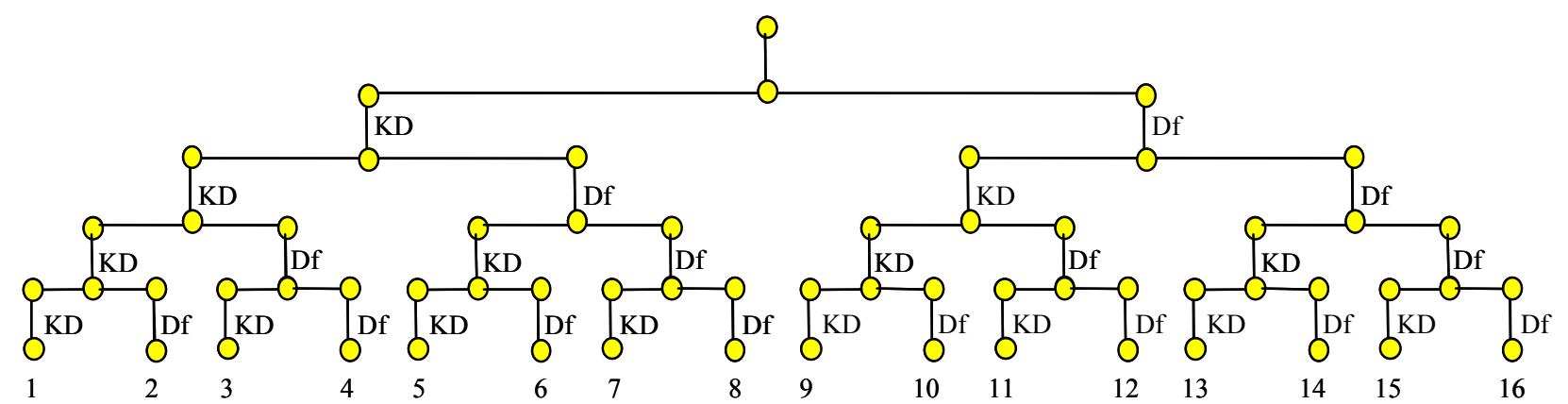

Abb. 1. Anzabl aller möglichen Optionen für einen Bestand. Df: Durchforstung, KD: Keine Durchforstung.

Fig. 1. All possible management options for one stand. Df: Thinning, KD: No thinning.

Die theoretische Anzahl möglicher Optionen wird durch die Durchforstungskriterien beschränkt, da aufgrund des jungen Bestandesalters oder des niedrigen Bestockungsgrades nicht immer eine Durchforstung pro Planungsperiode möglich bzw. erforderlich ist. 


\subsection{Bewertung der Managementoptionen}

Aufgrund der Multifunktionalität der Forstwirtschaft können die Managementoptionen nach verschiedenen Kriterien bewertet werden. In diesem Beispiel wurde eine ökonomische Bewertung mit Hilfe der Formel zur Berechnung des Bestandeserwartungswertes (BEW) durchgeführt. In zukünftigen Arbeiten wäre es auch interessant, weitere Kriterien zur Bewertung der Optionen zu berücksichtigen (z.B. die Förderung der Diversität oder die Minimierung der Schwankungen des periodischen Erntevolumens).

Der diskontierte Jetztwert einer Eingriffsfolge ergibt sich aus der Summe der diskontierten Reinerträge, dem diskontierten Terminalwert und dem diskontierten Bodenerwartungswert.

$$
B E W_{t-t_{0}}=\frac{\sum_{i=t_{0}}^{t} R E_{i} e^{r\left(t-t_{i}\right)}}{e^{r\left(t-t_{0}\right)}}+\frac{T_{t}}{e^{r\left(t-t_{0}\right)}}+\frac{B_{t}}{e^{r\left(t-t_{0}\right)}}
$$

wobei:

$$
\begin{array}{ll}
B E W= & \text { Bestandeserwartungswert; } \\
t & =\text { Alter, in dem ein Durchmesser von mindestens } 40 \mathrm{~cm} \text { erreicht wird; } \\
t_{0} & =\text { Bestandesalter am Anfang der Planungsperiode; } \\
\mathrm{RE}_{i}= & \text { Reinerträge im Jahr } i, \mathrm{~d} . \text { h. alle Einzahlungen minus alle Auszahlungen zum } \\
& \text { Zeitpunkt } i ; \\
T_{t} & =\text { erntekostenfreier Terminalwert des Bestandes zum Zeitpunkt } t \\
r & =\text { Zinsrate }(\mathrm{r}=4 \%) ; \\
B_{t} & =
\end{array}
$$

Diese Formel ist eine Weiterentwicklung der sog. Zeitfenstermethode der dynamischen Investitionsrechnung für Dauerwaldsysteme (Hille et al., 1999). Die Besonderheit der Gl. (9) liegt in der Tatsache, dass die Berechnung über den Planungshorizont (das Planungszeitfenster) hinausreicht (Abb. 2). Der Berechnungshorizont endet beim Erreichen eines Dg von mindestens $40 \mathrm{~cm}$. Die Bestände, die bereits bei to $40 \mathrm{~cm}$ erreicht haben, werden nicht in dieser Arbeit berücksichtigt, weil für solche Bestände die Endnutzungsphase vorgesehen ist. Wenn ein Bestand zum Zeitpunkt $t_{1}$ (oder früher) einen $\mathrm{Dg} \geq 40$ erreicht, dann stimmt der Berechnungshorizont mit dem Planungshorizont überein $\left(t=t_{1}\right)$. 


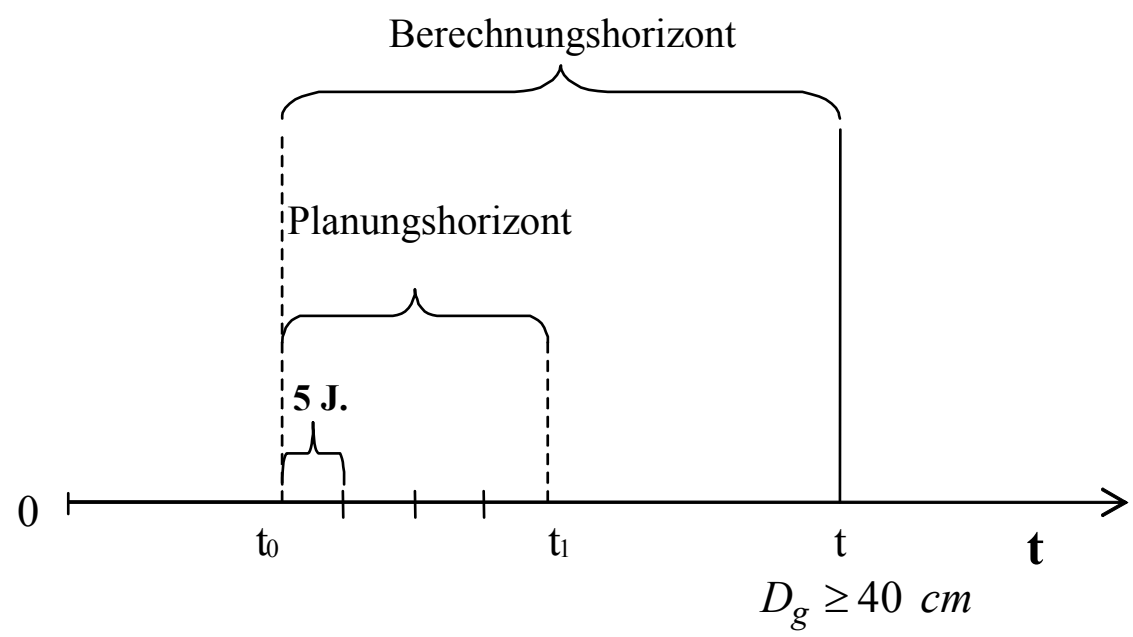

Abb. 2. Schematische Darstellung eines 20-jährigen Planungshorizontes mit vier 5-jährigen Planungsperioden und des Berechnungsfensters zur Schätzung des Bestandeserwartungswertes zum Zeitpunkt t $\left(D_{g} \geq 40\right.$ cm). to: Anfang der Planungsperiode; $t_{1}$ : Ende der Planungsperiode.

Fig. 2. Schematic representation of a 20-year planning horizon with four 5-year planning periods and the computation window for estimation of the forest value at the time $t\left(D_{g} \geq 40 \mathrm{~cm}\right)$. to: Beginning of the planning period; t1: End of the planning period.

Die BEW-Formel (Gl. 9) berechnet den diskontierten Bestandeserwartungswert zum Zeitpunkt $t$, wobei, wie bereits erwähnt wurde, $t$ der Zeitpunkt ist, an dem der Durchmesser des Grundflächenmittelstammes $\left(D_{g}\right)$ gleich oder größer als $40 \mathrm{~cm}$ wird. Zur Berechnung des Terminalwertes und der außerhalb der Planungsperiode gewonnenen Reinerträge (Zeitraum $t_{1}$ bis $t$ ) wurde es für die jüngeren Bestände, die innerhalb des Planungshorizontes einen $\mathrm{D}_{\mathrm{g}}$ von mindestens $40 \mathrm{~cm}$ nicht erreicht haben, nötig, die Berechnungshorizont bis zum Zeitpunkt $t$ $\left(\mathrm{D}_{\mathrm{g}} \geq 40 \mathrm{~cm}\right) \mathrm{zu}$ verlängern. Mit Hilfe von Jakta wurden für die jüngeren Bestände alle möglichen Optionen nicht nur für den 20-jährigen Planungshorizont, sondern bis zum Zeitpunkt $t$ in 5-jährigen Planungsperioden generiert. Der Grund für diese Erweiterung des Berechnungshorizontes ist die Tatsache, dass bei den jüngeren Beständen der Vorrat am Ende des Planungshorizontes noch sehr gering ist. Daher ist der Bestandeswert kleiner als der diskontierte Wert, der bei solchen jüngeren Beständen erreicht werden könnte, wenn sie bis zum Zeitpunkt $t$ bewirtschaftet würden. Abbildung 3 zeigt die unterschiedlichen Entwicklungen des realen Bestandeswertes und des diskontierten Bestandeserwartungswertes. 


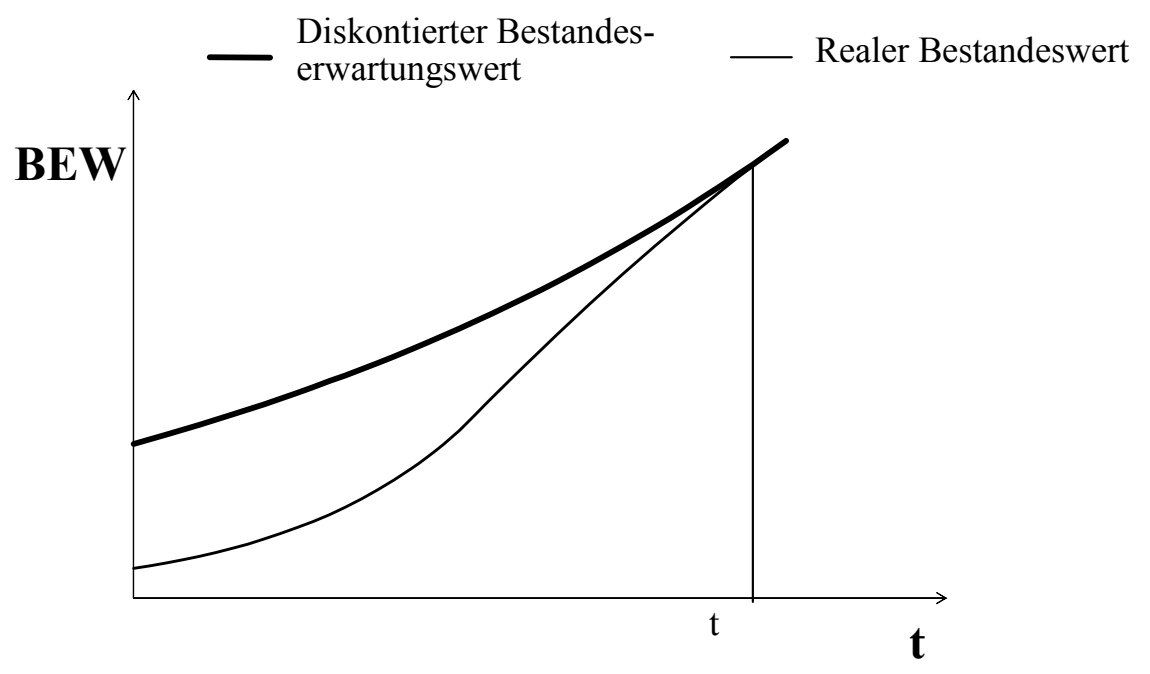

Abb. 3. Unterschied qwischen dem diskontierten Bestandeserwartungswert und dem realen Bestandeswert. $B E W:$ Bestandeserwartungswert.

Fig. 3. Difference between the discounted forest value and the real stand value. BEW: Forest value.

Aus rein ökonomischer Sicht sollte man der Bestand stehen lassen, solange der diskontierte Bestandeserwartungswert den realen Bestandeswert überschreitet. Wenn diese Bedingung nicht erfüllt wird, sollte man den Bestand ernten (Klemperer, 1996; S. 223)

Die BEW-Formel wurde in Jakta Code implementiert. Jakta berechnet automatisch für jede Managementoption den entsprechenden BEW. Für diese Berechnung wurde ein Holzpreis von $80 € \cdot \mathrm{m}^{-3}$ (s. Knoke et al., 2001) und ein Zinssatz von 4\% unterstellt. Eine der Vorteile dieser Formel ist, dass sich die Sensibilität auf Änderungen des zukünftigen Bodenwertes sehr einfach untersuchen lässt, indem man verschiedene $B_{t}$ in der Formel einsetzt. Die Lösung des Optimierungsproblems wird nicht durch den $\mathrm{B}_{\mathrm{t}}$ beeinflusst, weil angenommen wird, dass $B_{t}$ innerhalb des Planungshorizontes konstant ist. Da unser Ziel darin besteht, die Managementoptionen miteinander zu vergleichen, ist es möglich, in der Gleichung (9) auf $\mathrm{B}_{\mathrm{t}} \mathrm{zu}$ verzichten.

\subsection{Die Optimierungsmethode}

Die Entwicklung (und gegebenenfalls Bewertung) von Eingriffsfolgen für einen speziellen Bestand ist Gegenstand der Einzelplanung. Durch die Gesamtplanung kommt es zur Abstimmung der Summe der Einzelplanungen mit der gesamtbetrieblichen Kapazität (Wiegard u. Gadow 1994). Eine Voraussetzung für die erfolgreiche Integration der Einzelund Gesamtplanung ist der Entwurf mehrerer möglicher Eingriffsfolgen für jeden Bestand. 
Daher stellt sich für jeden Bestand die Frage, welche Option hinsichtlich der gesamtbetrieblichen Zielsetzungen und Beschränkungen optimal ist. Für die Lösung des Problems der zeitlich-räumlichen Zuordnung von Managementoptionen bietet sich die Methode der mathematischen Programmierung an (Gadow, 1991).

In dieser Arbeit wurde das Problem der Optimierung der zeitlich- räumlichen Planung nach dem ökonomischen Kriterium des Bestandeserwartungswertes mit Hilfe des Softwarepakets $J L P$ (Lappi, 1992) gelöst. $J L P$ ist ein für Simulationssysteme entwickeltes Softwarepaket zur Lösung von Planungsproblemen. Der Simulator Jakta generiert die Managementoptionen für jeden Bestand und JLP findet die optimale Kombination von Managementoptionen mit Hilfe der Methode der linearen Programmierung. Der Optimierungsalgorithmus verwendet die generalisierte upper bound Methode.

Die lineare Programmierung (LP) ist die am meisten angewandte Technik unter den Methoden der Unternehmensforschung (Operations Research) zur Lösung zahlreicher Entscheidungsprobleme (Rose, 1992). Es ist daher nicht erstaunlich, dass sich viele Anwendungen in der Forstplanung dieser Technik bedienen. Beispiele sind FORPLAN (Johnson et at., 1986) und MELA (Siitonen u. Nuutinen, 1996). Ein Vorteil ist die hohe Berechnungseffizienz der linearen Programmierung. Außerdem lässt sich die Beschränkungsmatrix relativ einfach durch die durch den Simulator generierten Managementoptionen bilden. Die lineare Programmierung kann jeweils nur eine Zielfunktion optimieren und nur unter den eingegebenen Alternativen wählen. Die optimale Lösung ändert sich daher möglicherweise mit der Anzahl und der Art der formulierten Alternativen.

Die wichtigste Annahme der linearen Programmierung bezieht sich auf die Linearität, d. h. die Zielfunktion und die Beschränkungen müssen linear sein (Dykstra, 1984; Nash u. Sofer, 1996). In Bezug auf die Forstplanung bedeutet dies, dass: i) die Nutzung pro Hektar für eine gegebene Managementoption und einen gegebenen Bestand konstant sein muss. ii) Verschiedene Managementoptionen unterschiedlichen Teilflächen eines Bestandes zugewiesen werden können.

Das in dieser Untersuchung verwendete Optimierungsmodell lässt sich wie folgt beschreiben: 
Zielfunktion $\quad$ Max oder Min $z_{0}=\sum_{k=1}^{p} a_{0 k} x_{k}+\sum_{k=1}^{q} b_{0 k} z_{k}$

$$
\begin{aligned}
& c_{t} \leq \sum_{k=1}^{p} a_{t k} x_{k}+\sum_{k=1}^{q} b_{t k} z_{k} \leq C_{t}, \mathrm{t}=1, \ldots, \mathrm{r} \\
& x_{k}-\sum_{i=1}^{m} \sum_{j=1}^{n_{i}} x_{k}^{i j} w_{i j}=0, \mathrm{k}=1, \ldots, \mathrm{p} \\
& \\
& \sum_{j=1}^{n_{i}} w_{i j}=1, \mathrm{i}=1, \ldots, \mathrm{m} \\
& w_{i j} \geq 0 \text { für alle i und j } \\
& z_{k} \geq 0 \text { für } \mathrm{k}=1, \ldots, \mathrm{q}
\end{aligned}
$$

Wobei:

$$
\begin{aligned}
m= & \text { Anzahl der Bestandeseinheiten im Betrieb; } \\
n_{i}= & \text { Anzahl von Managementoptionen für die Bestandeseinheit } i \\
w_{i j}= & \text { Gewicht (Anteil) der Einheit } i \text {, der die Managementoption } j \text { zugewiesen wird; } \\
x_{k}^{i j}= & \text { Menge des ,Produktes“ } k, \text { das von der Bestandeseinheit } i \text { produziert oder } \\
& \text { verbraucht wird, wenn die Managementoption } j \text { angewendet wird; } \\
x_{k}= & \text { erhaltene Menge von Zustandsgrößen } k ; \mathrm{k}=1 \ldots, \mathrm{p}, \\
z_{k}= & \text { zusätzliche Entscheidungsvariablen; } \mathrm{k}=1, \ldots, \mathrm{q}, \\
a_{t k}= & \text { festgelegte reale Konstanten } \mathrm{t}=1, \ldots, \mathrm{r} ; \mathrm{k}=1, \ldots, \mathrm{p}, \\
b_{t k}= & \text { festgelegte reale Konstanten } \mathrm{t}=1, \ldots, \mathrm{r} ; \mathrm{k}=1, \ldots, \mathrm{q}, \\
r & =\text { Anzahl von Beschränkungen. }
\end{aligned}
$$

Der Benutzer von JLP braucht nur die Zielfunktion (Gl. 10) und die Beschränkungen (Gl. 11

- 13) zu definieren. Die restlichen Beschränkungen werden bei der Verwendung der speziellen Problemstruktur automatisch erzeugt.

Für den Waldteil Winnefeld Süd bestand das Ziel darin, die Summe der BEW bis zu dem Zeitpunkt, an dem $\mathrm{D}_{\mathrm{g}} \geq 40 \mathrm{~cm}$ beträgt, zu maximieren, mit der Beschränkung, dass die Reinerträge im Lauf der Planung nicht abnehmen dürfen.

\begin{tabular}{ll} 
Zielfunktion & $\operatorname{Max}_{0}=\sum_{i=1}^{38} B E W_{i t}$ \\
\hline Beschränkungen & $\sum_{i=1}^{38} R E_{i 2}-\sum_{i=1}^{38} R E_{i 1} \geq 0$ \\
\hline & $\sum_{i=1}^{38} R E_{i 3}-\sum_{i=1}^{38} R E_{i 2} \geq 0$ \\
\hline & $\sum_{i=1}^{38} R E_{i 4}-\sum_{i=1}^{38} R E_{i 3} \geq 0$
\end{tabular}


Wobei:

$i=1, . .38 . \quad=$ Anzahl der Bestandeseinheiten im Betrieb;

$B E W$ it $\quad=$ Erwartungswert der $i$-ten Bestandeseinheit zum Zeitpunkt $t$;

$R E_{i k} \quad=$ Reinertrag der Bestandeseinheit $i$ in der $k$-ten Planungsperiode $k=1, . ., 4$.

Die Beschränkung der nicht abnehmenden Reinerträge (Gl. 17-19) impliziert, dass die Summe der Reinerträge aller Bestände in der Simulationsperiode $k$ mindestens gleich der Summe der Reinerträge aller Bestände in der Simulationsperiode k-1 sein muss.

\section{Ergebnisse}

Die Anzahl der mit Jakta generierten Managementoptionen bis zum Zeitpunkt $t$ für den Waldteil Winnefeld Süd beträgt insgesamt 5037. Das in Abbildung 4 gezeigte optimale RaumZeit-Muster entspricht der für das Optimierungsproblem vorgegebenen Zielfunktion und den Beschränkungen, wenn $\mathrm{B}_{\mathrm{t}}=0$ angenommen wird.

\section{Planungsperiode $0-5$ Jahre}

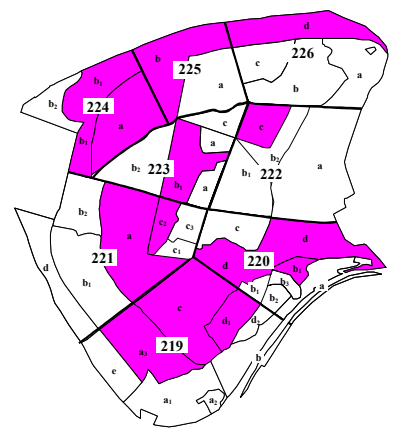

3. Planungsperiode $10-15$ Jahre

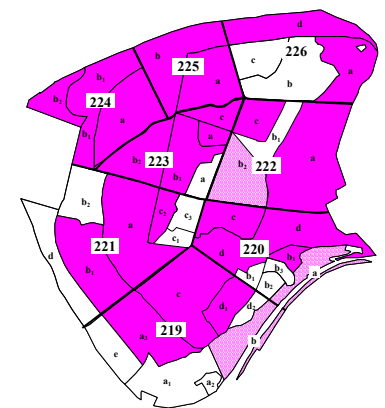

2. Planungsperiode 5 - 10 Jahre

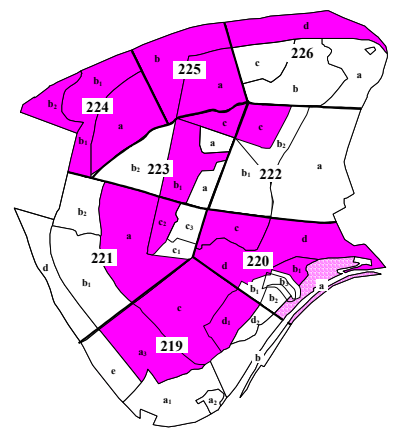

4. Planungsperiode 15 - 20 Jahre

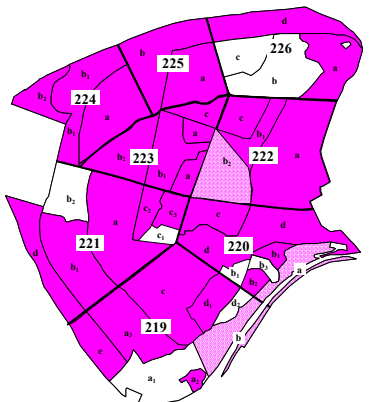

Abb. 4. Zeitlich- und räumliche Planung für den Waldteil Winnefeld Süd. Die dunkel schattierten Flächen werden in der entsprechenden Planungsperiode durchgeforstet, die hell schattierten Flächen befinden sich in der Endnutzungsphase. Holzpreis $=80 € \cdot m^{-3}, \quad r=4 \%, B_{t}=0$.

Fig. 4. Temporal and spatial planning for the forest Winnefeld Süd. The dark shaded areas are going to be thinned in the corresponding planning period, the light shaded areas are in the final harvest phase. Stumpage price: $80 € \cdot m^{-3}, r=4 \%, B_{t}=0$. 
In den dunkel schattierten Flächen sind Durchforstungen vorgesehen. Die hell schattierten Flächen haben den Zieldurchmesser $(40 \mathrm{~cm})$ erreicht und gehen in die Endnutzungsphase über. Für dieses Szenario beträgt der BEW $780981 €^{14}$.

Zur Untersuchung der Auswirkung des Holzpreises auf die forstliche Planung wurde eine Schwankung des Holzpreises von $80 € \cdot \mathrm{m}^{-3} \pm 20 \%$ durchgespielt. Die Abbildungen 5 und 6 zeigen die optimale Lösung für die neuen Holzpreise.

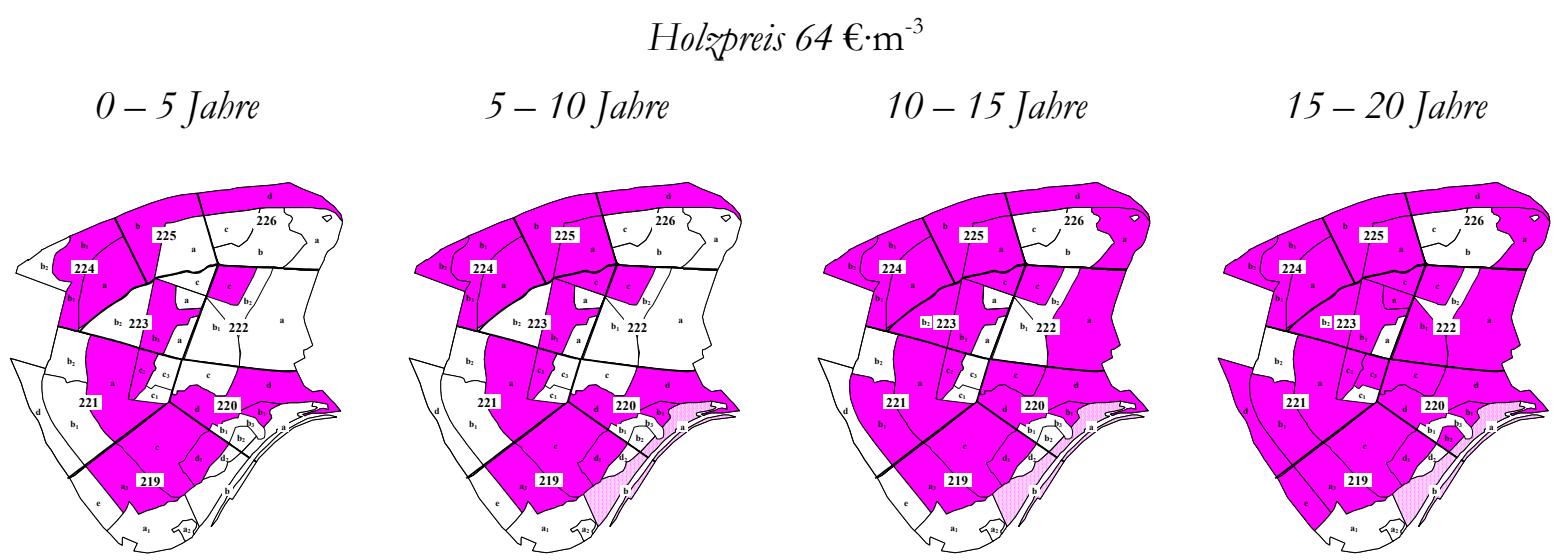

Abb. 5. Optimale Lösung für einen Holapreis von $80 € \cdot m^{-3}-20 \%, r=4 \%, B_{t}=0$.

Fig. 5. Optimum solution for a stumpage price of $80 € \cdot m^{-3}-20 \%, r=4 \%, B_{t}=0$.

$$
0-5 \text { Jahre }
$$

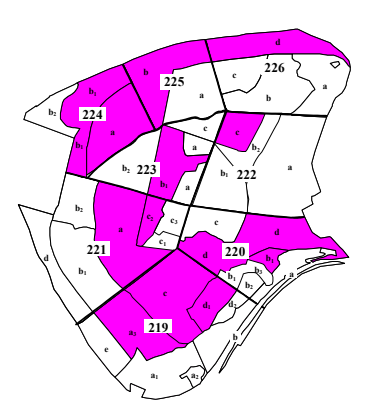

Holipreis $96 € \cdot \mathrm{m}^{-3}$

$$
\text { 5-10 Jabre } \quad 10-15 \text { Jabre }
$$

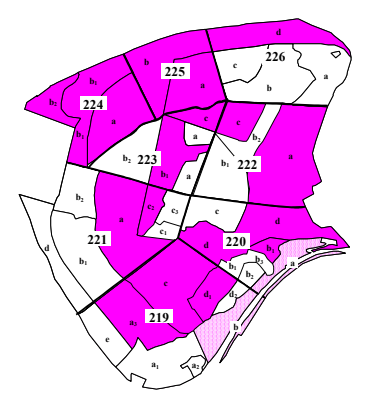

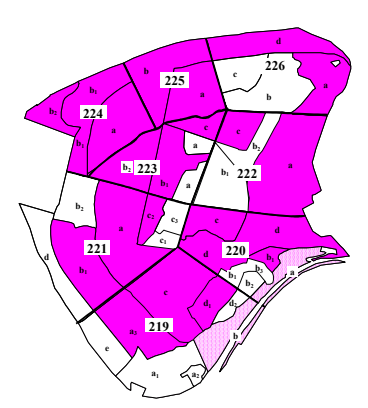

$15-20$ Jahre

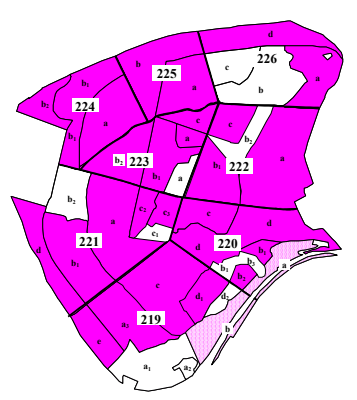

Abb. 6. Optimale Lösung für einen Holzpreis von $80 € \cdot m^{-3}+20 \%, r=4 \%, B_{t}=0$.

Fig. 6. Optimum solution assuming a stumpage price of $80 € \cdot m^{-3}+20 \%, r=4 \%, B_{t}=0$.

\footnotetext{
${ }^{14}$ In der Veröffentlichung ist ein Druckfehler aufgetreten, die in dieser Arbeit korrigiert wurde. Die Angaben über die BEW beziehen sich auf die gesamte Betriebsfläche und nicht auf die Hektar.
} 
Wie aus den Abbildungen 5 und $6 \mathrm{zu}$ entnehmen ist, gibt es kaum Unterschiede in der Reihenfolge der forstlichen Eingriffe; d. h. die Schwankung des Holzpreises hat kaum Einfluss auf das optimale Zeit-Raum-Muster. Dennoch, führt die Schwankung des Holzpreises zu unterschiedlichen Bestandeserwartungswerten; bei einem Holzpreis von 64 $€ \cdot \mathrm{m}^{-3}$ beträgt der BEW $624785 €$. Eine Zunahme des Holzpreises um 20\% hat eine Steigerung des BEW auf $937177 €$ zur Folge.

Ebenfalls wurde der Einfluss einer Veränderung des Zinssatzes auf die optimale Lösung untersucht. Abbildung 7 stellt zwei neue Szenarien für einen Zinssatz von $r=1 \%$ und $\mathrm{r}=7 \%$ dar.

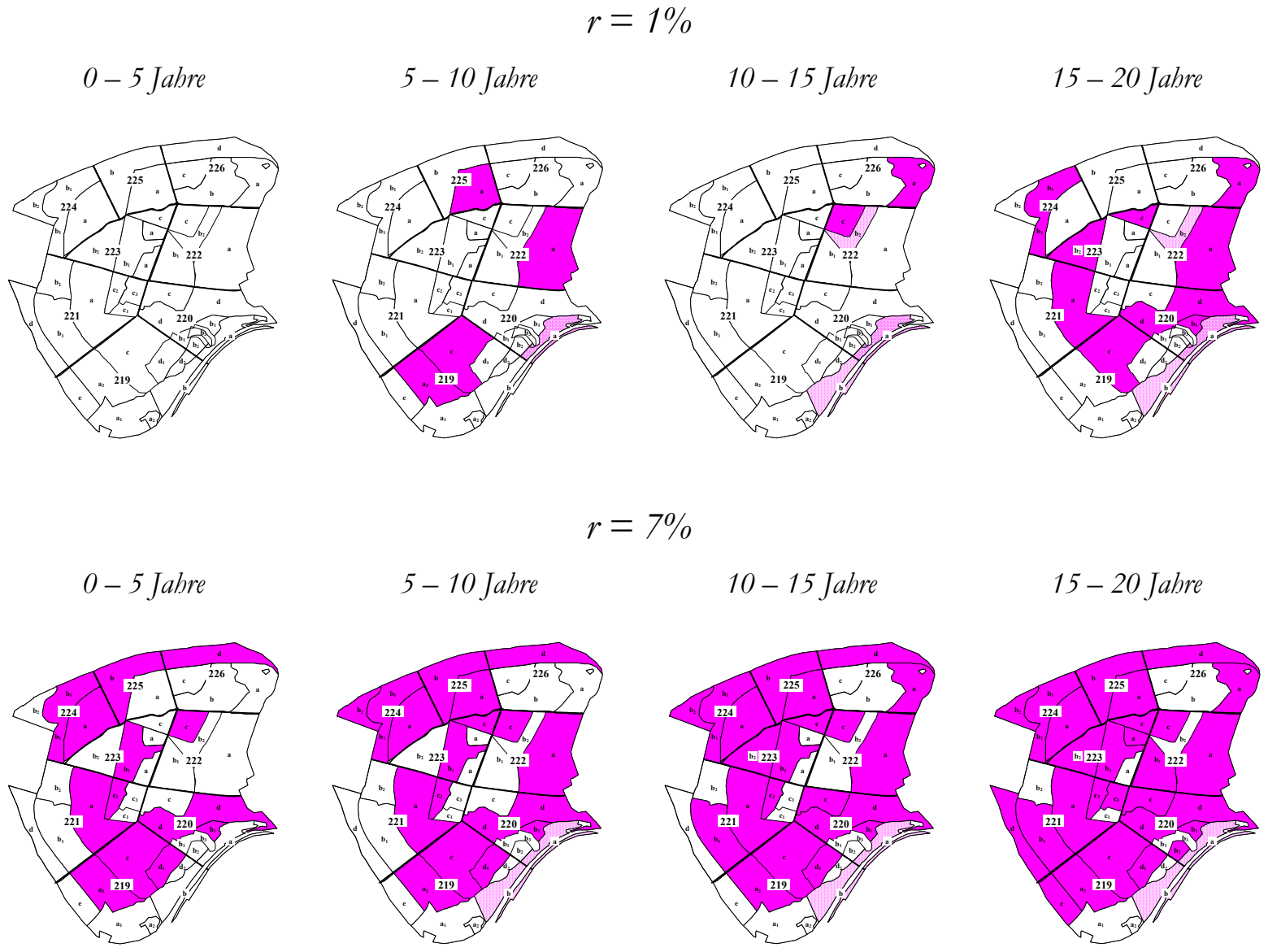

Abb. 7. Optimale Lösungen für verschiedene Zinssätæe: $r=1 \%$ (oben) und $r=7 \%$ (unten), $B_{t}=0$.

Fig. 7. Optimal solution for different interest rates: $r=1 \%$ (up) und $r=7 \%$ (down), $B_{t}=0$.

In der Abbildung 7 ist deutlich zu sehen, dass beim niedrigen Zinssatz kaum forstliche Eingriffe lohnenswert sind. Liegt der Zinssatz hingegen bei 7\%, erzielt eine intensive Forstwirtschaft den größten Erfolg. Beide Szenarien unterscheiden sich nur in dem BEW. 
Für einen Zinssatz von 1\% liegt der BEW bei $2134948 €$, während sich für einen Zinssatz von $7 \%$ ein BEW von $342310 €$ ergibt.

\section{Diskussion}

Die Integration der Einzel- und Gesamtplanung ist ein relevantes Problem mit dem sich die Forsteinrichtung seit Jahrzehnten beschäftigt hat. Die Suche nach dem optimalen Raum-ZeitMuster ist ein kombinatorisches Problem, das schon seit vielen Jahren mit Hilfe des bekannten, von Ware und Clutter (1971) entwickelten „Model I“ gelöst wurde. Wesentliche Beiträge und spezielle Lösungsansätze finden sich u.a. bei Hoganson und Rose (1984), Gadow und Puumalainen (2000) und Öhman (2001).

In dieser Arbeit wurde das Integrationsproblem der Einzel- und Gesamtvornutzungsplanung mit Hilfe des Simulators Jakta zur EDV-gestützten Generierung und Bewertung von Managementoptionen und mit Hilfe des Softwarepakets JLP zur optimalen Lösung der zeitlich-räumlichen Planung gelöst.

Ein Vorteil des Systems Jakta liegt darin begründet, dass es möglich ist, auf der Basis bestimmter Vorgaben eine hohe Anzahl von Managementoptionen für unterschiedliche Ausgangszustände zu generieren. Dieses automatisierte Vorgehen kann auch nachteilig sein. Da die Optionen nach einem festgelegten Muster generiert werden, ist es nicht einfach, die Anzahl der generierten Optionen zu begrenzen und zum Beispiel offensichtlich unpraktische Optionen zu eliminieren.

Mit Hilfe des Systems JLP lässt sich das Optimierungsproblem für das Beispiel Winnefeld mit insgesamt 5037 möglichen Lösungen in wenigen Sekunden lösen. Ein Vorteil der linearen Programmierung gegenüber anderen Optimierungsmethoden, z.B. Simulated Annealing, ist die Tatsache, dass der Rechenaufwand zur Lösung des Problems geringer ist (vgl. Chen u. Gadow, 2002). Außerdem ist es möglich, zahlreiche Beschränkungen zu definieren. Bei Änderung der Zielfunktion oder der Beschränkungen lassen sich sehr einfach neue Entwicklungsszenarien simulieren.

Die graphische Darstellung der optimalen Raum-Zeit-Muster (Abb.4) zeigt deutlich, wann und wo ein Eingriff durchgeführt werden muss. Die Verwendung der Bestandeserwartungswert-Formel (Gl. 9) zur Evaluierung der Managementoptionen hat den Vorteil, dass sich bei Änderung der Marktbedingungen (z.B. Änderung des Holzpreises, Änderung der Zinsrate) die Sensitivität der optimalen Lösung sehr einfach untersuchen lässt. 
Die gesamtbetriebliche Zielsetzung in dieser Arbeit war die Maximierung des Bestandeserwartungswertes. In zukünftigen Arbeiten wäre es auch interessant, Zielfunktionen zu verwenden, die zusätzlich zu den ökonomischen Kriterien weitere Komponenten einbeziehen (z.B. die Förderung der Diversität oder die Minimierung der Schwankungen des periodischen Erntevolumens).

Die Abbildungen 5 und 6 zeigen, dass eine Zunahme oder Senkung des Holzpreises kaum Einfluss auf das optimale Raum-Zeit-Muster hat. Erwartungsgemäß unterscheiden sich die Bestandeserwartungswerte. Je höher der Holzpreis ist, umso höher ist der BEW.

Die Untersuchung der Sensitivität des optimalen Raum-Zeit-Musters auf Schwankungen des Zinssatzes hat gezeigt, dass bei einem kleinen Zinssatz (1\%) nur wenige forstliche Eingriffe durchgeführt werden sollten, während bei einem hohen Zinssatz (7\%) eine intensivere Forstwirtschaft günstig erscheint. Ein Grund dafür ist, dass die Optionen ohne Durchforstung am Ende der Planung einen höheren Vorrat als die Optionen mit Durchforstung haben und deshalb der Terminalwert eines Bestandes ohne Durchforstungen auch höher wird. Unter Berücksichtigung, dass beim kleinen Zinssatz der Terminalwert nur leicht diskontiert wird, sind die Bestandeserwartungswerte der Managementoptionen ohne Durchforstungen höher als die BEW der Managementoptionen mit Durchforstung, in welchen die Reinerträge der Durchforstungen nicht genügen, um den BEW zu erhöhen. Bei einem höheren Zinssatz (7\%) wird der Terminalwert stark diskontiert, deswegen sind die $\mathrm{BEW}$, sogar für die Managementoptionen mit Durchforstung gering. Die Ergebnisse der Untersuchung entsprechen der ökonomischen Theorie: je höher der Zinssatz ist, umso weniger rentabel ist die Investition (s. Williams, 1981; S. 17). Frühere Untersuchungen bestätigten, dass ein hoher Zinssatz das Bewirtschaftungssystem der Dauerwaldnutzung mit häufigen und regelmäßigen Eingriffen begünstigt (Chang, 1981).

\section{Danksagung}

Wir bedanken uns sehr herzlich bei Herrn Dr. Juha Lappi für die Unterstützung beim Programmieren und für zahlreiche Hinweise. Für die methodische Unterstützung bei der Bewertung von Optionen danken wir Herrn Prof. Dr. Chang. Zu danken haben wir Herrn Prof. Dr. von Gadow für die wissenschaftliche Anleitung und zahlreiche methodische Hinweise, sowie Dr. Matthias Albert für die kritische Durchsicht dieses Manuskriptes. Wir danken auch den Mitarbeitern im Institut für Waldinventur und Waldwachstum, 
insbesondere den Teilnehmern an unserem Doktorandenseminar für die zahlreichen Diskussionen zu diesem Thema.

\section{Summary}

The aim of this analysis is to integrate the planning of the thinning phase at stand level and at forest level, based on an example 38 spruce stands of the forest Winnefeld Süd. The solution of this problem requires the generation, evaluation and finally the optimization of management options. For the generation of the management options a growth model and a thinning model were used. With the management simulator Jakta several options for each stand were generated. For the evaluation of the options the economic criterion of forest value (BEW) was applied. The optimization is concerned with the evaluation of multiple scenarios for the whole forest, involving the determination of the optimal space-time pattern of thinnings for all the stands combined. For this purpose the software package $J L P$ was used. Finally a sensitivity analysis is carried out for the optimal solution, considering changes in timber price and interest rate.

Keywords: Forest planning, optimization, generating management options, forest value, evaluation, spruce stands.

\section{Literatur}

Chang, J. S., 1981: Determination of the optimal growing stock and cutting cycle for an uneven-aged stand. For. Sci. 27(4):739-744.

Chen, B. W. und Gadow, K. v., 2002: Timber harvest planning with spatial objectives, using the method of simulated annealing. Forstw. Cbl. 121(1):25 - 34 .

Döbbeler, H. und Spellmann, H., 2002: Methological approach to simulate and evaluate silvicultural treatments under climate change. Forstw. Cbl. 121, Supplement 1:52-69.

Dykstra, D., 1984: Mathematical programming for natural resource management. McGrawHill. New York, $318 \mathrm{~S}$.

Gadow, K. v., 1991: Integration von Einzel- und Gesamtnutzungsplanung in der Forsteinrichtung. Allg. Forst- u. J. Ztg. 162 (4):72-75.

Gadow, K. v. und Puumalainen, J., 2000: Scenario planning for sustainable forest management. In: Gadow, K. v., Pukkala, T. and Tomé, M., 2000: Sustainable Forest Management. Kluwer Series Managing Forest Ecosystems, Vol 1: 319-356.

Gadow. K. v., 2001: Forsteinrichtung - Alternativen zur Kontrollstichprobe. Forsteinrichtung für das 21. Jahrhundert. Tagungsbericht zum Kolloquium des Ladesforstvereins Sachsen-Anhalt. 7. Februar 2001 in Drei Annen-Hohne. SachsenAnhalt Landesforstverwaltung. S. 110-120. 
Gadow, K. v. und Hui. G. Y., 1999: Modelling forest development. Kluwer Academic Publishers, Dordrecht, $213 \mathrm{~S}$.

Gurjanov, M., Sánchez Orois, S. und Schröder, J., 2000: Grundflächemodelle für gleichaltrige Fichtenreinbestände. Eine vergleichende Analyse. Centralblatt für das gesamte Forstwesen 117(3/4):187-198.

Hille, M., Hessenmöller, D., Möhring, B. und Gadow, K. v., 1999: Bewertung waldbaulicher Maßnahmen mit Hilfe der Zeitfenstermethode am Beispiel eines 110jährigen Buchenbestandes. IUFRO Proceedings, Prague.

Hoganson, H. M. und Rose, D. W., 1984: A simulation approach for optimal timber management scheduling. For. Sci. 30(1):220 - 238.

Johnson, K. N., Stuart. T. W. und Crim, S. A., 1986: FORPLAN Version 2: An overwiew. USDA Forest Service Land Management Planning System Section. Washington DC.

Kassier, H. W., 1993: Dynamics of diameter and height distributions in commercial timber plantations, PhD dissertation, Faculty of Forestry, Univ. of Stellenbosch, South Africa.

Klemperer, W. D., 1996: Forest resource economics and finance. McGraw-Hill, Inc.

Knoke, T., Moog, M. und Plusczyk, N., 2001: On the effect of volatile stumpage prices on the economic attractiveness of a silvicultural transformation strategy. Forest Policy and Economics 2(3-4):229 - 240.

Kramer, H., 1990: Nutzungsplanung in der Forsteinrichtung. 2. Auflage. J. D. Sauerländer's Verlag. Frankfurt/Main.

Lappi, J., 1992: JLP - A linear programming package for management planning. The Finnish Forest Research Institute. Research papers 414 Suonenjoki, 134 S.

Lappi, J., 2002: Jakta user's guide (unveröffentlichtes Manuskript).

Nash, S. G. und Sofer. A., 1996: Linear and non linear programming. The Mcgraw-Hill Companies. Inc., $692 \mathrm{~S}$.

Öhman, K., 2001: Forest planning with consideration to spatial relationships. Doctoral Thesis. Swedish University of Agricultural Sciences, Umeå.

Rodríguez Soalleiro. R., 1995: Crecimiento y producción de masas forestales regulares de Pinus pinaster Ait. en Galicia. Alternativas selvícolas posibles. Ph D. Dissertation. Escuela Técnica Superior de Ingenieros de Montes. Madrid, 297 S.

Rose, D., 1992: Quantitative Modelle in der strategischen Planung am Beispiel der Forstwirtschaft, Hochschul Verlag.

Sánchez Orois, S., Gurjanov, M. und Schröder, J., 2001: Analyse des Grundflächenzuwachses gleichaltriger Fichtenreinbestände. Allg. Forst- u. J. Ztg. 172(3):51-60.

Schübeler, D., 1997: Untersuchungen zur standortabhängigen Wachstumsmodellierung bei der Fichte. Dissertation zur Erlangung des Doktorgrades der Forstlichen Fakultät der Georg-August Universität in Göttingen, 155 S.

Siitonen, M. und Nuutinen. T., 1996: Timber production analyses in Finland and the MELA system large-scale forestry scenario models: experiences and requirements. Joensuu. Finland. 15-22 June 1995. EFI proceedings. no. 5.

Spellmann, H., Nagel, J. und Böckmann, T., 1999: Summarische Nutzungsplanung auf der Basis von Betriebsinventurdaten. Allg. Forst- u. J. Ztg. 170(7):122-128.

Staupendahl, K., 1999: Bestandesbezogene Modellierung von Durchforstungen in Buchenreinbeständen auf der Basis des Stammzahl-Grundflächen-Verhältnisses. Vortrag anlässlich der Jahrestagung der Sektion Ertragskunde des Deutschen Verbandes Forstlicher Forschungsanstalten in Volprehausen/Solling am 17. - 19. 5. 1999. 
Staupendahl, K. und Puumalainen, J., 1999: Modellierung des Einflusses von Durchforstungen auf die Durchmesserverteilung von gleichaltrigen Fichtenreinbeständen. Centralblatt für das gesamte Forstwesen 116(4):249-262.

Vilčko, F., 2002: Zur Quantifizierung forstlicher Eingriffe und Ertragsprognose in Fichtenbestände. Institut für Forsteinrichtung und Ertragskunde, Georg-AugustUniversität Göttingen. (Unveröffentlichtes Manuskript).

Vilčko, F. und Gadow, K. v., 2002: Konstruktion eines alterabhängigen Bestandesmodells für Fichte (Picea Abies L. Karst). Institut für Forsteinrichtung und Ertragskunde, Georg-August-Universität Göttingen. (Unveröffentlichtes Manuskript).

Ware, G. O. und Clutter, J. L., 1971: A mathematical programming system for the management of industrial forests. For. Sci. 17:428 - 445.

Wiegard, C. und Gadow, K. v., 1994: Beispielhafte Darstellung eines integrierten Planungsmodells für einen Buchen-Edellaubholz-Mischwald. Unveröffentlichtes Manuskript; Institut Forsteinrichtung und Ertragskunde, Georg-August-Universität Göttingen.

Wikström, P., 2000: Solving stand-level planning problems that involve multiple criteria and a single-tree growth model. Doctoral Thesis. Swedish University of Agricultural Sciences, Umeå. 



\section{Veröffentlichung IV}

Modelling the growth and management of mixed uneven-aged maritime pine-broadleaved species forest in Galicia NW Spain

SÁNCHEZ OROIS, S. UND RODRÍGUEZ SOALLEIRO, R.

Erschienen in:

Scandinavian Journal of Forest Research. Jahrgang 2002, 17(6): 537-547. 



\title{
Modelling the growth and management of mixed uneven-aged maritime pine-broadleaved species forests in Galicia (NW Spain)
}

\author{
SOFÍA SÁNCHEZ OROIS AND ROQUE RODRÍGUEZ SOALLEIRO \\ Institut für Forsteinrichtung und Ertragskunde. Georg-August-Universität Göttingen. \\ Büsgenweg 5, 37077 Göttingen, Germany
}

\begin{abstract}
The state of mixed forests of maritime pine (Pinus pinaster Ait.) and broadleaved species in the coastal region of Galicia (Northwestern Spain) was described using data from 213 circular sample plots selected among the available 4700 plots of the Spanish National Inventory. A matrix model was developed for this forest type to obtain information about the productivity and potential for sustainable management. The broadleaved species had a diameter distribution close to the inverted $j$-shape typical of the uneven-aged forests but for maritime pine there were many medium size trees and a lack of trees in the first DBH class of $15 \mathrm{~cm}$. The matrix growth model was used to predict the development of mixed forests for three different management options: no harvesting in a well stocked stand, a regime with 5-year harvest cycle and a residual basal area of $15 \mathrm{~m}^{2} \mathrm{ha}^{-1}$ in a well stocked stand, and a rehabilitation management applied to an understocked stand. The results showed the possibility to apply uneven-aged silvicultural systems to these forests leading to the production of high valuable timber and to the maintenance of a continuous cover and biodiverse forest.
\end{abstract}

Keywords: Matrix-growth model, maritime pine, mixed stands, uneven-aged, simulation. 


\section{Introduction}

Maritime pine (Pinus pinaster Ait.) is one of the most important commercial forest species in Galicia (northwestern Spain) where it covers about the $47 \%$ of the forest area (620000 ha) in pure or in mixed stands. The total timber volume of this species amounts to 50 million $\mathrm{m}^{3}$ and the annual harvest level is about 2,5 million $\mathrm{m}^{3}$. Accordingly, $P$. pinaster is the most important commercial species in northwestern Spain (DGCONA, 2000).

The silviculture of this species in Galicia reached importance three centuries ago when agricultural landowners started to sow pine nuts in their intermittently worked rye lands. The grain was harvested and the pine seedlings were left to grow for very short rotations (Ruíz Zorrilla, 1981). This type of culture and the special ability of maritime pine to regenerate naturally, especially after burning, lead to a rapid expansion in the coastal areas and a naturalization of the pine. Another important factor promoting the expansion of the species were the reforestation programs developed by the Forest Administration on Communal Lands from 1940 to 1970 , especially in the inland areas.

The current irregular structure of the forests on private land is a result of the management, which is very different from that applied by the Forest Service. The private forests are nonindustrial properties of small size, less than 1 ha on average. The management normally involved a kind of high grading of the stands, i.e. only the best quality and the largest trees were harvested when cash income was required by the owners, leaving trees of poor growth and low quality (Molina, 1988). The result is an uneven-aged forest, understocked in many cases and lacking commercially valuable trees (Romero, 1992).

A special type of pine forest with a mixture of broadleaved species and pines of different ages has developed as a result of this practice, especially on high productivity sites. The total area of these forests amounts to 87000 ha in the coastal region of Galicia. The traditional forest administration view was to consider this type of forest "irrational", suggesting that the productivity is only a third of the pure even-aged stands (Xunta de Galicia, 1992).

However, growth and yield data for this forest type were not available until recently. The possibility of active management of these lands, applying uneven-aged silviculture, remains unexplored. Different types of silvicultural treatments designed to transform stand structures to the even-aged system have been developed in northern Portugal for private forests with similar origin and structure (Loureiro, 1991). Under certain conditions selection 
management might be financially superior to even-aged management for landowners (Redmond and Greenhalgh, 1990). Chang (1990) found that higher interest rates favored uneven-aged management, since infrequent large revenues from even-aged stands lead to lower net present values at high interest rates than the smaller but more frequent revenues from uneven-aged stands. Indeed, one benefit of selection silviculture is its ability to produce high sawtimber yields (Schulte and Buongiorno, 1998).

The purpose of this study was to describe the present average condition of the unevenaged forest type according to data from the second and third Spanish National Survey, to develop a matrix growth model for this forest type and finally to simulate and analyse the development of well stocked and understocked stands using different management regimes with the proposed matrix growth model.

\section{Materials and methods}

\subsection{Tree data}

The data were derived from 213 circular sample plots selected among the available 4700 plots of the Spanish National Inventory which are located in the provinces of $A$ Coruña and Pontevedra, in the autonomous region of Galicia (Fig. 1).

\section{Atlantic \\ Ocean}

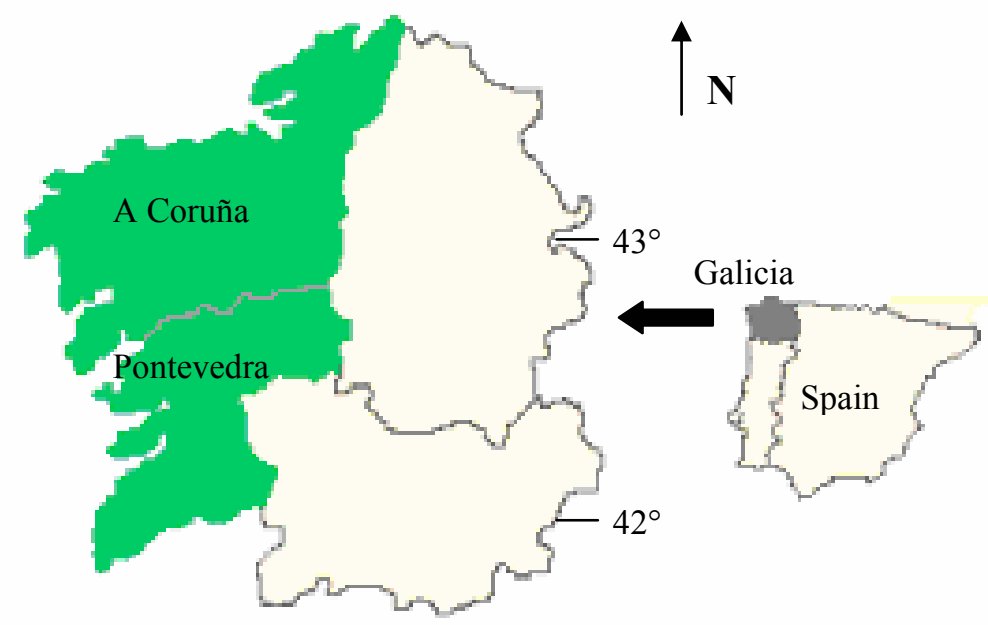

Fig. 1. Location of the provinces A Coruña and Pontevedra in north-western Spain. 
Galicia has a mild and humid climate with a high timber production potential. The average annual rainfall ranges from 1000 to $2500 \mathrm{~mm}$, and in contrast to the Mediterranean climate, there are no dry summers. The average annual temperature is higher than $12{ }^{\circ} \mathrm{C}$ in the coastal provinces of A Coruña and Lugo. The soils are acid, with poor fertility and the parent materials are mainly granites and schists or slates. Maritime pine, or the Atlantic form of $P$. pinaster, occurs naturally in SW France, NW Spain and North Portugal. It is a medium-sized tree which can reach heights of 20-30 $\mathrm{m}$ and endures acid, poor and sandy soils. Pure and mixed stands (P. pinaster mixed with Eucalyptus globulus Labill.) of maritime pine dominate the forest composition in Galicia followed by pure stands of eucalypts and mixed stands of pines-broadleaved species, mainly Quercus robur L. (DGCONA, 2000).

For this study 213 plots of the National Forest Inventory were selected using the following criteria:

a) the plots should have a mixture of broadleaved species and maritime pine with at least $10 \%$ of stocking in each of the two groups,

b) eucalypts should not be present within the plot,

c) the plots should have a large diameter range, with at least 5 diameter classes being represented.

All trees in the plot were numbered and identified by their azimuth-angle and distance to the plot centre. Each plot was measured twice, first in 1987 and again in 1997. The plots were of concentric circular design with varying threshold diameters: all trees between 12.5 and 22.5 $\mathrm{cm}$ diameter at breast height ( $\mathrm{dbh}$ ) were measured in the inner circle of $10 \mathrm{~m}$ radius; all trees between 22.5 and $42.5 \mathrm{~cm}$ were measured in the next bigger circle of $15 \mathrm{~m}$ radius; trees exceeding $42.5 \mathrm{~cm}$ dbh were measured in the last circle with a radius of $25 \mathrm{~m}$.

In the analysis a diameter class width of $5 \mathrm{~cm}$ was used, the lower bound class midpoint being $15 \mathrm{~cm}$ and the upper one being $60 \mathrm{~cm}$. Weighting factors were used to calculate the number of trees and basal areas per ha for each diameter class. The stand structure expressed as the number of trees in each diameter class in each species, was then determined for both inventories. The trees harvested between the two surveys were assumed to have been cut in the middle of the interval (in year 5). Tree volumes were calculated using the equations proposed by the Spanish National Survey (ICONA, 1993). 


\subsection{Matrix growth model}

A matrix stand growth model was developed from the data. The following model in which the growth parameters are functions of the stand density, expressed by basal area, was used to simulate stand development in accordance with the approach proposed by Buongiorno et al. (1995):

$$
y_{t+1}=G\left(B_{t}\right)\left(y_{t}-h_{t}\right)
$$

Where:

$\mathbf{y}_{\mathbf{t}}=\left[\mathrm{y}_{\mathrm{itt}}\right]-$ Column vector representing the state of the stand at time $\mathrm{t} ; \mathrm{i}=1 \ldots \mathrm{n}$, indicates the tree species $; j=1 . . m$ the diameter size class.

$\mathbf{h}_{\mathbf{t}}=\left[\mathrm{h}_{\mathrm{ijt}}\right]-$ Column vector of the number of trees of species $\mathrm{i}$ and diameter class $\mathrm{j}$ harvested at time $\mathrm{t}$.

$\mathbf{G}=$ Growth matrix as function of the basal area $\mathbf{B}$. Its parameters represent the effects of stand density and number of trees on ingrowth, as well as the probabilities that a tree stays in a diameter class or moves into a larger class during the interval t to $\mathrm{t}+1$.

The ingrowth values of the 10 -year period (the number of trees per ha entering in the first diameter class) were calculated from the data of the second inventory, assuming a constant annual rate of ingrowth. Both in conifers and broadleaved trees, plots with positive ingrowth were separated from those with zero ingrowth. The aim was to fit a logistic model to determine the ingrowth probability (Hamilton, 1986; Woollons, 1998). A predictive equation was then fitted for all the plots with ingrowth, using a logarithmic transformation, thus ensuring normality. The application of the model requires generating a random number, which is compared with the probability level associated with a positive ingrowth (Fig. 2). The following logistic model was used:

$p_{i}=\frac{1}{1+e^{b_{0}+b_{1} x_{1}+\ldots .+b_{n} x_{n}}}$

Where:

$$
\begin{aligned}
& \mathrm{p}_{\mathrm{i}}=\text { probability of ingrowth; } \\
& \mathrm{x}_{\mathrm{i}}=\text { independent variables used in the model; } \\
& \mathrm{b}_{\mathrm{i}}=\text { coefficients. }
\end{aligned}
$$




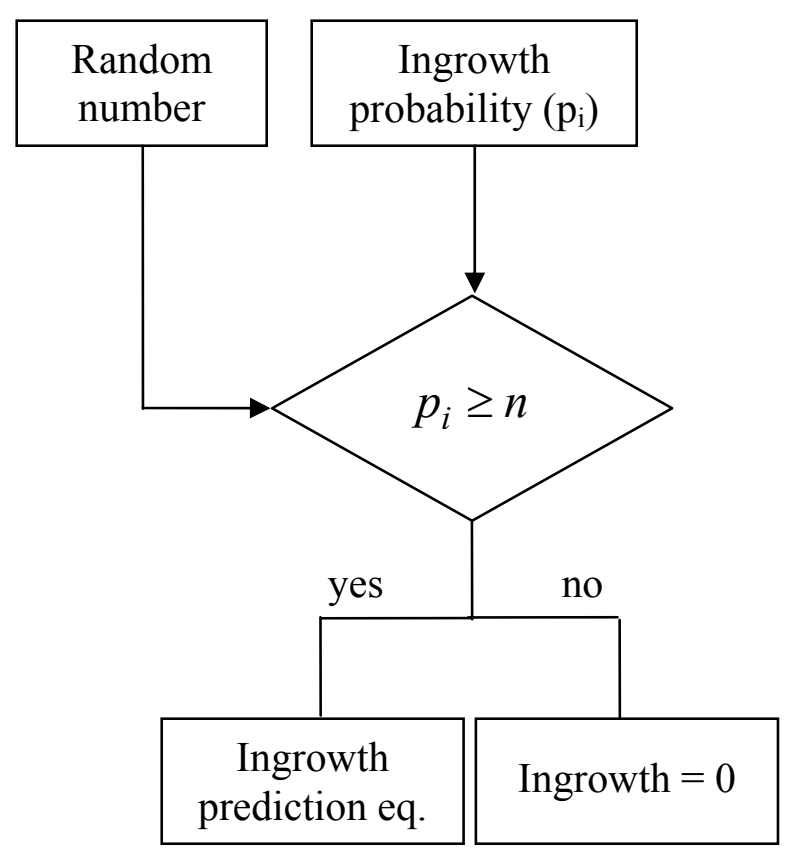

Fig. 2. Schematic representation of the use of the logistic model.

The model may be applied annually, assuming that both ingrowth and mortality are continuous processes and allowing the calculation of annual values from 10 -yearly estimates. The SAS Stepwise procedure was used in all cases to select the variables entering in the logistic regression model with a combination of stand variables, especially number of stems and basal area per hectare. The upgrowth probabilities (the probability of a tree growing up from one diameter class to the next) were calculated for a one-year period using the diameter growth divided by the diameter class width and this result divided by ten, to avoid values greater than 1 (Gadow and Hui, 1999). For the mortality model a logistic binary approach [0, 1] was applied representing the mortality in 10 years.

The growth model recognizes 10 diameter classes and two species groups: maritime pine and broadleaved trees (which include mainly Quercus robur L., Castanea sativa Mill. and occasionally Quercus suber L., Quercus pyrenaica Wild., Betula celtiberica Rothm and Vasc., Alnus glutinosa L. or Fraxinus sp). The regressions were calculated using the procedure REG of the statistical software SAS (SAS 1988). 


\subsection{Simulation}

The matrix model was used to predict the development of mixed forests during a period of 20 years, starting in year 1997, using two hypothetical initial states and three different management treatments:

1. a well-stocked stand

a) no harvesting

b) a specific sustainable harvest regime

2. an understocked stand

a) a rehabilitation regime

The initial composition of a well stocked stand was considered to be equal to the average composition of the last survey. For the understocked stands, an initial state was constructed, with a third of the average basal area, giving a value less than the reference value of $11 \mathrm{~m}^{2} \mathrm{ha}^{-1}$ used in the mixed stands of loblolly pine (Pinus tadea L.) in the south-eastern USA (Baker, 1989).

In the well stocked stand a harvesting cycle of 5 years, a residual basal area of the harvested stands of 16 to $17 \mathrm{~m}^{2} \mathrm{ha}^{-1}$ and a maximum diameter of $60 \mathrm{~cm}$ of the harvested trees were applied according to the experience on selection management for pine or oak-pine stands (Schulte and Buongiorno, 1998). The criteria for removal of trees were as follows:

Broadleaved species - A third of the trees were removed in each diameter class but the highest, where $80 \%$ of the trees were removed.

Pines - No harvest in the first diameter class, from the second to the penultimate diameter class 60 per cent of the difference between the number of trees in 2 subsequent classes $(i, i+1)$ were removed in the smaller class $i$.

In the understocked stand starting with an initial basal area of $7.3 \mathrm{~m}^{2} \mathrm{ha}^{-1}$, harvesting was concentrated on the understory hardwoods $40 \%$ of the trees in the first dbh-class were removed) with the aim of holding back this species group. In the other diameter classes, 15\% of the trees were removed every 5 years, $40 \%$ of stems were removed in the highest dbhclass. The harvest level in pines was $50 \%$ of the harvest regime for a well stocked stand. 


\section{Results}

\subsection{Current state}

In the first inventory, the average stand had a basal area of $18.9 \mathrm{~m}^{2} \mathrm{ha}^{-1}$ and 329.8 stems ha-1, of which $82 \%$ were pines and $14 \%$ broadleaved species (Table 1). After a harvest of $6.1 \mathrm{~m}^{2}$ ha-1 or $45.2 \mathrm{~m}^{3} \mathrm{ha}^{-1}$ in ten years, the stands had an average before-harvest basal area in the second inventory of $21.8 \mathrm{~m}^{2} \mathrm{ha}^{-1}$ and a stocking of 362.7 trees ha- $\mathrm{h}^{-1}$ of which $67 \%$ are pines and $33 \%$ broadleaved species.

\section{$1^{\text {st }}$ inventory (1987) Harvested $\quad 2^{\text {nd }}$ inventory (1997)}

\begin{tabular}{ccccccc}
\hline Dbh class & Pines & Broadleaved & Pines & Broadleaved & Pines & Broadleaved \\
\hline 15 & 68.9 & 19.0 & 32.4 & 3.6 & 55.3 & 44.7 \\
20 & 56.2 & 9.3 & 18.7 & 0.9 & 40.2 & 20.0 \\
25 & 48.8 & 8.6 & 17.9 & 1.9 & 34.5 & 11.0 \\
30 & 42.9 & 6.5 & 15.4 & 0.7 & 33.7 & 8.4 \\
35 & 26.7 & 6.2 & 8.6 & 0.5 & 27.3 & 6.4 \\
40 & 16.1 & 3.4 & 6.1 & 0.5 & 22.9 & 5.0 \\
45 & 6.0 & 2.0 & 2.4 & 0.2 & 10.2 & 2.6 \\
50 & 3.5 & 1.2 & 1.1 & 0.1 & 6.6 & 2.0 \\
55 & 1.8 & 0.7 & 0.7 & 0.0 & 3.8 & 1.0 \\
$60+$ & 1.3 & 0.6 & 0.6 & 0.0 & 3.6 & 1.2 \\
\hline Total & 272.2 & 57.6 & 103.9 & 8.5 & 238.1 & 102.3 \\
\hline
\end{tabular}

Table 1. Average diameter distribution before harvest in the $1^{\text {st }}$ inventory, harvested trees between inventories and diameter distribution before harvest in the $2^{\text {nd }}$ inventory.

The average harvest rate of $4.52 \mathrm{~m}^{3} \mathrm{ha}^{-1} \mathrm{yr}^{-1}$ during the last ten years seems to be low enough to produce increments of $19 \%$ in the average timber volume and $15.3 \%$ in the average basal area, thus leading to better stocked stands that also have a higher broadleaved species proportion. This was especially obvious when analyzing the first $15 \mathrm{~cm}$ diameter class where the average number of broadleaved species increased from 19 trees ha-1 in the first inventory to 44.7 trees ha-1 in the second, and that of pines fell from 68.9 to 55.3 trees ha-1.

In the second inventory, the broadleaved species diameter distribution shows a socalled inverted $j$ shape with a calculated average q value equal to 1.56 . The maritime pine distribution showed a loss of trees in the lower DBH classes.

In the broadleaved group, the most important species was oak, representing $65 \%$ of the broadleaved tree-stems in Coruña and $74 \%$ in Pontevedra. Chestnut had quite a different representation in both provinces, with $27 \%$ in Coruña and $12 \%$ in Pontevedra. 
The harvest had been concentrated on the valuable pine products, with $34 \%$ of the total harvested timber coming from pines of more than $35 \mathrm{~cm} \mathrm{DBH}$. A calculation of the percentage of timber grades specified as a function of the thin-end diameter of the log gave the following results: Grade I $(>35 \mathrm{~cm}), 15.4 \%$ of the pine timber, grade II $(22-35 \mathrm{~cm})$, $34 \%$, grade III $(<22 \mathrm{~cm}), 50.6 \%$. Broadleaved species corresponded to only $5 \%$ of the total timber harvested.

\subsection{The matrix growth model}

Equations 3 to 6 show the results of fitting the data to the logistic model for calculating the probability of ingrowth and mortality. Tables 2 to 3 show the results of fitting the growth matrix to the data. In all cases the parameter values were calculated for both the pine and broadleaved species.

The ingrowth probability for a 10 -year period is estimated as follows:

$p_{c}=\frac{1}{1+e^{-0.2409-0.0115 \cdot N_{c}+0.1608 \cdot G_{c}}}$ for conifers

$p_{b}=\frac{1}{1+e^{-1.3273+0.0522 \cdot G}}$ for broadleaved species

Where:

$\mathrm{p}_{\mathrm{c}}=$ Ingrowth probability for conifers;

$\mathrm{p}_{\mathrm{b}}=$ Ingrowth probability for broadleaved species;

$\mathrm{N}_{\mathrm{c}}=$ Stems per ha (conifers);

$\mathrm{G}_{\mathrm{c}}=$ Basal area $\mathrm{m}^{2} \mathrm{ha}^{-1}$ (conifers);

$\mathrm{G}=$ Total basal area $\mathrm{m}^{2} \mathrm{ha}^{-1}$.

The estimating function for the mortality probability in a 10 -year period is:

$m_{c}=\frac{1}{1+e^{4.3938-0.0621 \cdot G_{c}+0.000937 \cdot G_{c}^{2}-0.00410 \cdot D+0.000011 \cdot D^{2}}}$ for conifers

$m_{b}=\frac{1}{1+e^{3.9097-0.00266 \cdot D}}$ for broadleaved species

Where:

$\mathrm{m}_{\mathrm{c}}=$ Mortality probability for conifers;

$\mathrm{m}_{\mathrm{b}}=$ Mortality probability for broadleaved species;

$\mathrm{G}_{\mathrm{c}}=$ Basal area $\mathrm{m}^{2} \mathrm{ha}^{-1}$ (conifers);

$\mathrm{D}=\operatorname{Diameter}(\mathrm{cm})$. 
Equations of ingrowth for broadleaved species: $I_{b}=\alpha+\beta \cdot N_{b}+\gamma \cdot B \cdot N_{b}$

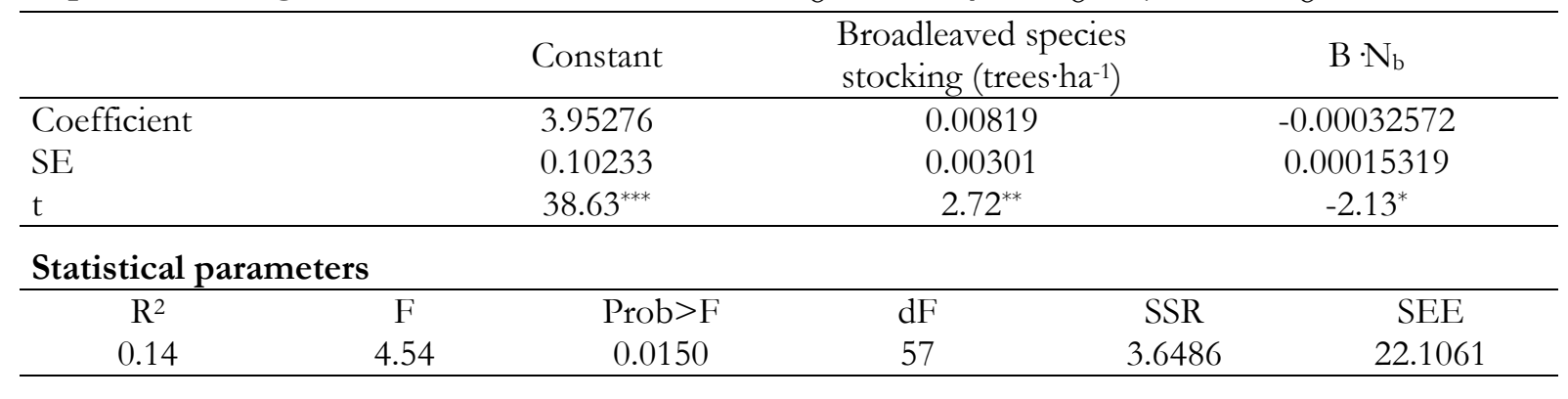

\begin{tabular}{|c|c|c|c|c|}
\hline \multicolumn{5}{|c|}{ Equations of ingrowth for pines: $I_{c}=\alpha+\beta \cdot N_{c}+\delta \cdot N_{b}+\gamma \cdot B \cdot N_{c}$} \\
\hline & Constant & $\begin{array}{c}\text { Pines stocking } \\
\left(\text { trees }^{\prime} \cdot \mathrm{ha}^{-1}\right)\end{array}$ & $\begin{array}{c}\text { Broad. stocking } \\
\left(\text { trees } \cdot \mathrm{ha}^{-1}\right)\end{array}$ & $\mathrm{B} \cdot \mathrm{N}_{\mathrm{c}}$ \\
\hline Coefficient & 4.12018 & 0.00231 & -0.00255 & $-0.8559 \cdot 10^{-4}$ \\
\hline SE & 0.15858 & 0.00096 & 0.00097 & $0.3042 \cdot 10^{-4}$ \\
\hline t & $25.98^{* * *}$ & $2.40^{*}$ & $-2.62^{*}$ & $-2.81^{* *}$ \\
\hline \multicolumn{5}{|c|}{ Statistical parameters } \\
\hline $\mathrm{R}^{2}$ & $\mathrm{~F}$ & Prob $>F$ & SSR & SEE \\
\hline 0.16 & 6.93 & 0.0003 & 9.3724 & 48.6854 \\
\hline
\end{tabular}

Table 2. 10-year ingrowth equations. $I_{b}, I_{c}=10$-year ingrowth (number of trees per $b a$ ); $N_{b}, N_{c}=$ number of stems per ha at the beginning of the growth period; $B=$ total basal area at the beginning of the growth period. SE: Standard error; $t$ : Student's $t$ statistic; *, ** and *** indicates significance at 5\%, 1\% and $0.1 \%$ level; $\mathrm{R}^{2}$ : Coefficient of determination; F: F statistic of the regression; dF: Degrees of freedom of residuals; SSR: Sum of squares of regression; SSE: Sum of squares of residuals.

Equations of upgrowth for broadleaved sp.: $\quad U_{b}=\alpha+\beta \cdot B_{b}+\delta \cdot B_{b}{ }^{2}+\varepsilon \cdot B_{c}+\gamma \cdot D_{b}$

\begin{tabular}{|c|c|c|c|c|c|}
\hline & Constant & $\begin{array}{c}\text { Basal area broad. } \\
\left(\mathrm{m}^{2} \cdot \mathrm{ha}^{-1}\right)\end{array}$ & $\begin{array}{c}\text { Basal area sq. } \\
\text { broad. }\end{array}$ & $\begin{array}{c}\text { Basal area conif. } \\
\left(\mathrm{m}^{2} \cdot \mathrm{ha}^{-1}\right)\end{array}$ & Diameter $(\mathrm{cm})$ \\
\hline Coefficient & 0.12421 & -0.00561 & 0.00010846 & -0.00066635 & 0.00003698 \\
\hline SE & 0.00562 & 0.0006988 & 0.00002379 & 0.00018807 & 0.00001135 \\
\hline t & $22.08^{* * *}$ & $-8.03^{* * *}$ & $4.56^{* * *}$ & $-3.54^{* *}$ & $3.26^{* *}$ \\
\hline \multicolumn{6}{|c|}{ Statistical parameters } \\
\hline $\mathrm{R}^{2}$ & $\mathrm{~F}$ & Prob $>F$ & $\mathrm{dF}$ & SSR & SEE \\
\hline 0.07 & 39.25 & $<0.0001$ & 1986 & 0.8129 & 10.2762 \\
\hline
\end{tabular}

Equations of upgrowth for pines: $\quad U_{c}=\alpha+\beta \cdot B_{b}^{2}+\delta \cdot B_{c} \cdot+\varepsilon \cdot B_{c}^{2}+\gamma \cdot D_{c}$

\begin{tabular}{|c|c|c|c|c|c|}
\hline & Constant & $\begin{array}{c}\text { Basal area sq. } \\
\text { broad. }\end{array}$ & $\begin{array}{l}\text { Basal area conif. } \\
\left(\mathrm{m}^{2} \cdot \mathrm{ha}^{-1}\right)\end{array}$ & $\begin{array}{l}\text { Basal area sq. } \\
\text { conif. }\end{array}$ & Diameter $(\mathrm{cm})$ \\
\hline Coefficient & 0.20509 & -0.00006528 & -0.00556 & 0.00005774 & 0.0001318 \\
\hline SE & 0.00686 & 0.00001773 & 0.0005064 & 0.0000023 & 0.00001435 \\
\hline $\mathrm{t}$ & $29.82^{* * *}$ & $-3.68^{* * *}$ & $-10.97^{* * *}$ & $5.64^{* * *}$ & $9.18^{* * *}$ \\
\hline \multicolumn{6}{|c|}{ Statistical parameters } \\
\hline $\mathrm{R}^{2}$ & $\mathrm{~F}$ & Prob $>F$ & $\mathrm{dF}$ & SSR & SEE \\
\hline 0.15 & 124.58 & $<0.0001$ & 2812 & 3.812 & 25.281 \\
\hline
\end{tabular}

Table 3. Upgrowth probability equations. $U_{b}, U_{c}=1$-year upgrowth probability; $D_{b}, D_{c}=$ Diameter $(\mathrm{cm})$ at the beginning of the growth period; $B_{c}, B_{b}=$ Basal area. $\left(m^{2} \cdot h a^{-1}\right)$ at the beginning of the growth period. SE: Standard error; $t$ : Student's t statistic; *, ** and *** indicates significance at 5\%, $1 \%$ and $0.1 \%$ level; $R^{2}$ : Coefficient of determination; F: F statistic of the regression; $d F$ : Degrees of freedom of residuals; SSR: Sum of squares of regression; SSE: Sum of squares of residuals. 


\subsection{Simulations and analysis}

The results of the no-harvest simulation showed that at the beginning the ingrowth of pines decreased slowly while the number of trees of medium size increased due to upgrowth. After four 5-year periods the stand began to close with a high basal area of $37.6 \mathrm{~m}^{2} \mathrm{ha}^{-1}$, a reduced number of pine trees in the $15 \mathrm{~cm}$ diameter class and an accumulation of pines in the $60 \mathrm{~cm}$ diameter class (Fig 3).

The ingrowth of broadleaved species also declined but at a slower rate, leading to an increased share of broadleaved species in the composition of the stand, with $35.2 \%$ of the number of trees and $24.0 \%$ of the total basal area after 20 years.
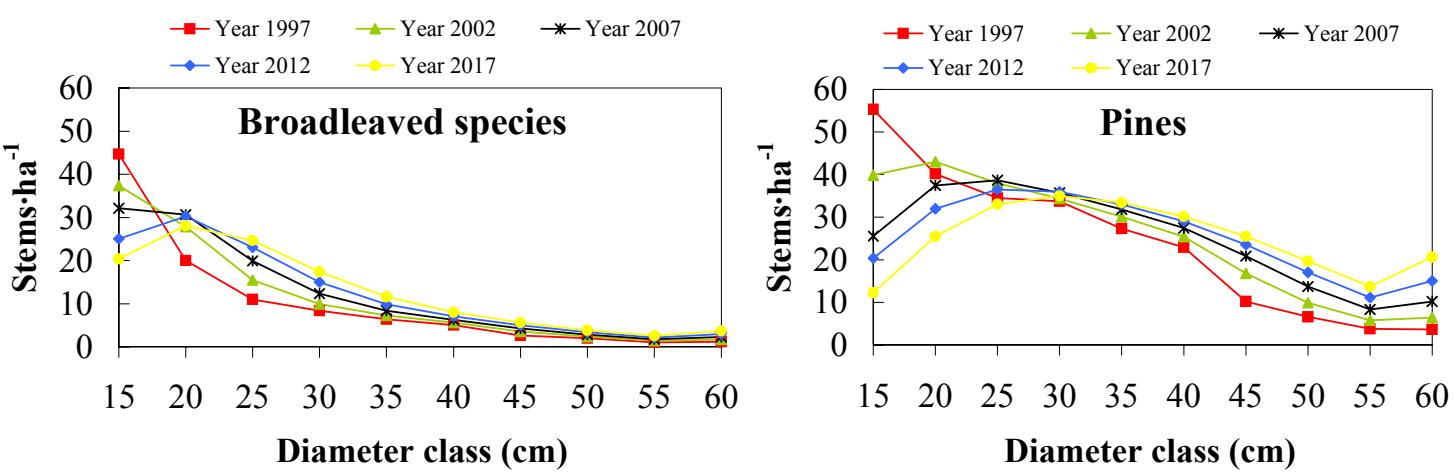

Fig. 3. Diameter distribution in an unmanaged forest after 20 years.

The simulation for the harvest regime in well-stocked stands covered four 5-year periods. The average removed volume per period amounted to $37.1 \mathrm{~m}^{3} \mathrm{ha}^{-1}$ and the productivity is 7.4 $\mathrm{m}^{3} \mathrm{ha}^{-1} \mathrm{yr}^{-1}$ (Table 4). A high proportion of the harvested roundwood belonged to the highest diameter classes, so that $35 \%$ of the pine timber produced could be classified as grade $\mathrm{I}$, or logs with a thin-end diameter greater than $35 \mathrm{~cm}$. 


\begin{tabular}{|c|c|c|c|c|}
\hline $\begin{array}{c}\text { Diameter } \\
\text { class } \\
(\mathrm{cm})\end{array}$ & Pines ha ${ }^{-1}$ & Broadleaved ha ${ }^{-1}$ & $\begin{array}{c}\text { Volume of } \\
\text { pines }\left(\mathrm{m}^{3} \cdot \mathrm{ha}^{-1}\right)\end{array}$ & $\begin{array}{c}\text { Volume of } \\
\text { broadleaved }\left(\mathrm{m}^{3} \cdot \mathrm{ha}^{-1}\right)\end{array}$ \\
\hline 15 & 0.0 & 11.4 & 0.0 & 1.1 \\
\hline 20 & 0.0 & 6.6 & 0.0 & 1.0 \\
\hline 25 & 1.8 & 3.6 & 0.6 & 0.9 \\
\hline 30 & 3.5 & 2.2 & 1.8 & 0.8 \\
\hline 35 & 3.6 & 1.5 & 2.7 & 0.7 \\
\hline 40 & 4.9 & 1.1 & 5.2 & 0.7 \\
\hline 45 & 2.8 & 0.7 & 4.0 & 0.6 \\
\hline 50 & 1.9 & 0.5 & 3.5 & 0.5 \\
\hline 55 & 1.0 & 0.3 & 2.3 & 0.4 \\
\hline 60 & 3.2 & 0.6 & 9.1 & 1.0 \\
\hline Total & 22.7 & 28.5 & 29.2 & 7.9 \\
\hline
\end{tabular}

Table 4. Average number and volume of harvested trees per ha distributed on diameter classes. Average for a 5-year period in the simulation of harvesting well stocked stands.

After 20 years of selection management, a balanced dbh distribution was obtained (Fig. 4) which was characterised by a basal area before harvest of $20 \mathrm{~m}^{2} \mathrm{ha}^{-1}$, of which $16.6 \%$ were broadleaved species, which amounted to $20.7 \%$ of the number of trees.
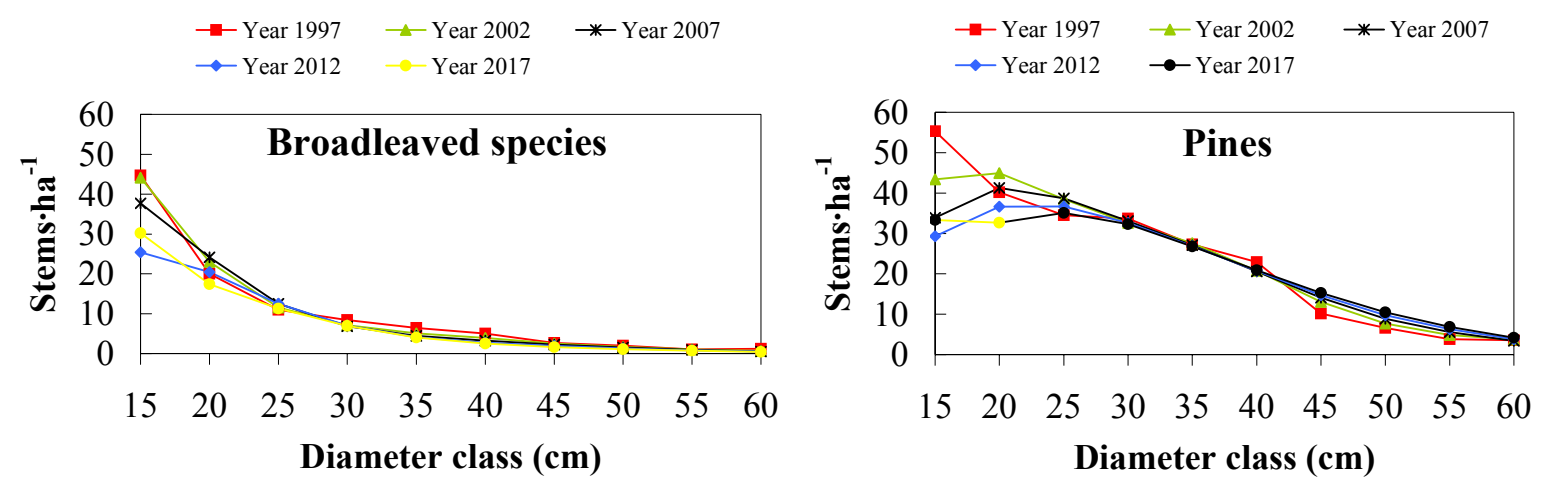

Fig. 4. Development of diameter distributions after 20 years with a harvest cycle of 5 years in a well-stocked stand.

The third simulation involved moderate harvesting to allow the recovery of an understocked stand (Fig. 5). Only 40\% of the annual growth was harvested in this simulation. The result was an increase of the stocking to a basal area of $15 \mathrm{~m}^{2} \mathrm{ha}^{-1}$ or a volume of $114.10 \mathrm{~m}^{3} \mathrm{ha}^{-1}$. The average growth of the stand was $5.36 \mathrm{~m}^{3} \mathrm{ha}^{-1}$. Due to the low harvest yield of 2.35 $\mathrm{m}^{3} \mathrm{ha}^{-1}$, it can be expected that the stand will become fully stocked very soon. 

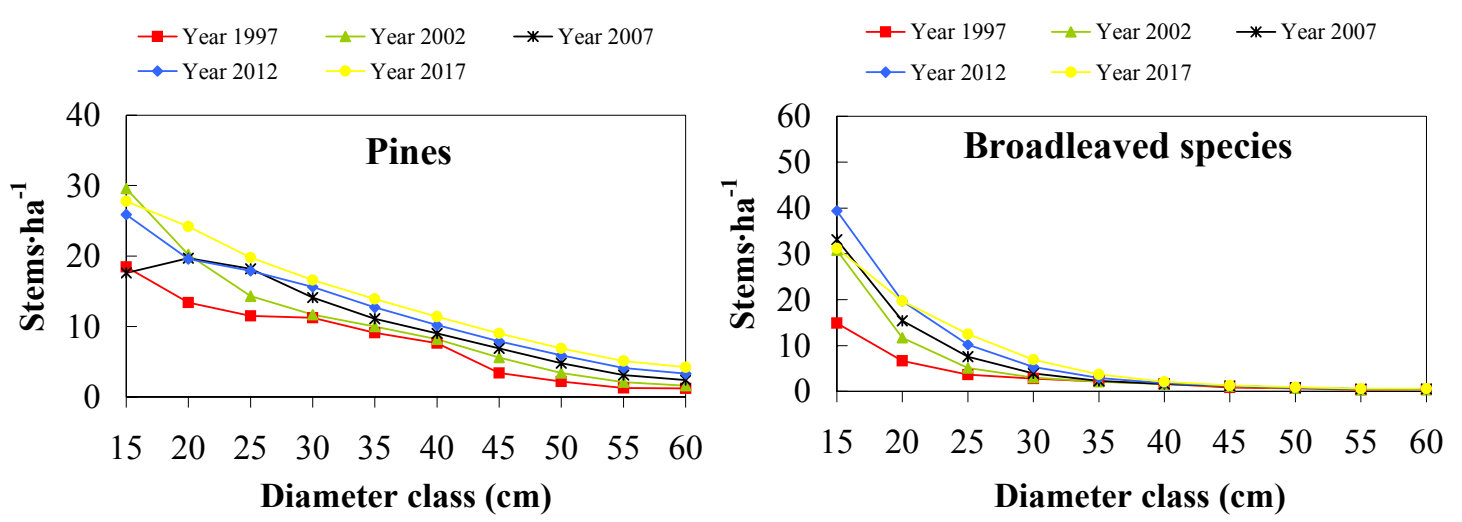

Fig. 5. Development of diameter distributions after 20 years with a harvest cycle of 5 years in an understocked stand.

\section{Discussion}

The potential to commercially grow mixed broadleaved species and maritime pine stands depends mainly on the conifer ingrowth and upgrowth which greatly affects the attainment of a balanced size distribution and adequate growth rates. This essential condition seems to be difficult to achieve with a very light-demanding species like $P$. pinaster. However, the type of uneven-aged management presented in this paper has been suggested and applied in similar forest types involving other pine species (Pinus taeda L., Pinus echinata Mill.) and broadleaved species in the South Eastern USA (Baker and Murphy, 1982; Baker, 1989).

\subsection{The matrix growth model}

The basal area coefficients in the logistic regression for probability of ingrowth have, as expected, positive sign for both broadleaved species and conifers. All coefficients in the equations for probability of ingrowth, except the intercept in the equation for conifers, were significant at least at the $0.01 \%$ level as judged by the Wald $\chi^{2}$ statistic (Hosmer and Lemeshow, 1989). $72 \%$ of the data were successfully identified by the equation for conifers and $63.8 \%$ in case of broadleaved species.

For the ingrowth regression, the coefficients for $\mathrm{N}$ (the number of trees of the same species) had a positive sign. Also the stand basal area had the expected negative sign in the regressions for pines as well as for the broadleaved species. The coefficients of determination $\left(\mathrm{R}^{2}\right)$ were quite low in both the pines and in the broadleaved model, implying that a small percentage of the ingrowth was explained by the model. This may be due to the differences in the harvest selections practiced in the various plots, i.e. to the lack of common management guides. 
The independent variables in the equations estimating the transition probabilities were basal area of conifers and broadleaved sp., squared basal area and diameter (Tab. 2). The coefficient of determination showed that the pine model explains about $15 \%$ of the variation in the upgrowth process whereas in the broadleaved species model this value decreases to $7 \%$. These values are quite low, but similar to the results obtained by other authors who also found that the upgrowth of broadleaved species was more difficult to explain (Buongiorno et al., 1995; Volin et al., 1996).

The probability of mortality is affected by the basal area and the tree size represented by the breast height diameter. In the case of conifers these variables were significant at least at the $0.1 \%$ level as defined by the Wald $\chi^{2}$ statistic and $62.3 \%$ of the data were successfully identified by the model. In the case of broadleaved species the coefficients were significant at least at the $0.001 \%$ level and $59.4 \%$ of the data were successfully identified by the equation. The mortality of broadleaved species seems to be more difficult to explain. This phenomenon has also been observed in other studies (Volin et al., 1996; Rautiainen, 1999).

\subsection{Simulation and analysis}

Based on the results of the simulations, the management regime for mixed, uneven-aged, well stocked stands applied in this paper can be recommended for practical purposes in Galicia. If this harvest pattern were to continue the forest would remain in a balanced situation. The sustainable harvest levels obtained with this regime are lower than those for even-aged stands of pure maritime pine. MAI values of 12 to $15 \mathrm{~m}^{3} \mathrm{ha}^{-1} \mathrm{yr}^{-1}$ can be obtained on medium to good quality sites (Rodríguez, 1995), i.e. in areas where the productivity is comparable to the forest analysed in this study. Nevertheless it is important to point out the potential for highvalue timber production under uneven-aged selective management. For example, the proportion of grade I timber - a valuable class that is in high demand by the local sawmilling and veneer industry - reaches $40 \%$, which is much higher than the $16 \%$ obtained for an evenaged saw timber regime (Rodríguez et al., 2000). Also, the high degree of competition and the lack of light may favour selfpruning and reduce the low-quality juvenile core in the pines.

A fully stocked stand may be managed in the selection system producing timber every 5 years. This is not possible in an understocked stand where a decision has to be taken whether to rehabilitate the existing forest or prepare the site and regenerate the forest to obtain a 
satisfactory stocking (Baker, 1989). A forest with a basal area before harvest of $11 \mathrm{~m}^{2} \mathrm{ha}^{-1}$ could be considered as understocked.

Simulation results show the need of improvement cuts, concentrated on broadleaved species, and removing only a small part of the annual growth, these results are comparable with those obtained by Baker (1986) in the South-Eastern United States who could show rapid rehabilitation of an uneven-aged pine-hardwood forest with an average growth of 6.2 $\mathrm{m}^{3} \mathrm{ha}^{-1} \mathrm{yr}^{-1}$ during the first 14 years of rehabilitation management.

If the no-harvesting management option were to continue, the stand structure would change to a prevalence of broadleaved species with virtually no pine ingrowth. This prediction proves that model performance is consistent with the expected natural evolution observed in Galicia. Similar results were also obtained for the Italian Alps by Volin et al. (1996) and from the French Jura by Buongiorno et al. (1995).

In conclusion, a selection silviculture aiming to maintain an adequate proportion of broadleaved species in the stand, thus allowing sufficient regeneration and ingrowth of pines and controlling undesirable eucalypts, may be a practical alternative in Galicia, if the aim is to establish a mixed continuous cover forest. The presence of eucalyptus trees makes the management of the stands very difficult, since the growth patterns, reproduction methods and usual harvest ages are very different in this species. Normally pine and oak are dominated by the Eucalypts, with poor growth of the suppressed trees. The continuous cover forest management has also other indirect benefits, such as the protection against soil erosion, the improvement of landscape aesthetics, the reduction of negative impacts associated with forest fragmentation, the improvement of wildlife habitat or the protection against natural disturbances (Matthews, 1989; Baker et al., 1991; Schulte and Buongiorno, 1998). However, the cost of tree selection and logging are generally higher, more technical expertise is required to regulate harvests and greater damage to residual trees can be expected. In the future, more information should be collected from permanent plots representing a variety of silvicultural treatment options.

\section{Acknowledgements}

The research reported in this study was supported by "Fundación Pedro Barrie de la Maza" and by the Spanish Education Ministry within the program of grants for stays of spanish 
researchers in foreign institutions. We thank Prof. Dr. Klaus von Gadow and Prof. Dr.

Andres Kiviste for their advice and valuable comments.

\section{References}

Baker, J. B., 1986: The Crossett farm forestry forties after 41 years of selection management. South. J. Appl. For. 10: 233-237.

Baker, J. B., 1989: Recovery and development of understocked loblolly-shortleaf pine stands. South. J. Appl. For. 13: 132-139.

Baker, J. B. and Murphy, P. A., 1982: Growth and yield following four reproduction cutting methods in loblolly-shortleaf pine stands. South. J. Appl. For. 6: 66 - 74.

Baker, J. B., Guldin, J. M. and Guldin, R. W., 1991: Natural regeneration methods for loblolly and shortleaf pines. For. Farmer 50: $59-63$.

Buongiorno, J., Peyron, L., Houllier, F. and Bruciamacchie, M., 1995: Growth and management of mixed-species, uneven-aged forest in the French Jura: Implications for economic returns and tree diversity. For. Sci. 41: 397-429.

Chang, S. J., 1990: An economic comparison of even-aged and uneven-aged management of southern pines in the mid-South. In Hickman, C.A. (Ed.), Proc. of the Southern Forest Economics Workshop on Evaluating Even- and All-Aged Timber Management Options for Southern Forest Lands, March 29-30 1990, Monroe, LA. GTR SO-79. USDA For. Serv., Southern Exp. Stn., New Orleans, LA. pp. 45-52.

DGCONA, 2000: Avance del Tercer inventario forestal nacional en Galicia. Ministerio de Medio Ambiente. Madrid. (In Spanish).

Gadow, K. v. and Hui, G. Y., 1999: Modelling forest development. Kluwer Academic Publisher, Dordrecht: 213 p. ISBN 0-7923-5488-5.

Hamilton, D.A., 1986: A logistic model of mortality in thinned and unthinned mixed conifers stands of Northern Idaho. For. Sci. 32: 989 - 1000.

Hosmer, D.W. and Lemeshow, W., 1989: Applied logistic regression. Wiley, 307 p. New York. ISBN 0-471-61553-6.

ICONA, 1993: Segundo inventario forestal nacional 1986-1995. Galicia. La Coruña. (In Spanish).

ICONA, 1993: Segundo inventario forestal nacional 1986-1995. Galicia. Pontevedra. (In Spanish).

Loureiro, A. M., 1991: Apuntamentos de silvicultura (8 capítulos). Universidade de Trás-osmontes e Alto Douro. Vilareal. Portugal. (In Portuguese).

Matthews, J. D., 1989: Silvicultural Systems. Oxford Universty Press, Oxford, 284 p.

Molina, F., 1988: Los bosques de Galicia. Actualidad Forestal de Galicia. N 106-107 EneroJunio. pp.1-5. (In Spanish).

Rautiainen, O., 1999: Growth dynamics and management of Shorea Robusta forests in Southern Nepal. Ph D. Dissertation. Faculty of Forestry. University of Joensuu: 111 p. ISBN 951-708-810-8.

Redmond, C. H., and Greenhalgh, R., 1990: An economic analysis of even-aged versus uneven-aged management on nonindustrial private land in southern Arkansas. In Hickman, C.A. (Ed.), Proc. of the Southern Forest Economics Workshop on Evaluating Even- and All-Aged Timber Management Options for Southern Forest Lands, March 29-30 1990, Monroe, LA. GTR SO-79. USDA For. Serv., Southern Exp. Stn., New Orleans, LA. pp.69-79.

Rodríguez, R., 1995: Crecimiento y producción de masas forestales regulares de Pinus pinaster Ait. en Galicia. Alternativas selvícolas posibles. Ph D. Dissertation. Escuela Técnica Superior de Ingenieros de Montes. Madrid, 297 p. (In Spanish). 
Rodríguez, R., Alvarez, J. G. and Schröder, J., 2000: Simulation and comparison of silvicultural alternatives for even-aged Pinus pinaster stands in Galicia (Northwestern Spain). Ann. For. Sci. 57: 747-754.

Romero, A., 1992: El sector forestal en Galicia. Economía Política Forestal. Xunta de Galicia. Santiago de Compostela. pp. 113-132. (In Spanish).

Ruíz Zorrilla, P., 1981: Notas para una historia del pino en Galicia. Publicaciones del Centro Forestal de Lourizán, Pontevedra. (In Spanish).

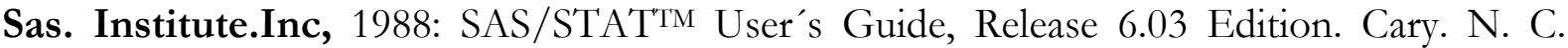
USA.

Schulte, B. J. and Buongiorno, J., 1998: Effects of uneven-aged silviculture on the stand structure, species composition, and economic returns of loblolly pine stands. For. Ecol. Manage. 111:83-101.

Volin, C. and Buongiorno, J., 1996: Effects of alternative management regimes on forest stand structure, species composition and income: A model for the Italian Dolomites. For. Ecol. Manage. 87:107-125.

Woollons, R. C., 1998: Even-aged stand mortality estimation through a two-step regression process. For. Ec. Man. 105: 189-195.

Xunta de Galicia, 1992: Plan forestal de Galicia. Síntesis. Consellería de Agricultura Gandería e Montes. 



\section{Veröffentlichung V}

Optimal residual growing stock and cutting cycle in mixed uneven-aged maritime pine stands in northwestern Spain

SÁNCHEZ Orois, S., ChANG, S. J. Und GADOW, K. V.

Angenommen (im Druck):

Forest Policy and Economics 



\title{
Optimal residual growing stock and cutting cycle in mixed uneven-aged maritime pine stands in Northwestern Spain
}

\author{
SOFÍA SÁNCHEZ OROISa, SUN JOSEPH CHANG ${ }^{\mathrm{b}}$, KLAUS VON GADOWc \\ a, Institut für W aldinventur und Waldwachstum. Georg-August-Universität Göttingen \\ Büsgenweg 5, 37077 Göttingen. Germany \\ ${ }^{b}$ Chool of Forestry, Wildlife, and Fisheries. Louisiana State University \\ Baton Rouge, LA 70803-6202. USA
}

\begin{abstract}
This study analyses two important phases of the Continuous Cover Forestry (CCF) management. The first phase involves the determination of the optimum cutting cycle and residual growing stock level for mixed uneven-aged Pinus pinaster Ait. stands in Galicia (NW Spain). The Faustmann formula to calculate the Land Expectation Value (LEV) was used for the economic evaluation of the different management options. In addition, sensitivity analysis was carried out assuming varying price levels and discount rates. The second phase deals with a comparison between CCF management and Rotation Forest Management (RFM). The land expectation values for four commonly used rotation forest management practices were calculated. The results of the comparison indicate that on low sites $(\mathrm{SI} \leq 13) \mathrm{CCF}$ management is superior, whereas on high sites (SI>13) RFM gives higher returns.
\end{abstract}

Keywords: CCF management, rotation forest management, growing stock, cutting cycle, maritime pine, forest value, land expectation value, Faustmann. 


\section{Introduction}

Using a simple classification we may distinguish between even-aged rotation forest management (RFM) systems and uneven-aged continuous cover forestry (CCF) systems. Intensive RFM with standard silviculture are found throughout the world and are characterized by selective removals and are most frequently found in densely populated regions and in some tropical forests. Private non-industrial forest owners and government agencies are increasingly interested in CCF management (Hill, 1992; Gadow and Puumalainen, 2000).

The traditional type of forest management applied by the Forest Service in the maritime pine stands in Galicia (NW Spain) is a RFM system which is characterized by periodic replanting or natural regeneration followed by a succession of thinnings and a clearfelling when the forest has reached rotation age. The almost 400000 ha covered by pure maritime pine stands in Galicia are managed according to this system.

A special type of pine forest with a mixture of broadleaved species and maritime pines of different ages has developed in northwestern Spain as a result of the management applied by the forest owners, which is very different from that applied by the Forest Service. The total area of these forests amounts to 87000 ha in the coastal region of Galicia. These forests are normally small non-industrial private forest, covering less than 1 ha on average. The management involves normally a kind of selective high thinning of the stands, where only the best and the largest trees are harvested whenever cash income is required by the owners. The residual trees are usually of poor quality and inferior growth (Molina, 1988), resulting in an uneven-aged forest, which is understocked and in many cases lacking commercially valuable trees (Romero, 1992).

The potential for active management of these forest lands has not been explored. Recently Sánchez and Rodríguez (2002) developed a matrix growth model allowing an evaluation of the growth and management options for these forests which are potential candidates to be managed as CCF systems. One of the attractions of CCF management is the fact that a continuous tree cover is maintained. Only single trees or at most small patches of trees are harvested at a given point in time, thus protecting the forest against soil erosion, often improving the landscape aesthetics, and reducing the negative effects of habitat fragmentation (Guldin, 1996; Saunders et al., 1991). However, CCF management does have its drawbacks. It is deemed to be more difficult to apply, both at the stand and at the forest 
level than RFM systems (Hotvedt and Ward, 1990). In CCF management harvesting costs per unit of volume are greater (Kluender et al., 1998), and there is often damage to residual trees and high -grading (Stokes et al., 1993).

Under certain conditions selective harvesting systems may be financially superior to RFM for landowners (Redmond and Greenhalgh, 1990). The uneven-aged forest produces small but frequent revenues while the revenues in even-aged forests are large but infrequent. At high interest rates or long rotation ages, the large but infrequent revenue will be heavily discounted, thus making it less favorable than the most frequent but smaller revenues from the uneven-aged forest (Chang, 1990).

The aim of this paper is to determine the optimal growing stock and cutting cycle in mixed uneven-aged stands of maritime pine using financial criteria and to compare the CCF management against that of RFM.

\section{Methodology and theoretical approach}

The data used in this study are derived from 213 circular sample plots selected among the available 4700 plots of the Spanish National Inventory which are located in the provinces of A Coruña and Pontevedra, in the autonomous region of Galicia. The selected plots are characterised by i) a large diameter range (with at least 5 diameter classes with a class width of $4 \mathrm{~cm}$ represented), ii) the absence of eucalypts and iii) a mixture of broadleaved species (mainly Quercus robur) and maritime pine with at least 10\% of stems in each of the two groups.

A matrix stand growth model in which the growth parameters are functions of the stand density, expressed by basal area, was used to simulate stand development in accordance with the approach proposed by Buongiorno et al. (1995):

$$
\boldsymbol{y}_{t+1}=\boldsymbol{G} \cdot\left(\boldsymbol{B}_{t}\right) \cdot\left(\boldsymbol{y}_{t}-\boldsymbol{h}_{t}\right)
$$

where:

$\mathbf{y}_{\mathbf{t}}=\left[\mathrm{y}_{\mathrm{itt}}\right]-$ Column vector representing the state of the stand at time $\mathrm{t} ; \mathrm{i}=1 \ldots \mathrm{n}$, indicates the tree species; $j=1 . . m$ the diameter size class.

$\mathbf{h}_{\mathbf{t}}=\left[\mathrm{h}_{\mathrm{ijt}}\right]-$ Column vector of the number of trees of species $\mathrm{i}$ and size class $\mathrm{j}$ harvested at time $t$.

$\mathbf{G}=$ Growth matrix as function of the basal area $\mathbf{B}$. Its parameters represent the effects of stand density and number of trees on ingrowth, as well as the 
probabilities that a tree stays in a size class or moves into a larger class during the interval $t$ to $\mathrm{t}+1$.

More details about the methods used to fit the parameters of the model are described in Sánchez and Rodríguez (2002).

Different management options analysing 3, 4, 5, 6 and 7-year cutting cycles and several residual growing stock levels were generated using the matrix growth model. A comparative analysis of the land expectation value for each management option was done to determine the optimal stocking and cutting cycle using the classical Faustmann land expectation value formula (Faustmann, 1849).

The management options were generated for a stand the initial species composition and other attributes of which were equal to the average composition of the 213 mixed plots from the last survey carried out in Galicia. The stand has a basal area of $21.8 \mathrm{~m}^{2} \cdot \mathrm{ha}^{-1}$ and a stocking of 362.7 stems $^{* h^{-1}}$, of which $67 \%$ are pines and 33\% broadleaved species, mainly oak. All together 20 options were simulated, involving all possible combinations of 5 cutting cycles (3, 4, 5, 6 and 7-year cutting cycle) and 4 different growing stock levels with a residual basal area of $10,13,15$ and $17 \mathrm{~m}^{2 \cdot}$ ha $^{-1}$ giving a residual growing stock volume of 73, 103, 119 and 133 $\mathrm{m}^{3} \cdot \mathrm{ha}^{-1}$ respectively.

Table 1 presents the harvest volume for each management option classified accordingly the wood quality. In order to calculate the land expectation value for each option, stumpage prices per $\mathrm{m}^{3}$ were estimated for each of the above defined timber grades in accordance with the timber auction prices realised by the forest administration of Galicia during previous years $\left(\right.$ Grade I $=90 € / \mathrm{m}^{3}$, Grade II $=60 € / \mathrm{m}^{3}$, Grade III $=18 € / \mathrm{m}^{3}$ and Broadleaved species $\left.=21 € / \mathrm{m}^{3}\right)$. 


\begin{tabular}{|c|c|c|c|c|c|c|}
\hline \multirow{2}{*}{$\begin{array}{c}\text { Residual } \\
\text { growing stock } \\
\left(\mathrm{m}^{3} \mathrm{ha}^{-1}\right)\end{array}$} & \multirow{2}{*}{$\begin{array}{l}\text { Wood quality } \\
\text { class }\end{array}$} & \multicolumn{5}{|c|}{ Cutting cycle (years) } \\
\hline & & 3 & 4 & 5 & 6 & 7 \\
\hline \multirow{5}{*}{73} & Grade I & 7.43 & 10.69 & 10.62 & 10.59 & 10.17 \\
\hline & Grade II & 3.81 & 4.47 & 7.45 & 8.71 & 9.43 \\
\hline & Grade III & 2.53 & 3.61 & 5.78 & 10.76 & 12.02 \\
\hline & Broadleaved sp & 2.52 & 4.05 & 6.71 & 7.62 & 9.52 \\
\hline & Total Volume & 16.29 & 22.82 & 30.56 & 37.68 & 41.14 \\
\hline \multirow{5}{*}{103} & Grade I & 12.18 & 14.40 & 18.97 & 19.70 & 19.41 \\
\hline & Grade II & 3.01 & 5.99 & 9.58 & 8.43 & 9.51 \\
\hline & Grade III & 2.20 & 4.53 & 6.78 & 10.23 & 10.60 \\
\hline & Broadleaved sp & 1.91 & 3.34 & 4.29 & 6.20 & 7.46 \\
\hline & Total Volume & 19.30 & 28.26 & 39.61 & 44.57 & 46.98 \\
\hline \multirow{5}{*}{119} & Grade I & 13.66 & 16.81 & 22.44 & 22.41 & 22.03 \\
\hline & Grade II & 3.60 & 5.98 & 7.70 & 8.75 & 12.36 \\
\hline & Grade III & 2.53 & 4.46 & 5.85 & 10.09 & 10.86 \\
\hline & Broadleaved sp & 1.85 & 3.48 & 4.28 & 6.36 & 7.67 \\
\hline & Total Volume & 21.64 & 30.73 & 40.28 & 47.62 & 52.91 \\
\hline \multirow{5}{*}{133} & Grade I & 14.80 & 19.19 & 24.29 & 25.45 & 26.27 \\
\hline & Grade II & 5.31 & 5.97 & 8.55 & 10.37 & 10.48 \\
\hline & Grade III & 3.21 & 4.79 & 5.58 & 7.81 & 10.15 \\
\hline & Broadleaved sp & 1.66 & 2.89 & 5.11 & 7.64 & 8.97 \\
\hline & Total Volume & 24.98 & 32.83 & 43.52 & 51.27 & 55.87 \\
\hline
\end{tabular}

Table 1. Periodic harvest volume $\left(\mathrm{m}^{3} \mathrm{ha}^{-1}\right)$ for each cutting cycle and residual growing stock level. Grade I: logs with a small-end diameter larger than $35 \mathrm{~cm}$. Grade II: logs with a small-end diameter between 22 and $35 \mathrm{~cm}$. Grade III: Logs with a small-end diameter less than $22 \mathrm{~cm}$.

To calculate the land expectation value for the different options, we followed the approach first suggested by Chang (1981) for uneven-aged forests.

$$
\begin{aligned}
\operatorname{Max} F V & =V(S)-v(g)-k+\left[V(Q(t, g)-v(g)-k]\left(e^{-r t}+e^{-2 r t}+e^{-3 r t}+\ldots\right)\right. \\
& =V(S)-v(g)-k+\frac{V(Q(t, g)-v(g)-k}{e^{r t}-1} \\
& =V(S)+\frac{V(Q(t, g)-v(g)-k}{e^{r t}-1}-v(g)-k
\end{aligned}
$$

where:

FV is the forest value of the uneven-aged forest;

$\mathbf{V ( S )}$ is the stumpage value of the existing uneven-aged stand with an existing volume of $\mathrm{S}$;

$\mathbf{V}(\mathbf{Q}(\mathbf{t}, \mathbf{g}))$ represents the stumpage value associated with a stand volume $\mathrm{Q}$ result from an initial residual growing stock of $g$ and a cutting cycle of $t$ years;

$\mathbf{v}(\mathrm{g})$ is the value of the residual growing stock $g$;

$\mathbf{k}$ is the fixed cost associated with each harvest;

$\mathbf{r}$ is the interest rate. 
Given the fact that the existing stand volume $S$ and value $V(S)$ are fixed, the maximization of the forest value of equation 2 is essentially the maximization of the classical land expectation value portion of the forest value (Chang, 1981)

$$
L E V=\frac{V(Q(t, g))-v(g)-k}{e^{r t}-1}-v(g)-k
$$

\section{Results}

\subsection{Optimal cutting cycle and residual growing stock}

Table 2 presents the harvest values for the different combinations of residual growing stock and cutting cycle and the calculated LEV for a discount rate $\mathrm{r}$ of 0.04 , and a fixed cost $\mathrm{K}$ of $1.5 € \cdot \mathrm{ha}^{-1}$. For the given stumpage prices, the optimal combination of $\mathrm{t}$ and $\mathrm{g}$ is a cutting cycle of 5 years with a residual growing stock of $133 \mathrm{~m}^{3}$ per ha at the beginning of the cutting cycle. The resulting LEV value is $6083.82 €$ per hectare.

\begin{tabular}{crcccc}
\hline \multirow{2}{*}{$\begin{array}{c}\text { Residual } \\
\text { growing stock } \\
\left(\mathrm{m}^{3} \cdot \mathrm{ha}^{-1}\right)\end{array}$} & $\mathbf{3}$ & $\mathbf{4}$ Cutting cycle (years) \\
\cline { 2 - 6 } & 995.65 & 1380.60 & 1648.18 & 1829.50 & $\mathbf{7}$ \\
\hline 73 & 1356.24 & 1807.10 & 2494.00 & 2593.50 & 2697.68 \\
103 & 1529.80 & 2024.78 & 2676.86 & 2857.64 & 3080.28 \\
119 & 1743.07 & 2232.36 & 2906.50 & 3213.62 & 3364.05 \\
133 & \multicolumn{5}{c}{ Harvest value $€ \cdot \mathrm{ha}^{-1}$} \\
\hline 73 & 4492.41 & 4643.17 & 4132.45 & 3434.15 & 2563.13 \\
103 & 5116.30 & 4896.89 & 5748.39 & 4046.42 & 2732.92 \\
119 & 5537.14 & 5211.01 & 5633.86 & 4079.76 & 3078.18 \\
133 & 5971.10 & 5820.12 & $\mathbf{6 0 8 3 . 8 2}$ & 4804.89 & 3369.13 \\
\hline
\end{tabular}

Table 2. Harvest value for each cutting cycle and results of the analysis for the $L E V$ for $r=4 \%$ and $K=$ $1,5 € \cdot h a^{-1}$.

\subsubsection{The effect of raising and lowering the discount rate}

The results of an analysis using different discount rates is presented in Table 3. When a discount rate of $1 \%$ was applied, both the optimal cutting cycle and residual growing stock level remain unchanged. 


\begin{tabular}{cccccc}
\hline $\begin{array}{c}\text { Residual } \\
\begin{array}{c}\text { growing stock } \\
\left(\mathrm{m}^{3} \mathrm{ha}^{-1}\right)\end{array}\end{array}$ & $\mathbf{3}$ & $\mathbf{4}$ & $\mathbf{5}$ & $\mathbf{6}$ & $\mathbf{7}$ \\
\cline { 2 - 6 } & & & & & \\
\hline$r=1 \%$ & 29338.70 & 30487.51 & 28812.08 & 26256.77 & 22846.22 \\
\hline 73 & 38974.65 & 38733.85 & 43104.76 & 36407.59 & 31222.07 \\
103 & 43733.18 & 43127.28 & 45730.85 & 39738.72 & 36011.32 \\
119 & 47421.17 & 47626.45 & $\mathbf{4 9 6 2 2 . 5 4}$ & 44908.27 & 39337.71 \\
\hline 133 & & & & & \\
\hline$r=7 \%$ & 949.32 & $\mathbf{9 6 2 . 9 0}$ & 624.35 & 197.13 & -306.26 \\
73 & 288.09 & 78.48 & 438.36 & -543.49 & -1297.35 \\
103 & 90.38 & -188.31 & -65.74 & -977.88 & -1580.77 \\
119 & 60.31 & -133.15 & -105.00 & -883.12 & -1719.23 \\
\hline 133 & &
\end{tabular}

Table 3. Values of LEV using discount rates of $1 \%$ and $7 \%$.

In the case of a higher discount rate $r=7 \%$ the best residual growing stock was only $73 \mathrm{~m}^{3} \cdot \mathrm{ha}^{-1}$ and the best cutting cycle only 4 years. When discount rates of more than $7.6 \%$ were applied, the optimum cutting cycle decreased to 3 years. As the interest rate increases, the opportunity costs associated with maintaining the residual stand are higher and for this reason the residual growing stock is lower. Likewise, when raising the rate of interest, the shorter cutting cycles are preferred because of the earlier revenues.

\subsubsection{The effect of lowering the stumpage price}

Since CCF management typically realize a stumpage price that is lower than that realized under even-aged management, in this section we explore the effects of lower stumpage prices on management decisions under 4, 1 and 7\% interest rate. As shown in Table 4, with a 20\% lower stumpage prices, the land expectation values in all cases are lower by slightly more than $20 \%$. This is because the fixed cost in the formula does not change. More importantly, all the optimal combinations of cutting cycle and residual growing stock levels remain the same. 


\begin{tabular}{ccrrrr}
\hline \multirow{2}{*}{$\begin{array}{c}\text { Residual growing } \\
\text { stock }\left(\mathrm{m}^{\mathbf{3}} \mathrm{ha}^{-1}\right)\end{array}$} & $\mathbf{3}$ & $\mathbf{4}$ & $\mathbf{5}$ & $\mathbf{6}$ & $\mathbf{7}$ \\
\cline { 2 - 6 }$r=4 \%$ & & & & \\
\hline 73 & 3591.27 & 3712.50 & 3304.30 & 2745.92 & 2049.27 \\
103 & 4090.39 & 3915.49 & 4597.06 & 3235.73 & 2185.11 \\
119 & 4427.06 & 4166.78 & 4505.43 & 3262.40 & 2461.31 \\
133 & 4774.23 & 4654.06 & $\mathbf{4 8 6 5 . 4 0}$ & 3842.51 & 2694.08 \\
\hline$r=1 \%$ & & & & \\
\hline 73 & 23460.81 & 24382.35 & 23043.51 & 21000.26 & 18272.54 \\
103 & 31169.57 & 30979.43 & 34477.66 & 29120.92 & 24973.22 \\
119 & 34976.40 & 34494.18 & 36578.53 & 31785.83 & 28804.62 \\
133 & 37926.78 & 38093.51 & $\mathbf{3 9 6 9 1 . 8 8}$ & 35921.47 & 31465.73 \\
\hline$r=7 \%$ & 757.87 & $\mathbf{7 6 9 . 0 9}$ & 498.46 & 156.83 & -245.78 \\
\hline 73 & 228.89 & 61.55 & 349.67 & -435.66 & -1038.65 \\
103 & 70.72 & -151.88 & -53.61 & -783.18 & -1265.39 \\
133 & 46.67 & -107.75 & -85.02 & -707.37 & -1376.16 \\
\hline
\end{tabular}

Table 4. Values of LEV when stumpage prices are 20\% lower using discount rates of $4 \%, 1 \%$ and $7 \%$.

\subsection{Comparison with the Rotation Forest Management}

Prior to the conversion from rotation to continuous cover forest management, it is important to determine the difference between the land expectation values under both management regimes. For rotation forest management, the objective is to maximize

$L E V=\frac{V(t)+\sum_{i=1}^{n} H_{i} e^{r\left(t-t_{i}\right)}-C e^{r t}}{e^{r t}-1}$

where:

LEV is the land expectation value;

$\mathbf{V}(\mathbf{t})$ is the stumpage value of a t-year-old even-aged stand;

$\mathbf{H}_{\mathbf{i}}$ is the thinning income from the $i$ th thinning;

C is the regeneration cost;

$\mathbf{r}$ is the interest rate.

Since both rotation forest management and continuous cover forestry systems attempt to maximize their respective land expectation value, they provide the basis for economic valuation and comparison between the two management systems.

Rotation forest management under two site indices, that of 16 and 13 (base age of 20), corresponding to the conditions common in the coastal area of Galicia (Rodríguez et al., 
1993; Schröder, 2000), were considered. Two commonly practiced management alternatives, one more intensive and the other less intensive, were examined. Given a site index of 16, a more intensively managed stand, with thinnings from below at age 12, 17, 22, 27 and final harvest at age 35 would realize a land expectation value of $9049.63 €$ per hectare. The less intensive management alternative, with thinnings at age 13,18, 23 and final harvest at age 35, would realize a land expectation value of $7948.23 €$ per hectare. Given a site index of 13, a more intensively managed stand, with thinnings from below at age 12, 17, 22, 27, and final harvest at age 35, would realize a land expectation value of $6030.22 €$ per hectare. The less intensive management alternative, with thinnings at age 15,20,25, and final harvest at age 35, would realize a land expectation value of $5646.38 €$ per hectare

\begin{tabular}{|c|c|c|c|c|c|}
\hline \multicolumn{3}{|c|}{ More intensive management } & \multicolumn{3}{|c|}{ Less intensive management } \\
\hline Age & $\begin{array}{l}\text { Management } \\
\text { activity }\end{array}$ & $\begin{array}{c}\text { Revenue or } \\
\text { expense }\left(€ \cdot \mathrm{ha}^{-1}\right)\end{array}$ & Age & $\begin{array}{l}\text { Management } \\
\text { activity }\end{array}$ & $\begin{array}{c}\text { Revenue or } \\
\text { expense }\left(€ \cdot \mathrm{ha}^{-1}\right)\end{array}$ \\
\hline \multicolumn{6}{|c|}{$S I=16$} \\
\hline 0 & Regeneration & -2000.00 & 0 & Regeneration & -1800.00 \\
\hline 12 & $1^{\text {st }}$ Thinning & 1605.96 & 13 & $1^{\text {st }}$ Thinning & 1067.02 \\
\hline 17 & $2^{\text {nd }}$ thinning & 905.46 & 18 & $2^{\text {nd }}$ thinning & 962.86 \\
\hline 22 & $3^{\text {td }}$ thinning & 503.03 & 23 & $3^{\text {rd }}$ thinning & 1750.59 \\
\hline 27 & $4^{\text {th }}$ thinning & 768.09 & --- & --- & --- \\
\hline \multirow[t]{2}{*}{35} & Final harvest & 27964.08 & 35 & Final harvest & 24280.67 \\
\hline & $\mathrm{LEV}$ & 9049.63 & & $\mathrm{LEV}$ & 7948.23 \\
\hline \multicolumn{6}{|c|}{$S I=13$} \\
\hline 0 & Regeneration & -2000.00 & 0 & Regeneration & -1800.00 \\
\hline 12 & $1^{\text {st }}$ Thinning & 1405.22 & 15 & $1^{\text {st }}$ Thinning & 967.28 \\
\hline 17 & $2^{\text {nd }}$ thinning & 803.68 & 20 & $2^{\text {nd }}$ thinning & 818.25 \\
\hline 22 & $3^{\text {rd }}$ thinning & 417.63 & 25 & $3^{\text {rd }}$ thinning & 1054.60 \\
\hline 27 & $4^{\text {th }}$ thinning & 467.23 & --- & --- & --- \\
\hline \multirow[t]{2}{*}{35} & Final harvest & 20010.83 & 35 & Final harvest & 19333.24 \\
\hline & LEV & 6030.22 & & LEV & 5646.38 \\
\hline
\end{tabular}

Table 5. Revenues and expenses for even-aged plantations and land expectation values under different management alternatives.

\section{Discussion and conclusions}

If the uneven-aged stand has a site index of 16, rotation forest management is far superior to CCF management. The land expectation value under intensive rotation forest management at 9049.63€ per hectare is nearly 1.5 times that under CCF management. On the other hand, if the uneven-aged stand has an even-aged site index of 13, the CCF management is then the preferred choice. The land expectation value under continuous cover forestry management at 6083.82€ per hectare is slightly larger than that under both rotation forest management alternatives. 
It should also be pointed out that if the stumpage prices realized under CCF management will be lower due to poor log quality, than those achieved in rotation management, then CCF is likely to be economically uncompetitive. This points out the need for the CCF management to produce high quality logs to compensate for the higher logging cost. Given that most of the existing uneven-aged stands have been high graded over the years, bringing them under active management would, over time, improve stand quality and eventually meet the challenge of producing high quality logs.

From an economic point of view, for low sites $(\mathrm{SI} \leq 13)$ the results obtained above suggest that the uneven-aged stand should be maintained as such; the even-aged stands should be converted to uneven-aged stands. On the other hand, on high sites (SI>13) the even-aged stands should not be converted to uneven-aged stands. Earlier studies (Chang 1981, 1990) have shown that low stumpage price and high interest rate favor CCF management. From this study, we add that low quality sites also favor CCF management. Taken together, low stumpage prices, high interest rates, and poor site quality would suggest that CCF management is favored in challenging and less profitable situations.

When even-aged stands in high sites are being considered for conversion to unevenaged stands, the forest owners and managers must have a clear idea of what non-timber benefits they are getting from the conversion decision. For example, on sites with a site index of 16, these benefits must be greater than 2965.81€ (9049.83-6083.62) per hectare in present value or $118.63 €$ per hectare in permanent annual income equivalent to justify such an action. Far too often, conversion decisions are made without asking such tough questions.

This paper does not deal with the evaluation of transformation paths from RFM to CCF management, which was the topic of the IUFRO Conference held in Edinburgh in September 1999 and which was explored by some researchers (Buongiorno, 2001; Knocke and Plusczyk, 2001; Hanewinkel and Pretzsch, 2001). This is a complex topic which requires further improvement of the growth models and a better understanding of the dynamics of selective harvesting and subsequent growth in the uneven-aged forests. An important task for future forest research in Galicia would be to analyse and propose specific transformation strategies for the even-aged pine stands. It would also be interesting and necessary to consider not only the financial criteria, but the environmental and social values of the CCF management systems. 


\section{Acknowledgements}

We thank two anonymous reviewers for their valuable comments. The research reported in this paper was partially funded by the "Fundación Caixa Galicia".

\section{References}

Buongiorno, J., Peyron, L., Houllier, F. and Bruciamacchie, M., 1995. Growth and management of mixed-species, uneven-aged forest in the French Jura: Implications for economic returns and tree diversity. Forest Science 41:397-429.

Chang, S. J., 1981. Determination of the Optimal Growing Stock and Cutting Cycle for an Uneven-Aged Stand. Forest Science 27(4):739-744.

Chang, S. J., 1990. An economic comparison of even-aged and uneven-aged management of southern pines in the mid-South. In: Hickman, C.A. (Ed.), Proc. of the Southern Forest Economics Workshop on Evaluating Even- and All-Aged Timber Management Options for Southern Forest Lands, March 29-30 1990, Monroe, LA. GTR SO-79. USDA For. Serv., Southern Exp. Stn., New Orleans, LA. pp. 45-52.

Faustmann, M., 1849. Berechnung des Wertes, welchen Waldboden, sowie noch nicht haubare Holtbestände für die Waldwirtschaft besitzen. Allgemeine Forst- und Jagdzeitung 20:441-455.

Gadow, K. v. and Puumalainen, J., 2000: Scenario Planning for Sustainable Forest Management. In: Gadow, K. v., Pukkala, T. u. Tomé, M., 2000: Sustainable Forest Management. Kluwer Academic Publisher, Dordrecht: 319-356.

Guldin, J. M., 1996. The role of the uneven-aged silviculture in the context of ecosystem management. Western Journal of Applied Forestry 11(1):4-12.

Hill, L. W., 1992. More on clearcutting. Journal of Forestry 90(8):43.

Hotvedt, J. E. and Ward, K. B., 1990. A dynamic programming optimization model for uneven-aged loblolly-shortleaf pine stands in the mid-South. In: Hickman, C. A.(Ed.), Proc. of the Southern Forest Economics Workshop on Evaluating Even- and All-Aged Timber Management Options for Southern Forest Lands, March 29-30 1990, Monroe, LA. GTR SO-79. USDA For. Serv., Southern Exp. Stn., New Orleans, LA. pp.35-43.

Kluender, R. A., Lortz, D., McCoy, W., Stockes, B. and Klepac, J., 1998. Removal intensity and tree size effects on harvesting costs and profitability. Forest Products Journal 48(1):49-54.

Molina, F., 1988. Los bosques de Galicia. Actualidad Forestal de Galicia. N 106-107 EneroJunio. pp.1-5.

Redmond, C. H., and Greenhalgh, R., 1990. An economic analysis of even-aged versus uneven-aged management on nonindustrial private land in southern Arkansas. In: Hickman, C.A. (Ed.), Proc. of the Southern Forest Economics Workshop on Evaluating Even- and All-Aged Timber Management Options for Southern Forest Lands, March 29-30 1990, Monroe, LA. GTR SO-79. USDA For. Serv., Southern Exp. Stn., New Orleans, LA. pp.69-79.

Rodríguez, R., Álvarez, J. G. and Vega, G., 1993. Revisión de las curvas de calidad de estación de Pinus pinaster en Galicia. Ponencias y Comunicaciones del Congreso Forestal Español. Lourizán. SECF, Xunta de Galicia, Tomo II, pp. 587-592.

Romero, A., 1992. El sector forestal en Galicia. Economía Política Forestal. Xunta de Galicia. Santiago de Compostela. pp. 113-132.

Sánchez, S., and Rodríguez, R., 2002. Modelling the growth and management of unevenaged maritime pine-broadleved species forests in Galicia (NW Spain). Scandinavian Journal of Forest Research (in press). 
Saunders, D. A., Hobbs, R. J. and Margules, C. R., 1991. Biological consequences of ecosystem fragmentation: a review. Conserv. Biol. 5(1):18-32.

Schröder, J., 2000. Analyse der Wuchsdynamik von Pinus pinaster Ait. in Nordwest-Spanien. PhD Thesis, Georg-August-Universität Göttingen. Cuvillier Verlag, Göttingen.

Stokes, B. J., Kluender, R. A, Williams, R. A. and Klepac, J. F., 1993. Assessment of costs and impacts for alternative harvesting methods in mixed stands. In: Brissette, J. C. (Ed), Proc. Of the 7th Biennial Southern Silvicultural Research Conf., November 1719, 1992, Mobile, A. L. GTR SO-93, USDA For. Serv., Southern For. Exp. Stn., New Orleans, LA pp.655-662. 


\section{LEBENSLAUF}

\section{PERSÖNLICHE DATEN}

Name: Sánchez Orois

Vorname: Sofía

Geburtsdatum: 18.11 .76

Geburtsort: Arzúa, Spanien

Familienstand: ledig

Email: ssanche@uni-forst.gwdg.de

Homepage: http://www.stud.uni-goettingen.de/ s135549/Beginn.htm

\section{AKADEMISCHE DATEN}

Seit 01.10.01

$01.10 .00-30.09 .01$

WS 1999/00 - SS 2000

WS 1997/98 - SS 2000

WS 1994/95 - SS 1997

WS 1990/91 - SS 1994

WS 1982/83 - SS 1990
Wissenschaftliche Mitarbeiterin am Institut für Waldinventur und Waldwachstum. Stipendiatin der Stiftung „Caixa Galicia“.

Wissenschaftliche Mitarbeiterin am Institut für Waldinventur und Waldwachstum. Stipendiatin der Stiftung „Barrie de la Maza“. Einjähriges Forschungsstipendium.

ERASMUS Stipendium zur Bearbeitung der Diplomarbeit: „Ein VB6.0 Programm zur Prognose von Pinus pinaster Beständen in Galicia (Spanien)“. Georg-August Universität Göttingen. Note: Sehr gut.

Studium der Forstwissenschaft. Santiago de Compostela Universität. Abschlussnote: Sehr gut.

Technisches Studium der Forstwissenschaft. Vigo Universität.

Besuch des Gymnasiums „A Xunqueira I“. Pontevedra.

Besuch der Grundschule „Colexio Público Pedra Picada“. Lérez. Pontevedra.

\section{BERUFLICHE TÄTIGKEITEN UND PRAKTIKA}

01.02.01-30.09.02 Lehrtätigkeit beim Institut für Waldinventur und Waldwachstum: Betreuung der Lehrveranstaltung „Anwendungen von $M s$-ACCESS und $V B A$ in der Forsteinrichtung" und der Übungen zur Vorlesung „Steuerung und Analyse der Waldentwicklung“.

01.07.98-30.09.98 Praktikum bei „Estación Fitopatológica Do Areeiro.” (Forschungszentrum für Phytopatologie). 